\title{
The metal-poor end of the Spite plateau
}

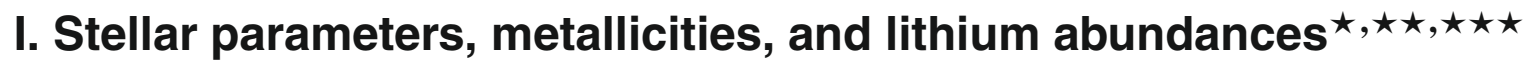

\author{
L. Sbordone ${ }^{1,2,3}$, P. Bonifacio ${ }^{1,2,4}$, E. Caffau ${ }^{2}$, H.-G. Ludwig ${ }^{1,2,5}$, N. T. Behara ${ }^{1,2,6}$, J. I. González Hernández ${ }^{1,2,7}$,

 \\ F. Spite ${ }^{2}$, T. C. Beers ${ }^{12}$, N. Christlieb ${ }^{5}$, P. François ${ }^{2}$, and V. Hill ${ }^{2,13}$
}

${ }^{1}$ CIFIST Marie Curie Excellence Team, France

2 GEPI, Observatoire de Paris, CNRS, Université Paris Diderot, Place Jules Janssen, 92190 Meudon, France

3 Max-Planck Institut für Astrophysik, Karl-Schwarzschild-Str. 1, 85741 Garching, Germany e-mail: lsbordone@mpa-garching.mpg.de

4 INAF - Osservatorio Astronomico di Trieste, via G. B. Tiepolo 11, 34143 Trieste, Italy

5 Zentrum für Astronomie der Universität Heidelberg, Landessternwarte, Königstuhl 12, 69117 Heidelberg, Germany

6 Institut d'Astronomie et d'Astrophysique, Université Libre de Bruxelles, CP 226, boulevard du Triomphe, 1050 Bruxelles, Belgium

7 Dpto. de Astrofísica y Ciencias de la Atmósfera, Facultad de Ciencias Físicas, Universidad Complutense de Madrid, 28040 Madrid, Spain

8 Astrophysikalisches Institut Potsdam An der Sternwarte 16, 14482 Potsdam, Germany

9 Centre de Recherche Astrophysique de Lyon, UMR 5574: Université de Lyon, École Normale Supérieure de Lyon, 46 allée d'Italie, 69364 Lyon Cedex 07, France

10 Université Montpellier 2, CNRS, GRAAL, 34095 Montpellier, France

11 Indian Institute of Astrophysiscs, II Block, Koramangala, Bangalore 560 034, India

12 Dept. if Physics \& Astronomy, and JINA: Joint Insrtitute for Nuclear Astrophysics, Michigan State University, E. Lansing, MI 48824, USA

13 Cassiopée - Observatoire de la Côte d'Azur, Boulevard de l'Observatoire, BP 4229, 06304 Nice Cedex 4, France

Received 11 September 2009 / Accepted 17 March 2010

\section{ABSTRACT}

Context. The primordial nature of the Spite plateau is at odds with the WMAP satellite measurements, implying a primordial Li production at least three times higher than observed. It has also been suggested that $A(\mathrm{Li})$ might exhibit a positive correlation with metallicity below $[\mathrm{Fe} / \mathrm{H}] \sim-2.5$. Previous samples studied comprised few stars below $[\mathrm{Fe} / \mathrm{H}]=-3$.

Aims. We present VLT-UVES Li abundances of 28 halo dwarf stars between $[\mathrm{Fe} / \mathrm{H}]=-2.5$ and -3.5 , ten of which have $[\mathrm{Fe} / \mathrm{H}]<-3$. Methods. We determined stellar parameters and abundances using four different $T_{\text {eff }}$ scales. The direct infrared flux method was applied to infrared photometry. $\mathrm{H} \alpha$ wings were fitted with two synthetic grids computed by means of 1D LTE atmosphere models, assuming two different self-broadening theories. A grid of $\mathrm{H} \alpha$ profiles was finally computed by means of 3D hydrodynamical atmosphere models. The Li I doublet at $670.8 \mathrm{~nm}$ has been used to measure $A(\mathrm{Li})$ by means of 3D hydrodynamical NLTE spectral syntheses. An analytical fit of $A(\mathrm{Li})_{3 \mathrm{D} \text {,NLTE }}$ as a function of equivalent width, $T_{\text {eff }}, \log g$, and $[\mathrm{Fe} / \mathrm{H}]$ has been derived and is made available.

Results. We confirm previous claims that $A(\mathrm{Li})$ does not exhibit a plateau below $[\mathrm{Fe} / \mathrm{H}]=-3$. We detect a strong positive correlation with $[\mathrm{Fe} / \mathrm{H}]$ that is insensitive to the choice of $T_{\text {eff }}$ estimator. From a linear fit, we infer a steep slope of about $0.30 \mathrm{dex}$ in $A(\mathrm{Li})$ per dex in $[\mathrm{Fe} / \mathrm{H}]$, which has a significance of $2-3 \sigma$. The slopes derived using the four $T_{\text {eff }}$ estimators are consistent to within $1 \sigma$. A significant slope is also detected in the $A(\mathrm{Li})-T_{\text {eff }}$ plane, driven mainly by the coolest stars in the sample $\left(T_{\text {eff }}<6250\right)$, which appear to be $\mathrm{Li}$-poor. However, when we remove these stars the slope detected in the $A(\mathrm{Li})-[\mathrm{Fe} / \mathrm{H}]$ plane is not altered significantly. When the full sample is considered, the scatter in $A(\mathrm{Li})$ increases by a factor of 2 towards lower metallicities, while the plateau appears very thin above $[\mathrm{Fe} / \mathrm{H}]=-2.8$. At this metallicity, the plateau lies at $\left\langle A(\mathrm{Li})_{3 \mathrm{D}, \mathrm{NLTE}}\right\rangle=2.199 \pm 0.086$.

Conclusions. The meltdown of the Spite plateau below $[\mathrm{Fe} / \mathrm{H}] \sim-3$ is established, but its cause is unclear. If the primordial $A(\mathrm{Li})$ were that derived from standard BBN, it appears difficult to envision a single depletion phenomenon producing a thin, metallicity independent plateau above $[\mathrm{Fe} / \mathrm{H}]=-2.8$, and a highly scattered, metallicity dependent distribution below. That no star below $[\mathrm{Fe} / \mathrm{H}]=$ -3 lies above the plateau suggests that they formed at plateau level and experienced subsequent depletion.

Key words. nuclear reactions, nucleosynthesis, abundances - Galaxy: halo - Galaxy: abundances - cosmology: observations stars: Population II

^ Based on observations made with the ESO Very Large Telescope at Paranal Observatory, Chile (Programmes 076.A-0463 and 077.D0299).

$\star \star$ Full Table 3 is available in electronic form at the CDS via anonymous ftp to cdsarc.u-strasbg.fr (130.79.128.5) or via http://cdsarc.u-strasbg.fr/viz-bin/qcat?J/A+A/522/A26

$\star \star \star$ IDL code (appendix) is only available in electronic form at http: //www . aanda.org

\section{Introduction}

Spite \& Spite (1982a,b) first noted that metal-poor $(-2.4 \leq$ $[\mathrm{Fe} / \mathrm{H}] \leq-1.4)$, warm $\left(5700 \mathrm{~K} \leq T_{\text {eff }} \leq 6250 \mathrm{~K}\right)$, dwarf stars exhibit a remarkably constant $\mathrm{Li}$ abundance, irrespective of metallicity and effective temperature, and interpreted this plateau in Li abundance (hereafter the Spite plateau) as being representative of the abundance of $\mathrm{Li}$ synthesized during the 
primordial hot and dense phase of the Universe (Big Bang, Wagoner et al. 1967; see Iocco et al. 2009, for a review). Determining the lithium abundance in unevolved metal-poor stars has since developed into an active research topic, because of its potential role as a cosmological diagnostic. In the standard Big Bang nucleosynthesis (SBBN) scenario, ${ }^{7} \mathrm{Li}$ is formed immediately after the Big Bang, together with ${ }^{1} \mathrm{H},{ }^{2} \mathrm{H},{ }^{3} \mathrm{He}$, and ${ }^{4} \mathrm{He} .{ }^{2} \mathrm{H}$ is formed first, and is subsequently required as a seed to form any heavier element (the so-called "deuterium bottleneck"). The abundance of all the subsequent BBN products thus depend on the equilibrium ${ }^{2} \mathrm{H}$ abundance, which is determined by the ${ }^{2} \mathrm{H}$ photodissociation reaction ${ }^{2} \mathrm{H}\left(\gamma,{ }^{1} \mathrm{H}\right){ }^{1} \mathrm{H}$. As a result, all the abundances of BBN products ultimately depend on the primordial baryon/photon ratio $\eta_{\mathrm{B}} \equiv n_{\mathrm{B}} / n_{\gamma}$ (Steigman 2001), and can in principle be employed to constrain this fundamental cosmological parameter.

Following the cosmic microwave background anisotropy measurements of WMAP (e.g. Dunkley et al. 2009), $\eta_{\mathrm{B}}$ can be inferred from the value of the baryonic density, $\Omega_{\mathrm{B}}$, i.e. determining the primordial abundance of $\mathrm{BBN}$ products is no longer the only means by which it is estimated. On the other hand, the comparison between the two estimates remains of paramount importance as a test of the reliability of the BBN theory, of our present understanding of the subsequent chemical evolution of the elements involved, and, in the case of $\mathrm{Li}$, of our understanding of stellar atmospheres.

Among the available BBN products, ${ }^{2} \mathrm{H}$ and ${ }^{7} \mathrm{Li}$ are the most reliable $\eta_{\mathrm{B}}$ indicators. Being ${ }^{2} \mathrm{H}$ never produced in stars, its abundance in a low-metallicity environment can be assumed to be quite close to the cosmological value. In addition, its sensitivity to $\eta_{\mathrm{B}}$ is monotonic and quite strong $\left(\left({ }^{2} \mathrm{H} /{ }^{1} \mathrm{H}\right) \propto \eta_{\mathrm{B}}^{-1.6}\right.$, Steigman 2009). On the other hand, ${ }^{2} \mathrm{H}$ can be effectively measured only in high-redshift, low-metallicity damped Lyman $\alpha$ (DLAs) or Lyman limit systems, for which the observations are so challenging that only seven such high quality measurements exist to date, which were all obtained after $10 \mathrm{~m}$-class telescopes became available (Pettini et al. 2008). The $\eta_{\mathrm{B}}$ value inferred from them is in good agreement with that derived from WMAP (Steigman 2009).

In contrast, ${ }^{7} \mathrm{Li}$ can be measured with relative ease in the photospheres of warm, unevolved stars. The observations are typically restricted to dwarfs, at least when one is interested in determining the primordial $\mathrm{Li}$ abundance, because the fragile Li nucleus is destroyed by the ${ }^{7} \mathrm{Li}(\mathrm{p}, \alpha)^{4} \mathrm{He}$ reaction as soon as the temperature reaches 2.6 million $\mathrm{K}$. This implies that giants should not be considered, since their deep convective zones mix the surface material with layers that exceed this temperature, and almost all Li is rapidly destroyed. The ease with which ${ }^{7} \mathrm{Li}$ is destroyed has always constituted a challenge to existing models of convection and diffusion in stellar atmospheres, which predict a depletion of at least a factor of four relative to the primordial abundance (Michaud et al. 1984). While one could infer that some depletion might have occurred, it appeared impossible to obtain a constant depletion over such a wide range of effective temperatures. The simplest solution was to assume that no depletion was indeed taking place. This is in marked contrast to the solar case, where the photospheric Li abundance is about two dex lower than the meteoritic value.

The original interpretation of the Spite plateau has been challenged in many ways in the years since its discovery. Surely the most compelling challenge was the independent measurement of $\eta_{\mathrm{B}}$ by the WMAP satellite, placing the expected primordial $\mathrm{Li}$ abundance at $A(\mathrm{Li})^{1}{ }_{\mathrm{P}}=2.65_{-0.06}^{+0.05}$ (Steigman 2007), or even higher, $A(\mathrm{Li})_{\mathrm{P}}=2.72 \pm 0.05$ when updated rates are taken into account for the ${ }^{3} \mathrm{He}(\alpha, \gamma)^{7} \mathrm{Li}$ reaction (Cyburt et al. 2008). The highest estimate of the Spite plateau does not exceed $A(\mathrm{Li})=2.4$, a more typical value being $A(\mathrm{Li}) \sim 2.2$. The discrepancy can in principle be eliminated in two ways, by either rejecting the standard BBN scenario (for a review see Iocco et al. 2009), or by assuming that some degree of Li depletion has occurred. Two main mechanisms could again be invoked. Li could be subject to depletion before the currently observed stars are formed (Piau et al. 2006), by means of the reprocessing of the primordial gas in a first generation of massive, hot stars. This phenomenon does not appear to be able to explain the entire WMAP/Spite plateau gap, but, removing up to $0.3 \mathrm{dex}$ of the discrepancy could considerably reduce the problem. The maximum possible depletion is nevertheless dependent on the initial mass function and lifetime of Pop. III stars, as well as on the effectiveness of the mixing of their ejecta in the interstellar medium, which are all poorly known. Alternatively, Li can be depleted within the stars we currently observe, as a consequence of phenomena within the envelope, such as diffusion, gravity waves, rotational mixing, or any combination of these. As stated above, the negligible scatter, and apparent lack of slope in the Spite plateau are observational constraints that models of Li depletion have failed to reproduce. This could apparently be achieved by combining diffusion with some form of turbulence at the bottom of the atmospheric convective zone (Richard et al. 2005; Korn et al. 2006, 2007; Piau 2008; Lind et al. 2009b). Unfortunately, the effect of turbulence is introduced basically as a free parameter, and its tuning is made quite difficult by the subtlety of the effects expected on elements other than $\mathrm{Li}$ (see Sect. 6.4 in Bonifacio et al. 2007). Claims have been made (e.g. Asplund et al. 2006) that the lighter ${ }^{6} \mathrm{Li}$ isotope has been detected in the atmospheres of dwarf stars displaying Spite plateau ${ }^{7} \mathrm{Li}$ abundances. These measurements are very difficult and sensitive to subtle details of the analysis (Cayrel et al. 2007). If the detection of ${ }^{6} \mathrm{Li}$ in EMP dwarf stars were to be confirmed, it would severely undermine any claim of a substantial atmospheric depletion of ${ }^{7} \mathrm{Li}$ during the star's lifetime, since the ${ }^{6} \mathrm{Li}$ is even more easily destroyed than ${ }^{7} \mathrm{Li}$.

One additional problem is constituted by repeated claims that the Spite plateau might display a tilt towards lower Li abundances at lower metallicities, on the order of $0.1-0.2$ dex in $A(\mathrm{Li})$ per dex in [Fe/H] (Ryan et al. 1996, 1999; Boesgaard et al. 2005; Asplund et al. 2006), although other studies failed to confirm this (e.g. Bonifacio \& Molaro 1997). Roughly below $[\mathrm{Fe} / \mathrm{H}]=-2.5$, more and more stars appear to exhibit $\mathrm{Li}$ abundances below the plateau level, while the scatter increases.

The extreme case is possibly represented by the lithium abundance upper limit of the hyper-iron-poor subgiant HE 1327-2326 (Frebel et al. 2008, and references therein), which should have $A(\mathrm{Li}) \leq 0.7$ (from $1 \mathrm{D}$ analysis). The interpretation of this result is not straightforward. Even rejecting the interpretation (Venn \& Lambert 2008) that this star might be a chemically-peculiar evolved object, the unusual photospherical composition of this star has not yet found a satisfactory explanation. Were the composition of HE 1327-2326 to be indeed primordial, its lack of Li would support the Piau et al. (2006) suggestion of a pollution by material cycled through massive Pop. III stars.

Adopting the Piau et al. (2006) hypothesis, one could then envision a scenario in which partial pollution by this astrated

\footnotetext{
$\overline{1} A(\mathrm{Li})=\log [N(\mathrm{Li}) / N(\mathrm{H})]+12$.
} 
material induces varying degrees of Li "depletion" in EMP stars according to how much this reprocessed gas is available locally at the location and time of each stars' formation. A linear fit to EMP stellar Li abundances would then naturally lead to an expected trend in $A(\mathrm{Li})$ with $[\mathrm{Fe} / \mathrm{H}]$, whose slope would appear steeper the more the sample is limited to low metallicities. An alternative explanation would be to postulate that a $\mathrm{Li}$ "overdepletion" mechanism operates in the photospheres of the most metal-poor stars, a mechanism that would not act uniformly in every star of a given metallicity (possibly depending on $T_{\text {eff }}$ or rotation speed or both), but would be more efficient at lower $[\mathrm{Fe} / \mathrm{H}]$. These stars would then begin with a $\mathrm{Li}$ abundance corresponding to the Spite plateau, but most of them would then develop some degree of Li depletion. This explanation would, at the same time, explain the apparent slope at low metallicities and the increase in the scatter. It would also explain why, even at very low metallicity, one still finds some stars lying on the Spite plateau. A striking example of this is the EMP double-lined binary system CS 22876-032 (González Hernández et al. 2008), in which, at $[\mathrm{Fe} / \mathrm{H}]=-3.6$, the primary lies on the Spite plateau, while the secondary has a $\mathrm{Li}$ abundance lower by about 0.4 dex.

\section{Observations and data reduction}

Our sample includes 11 main-sequence turnoff and dwarf stars selected from various sources (see Table 2), along with the sample already presented in Bonifacio et al. (2007). The star HE 0148-2611 was previously analyzed (Cohen et al. 2002; Carretta et al. 2002) but no Li measurement was ever performed. One star (HE 1413-1954) was derived from the Barklem et al. (2005) sample. It, again, had no previous Li measurement. The remaining targets were drawn from the HK (Beers et al. 1985, 1992; Beers 1999) and Hamburg/ESO (Christlieb et al. 2008) surveys, and were never studied previously based on high-resolution spectra. They were observed by VLT-UVES (Dekker et al. 2000) during programmes 076.A-0463(A) (P. I. Lopez, HE 1413-1954 and BS 17572-0100) and 077.D-0299(A) (P. I. Bonifacio, remaining targets). The observation log for the 11 new targets is in Table 1, where the final signal-to-noise ratio $(S / N)$ around the Li I $670.8 \mathrm{~nm}$ doublet is also indicated. For the two stars observed during 076.A-0463(A), the observations were performed by using VLT-UVES with the DIC1 dichroic and the $346 \mathrm{~nm}+580 \mathrm{~nm}$ setting with a $1^{\prime \prime}$. 0 slit. These observations thus do not contain the 380-480 nm range, but for HE 1413-1954 the HERES (Barklem et al. 2005) data were available, which covered that wavelength range. For the stars observed during 077.D0299(A), we used DIC1 with the $390 \mathrm{~nm}+580 \mathrm{~nm}$ setting and 1 .'0 slit, thus providing coverage from $360 \mathrm{~nm}$ to $750 \mathrm{~nm}$. All the spectra have spectral resolution of $R \sim 40000$. The data were reduced using the standard UVES pipeline. In Fig. 1, we show the Li I $670.8 \mathrm{~nm}$ line region for the 11 newly observed stars.

The data were reduced and analyzed with the same procedures used in Bonifacio et al. (2007), to which the interested reader is referred for details of the analysis and the associated uncertainties. An extract of the table listing the employed Fe I and $\mathrm{Fe}$ II lines, in addition to associated atomic data, equivalent widths, and abundances in the 3D scale is available in Table 3. The full table is available at the CDS.

\section{Atmosphere models and spectrosynthesis programs}

\subsection{D LTE models and spectrosynthesis}

Various one-dimensional (1D) local thermodynamical equilibrium (LTE) atmosphere models were employed in the present
Table 1. Observations $\log$ for the 11 new targets.

\begin{tabular}{|c|c|c|c|c|c|}
\hline Star & $\begin{array}{l}\text { Obs. date } \\
\text { (UT) }\end{array}$ & $\begin{array}{l}\mathrm{MJD}^{a} \\
(\mathrm{UT})\end{array}$ & $\begin{array}{l}\text { Exp. time } \\
\text { s }\end{array}$ & $\begin{array}{c}V_{\mathrm{rad}^{b}} \\
\mathrm{~km} \mathrm{~s}^{-1}\end{array}$ & $S / N^{c}$ \\
\hline BS 17572-100 & 21 Feb. 2006 & 53787.05628641 & 3190 & 189 & 191 \\
\hline CS 22188-033 & 7 Jul. 2006 & 53923.31274319 & 3035 & 14 & 107 \\
\hline CS 22882-027 & 6 Jul. 2006 & $\begin{array}{l}53922.31576391 \\
53922.36486180\end{array}$ & $\begin{array}{l}4100 \\
4100\end{array}$ & $\begin{array}{l}182 \\
182\end{array}$ & 78 \\
\hline CS 22950-173 & 23 Apr. 2006 & 53848.33688352 & 3600 & 69 & 92 \\
\hline CS 29491-084 & 17 May 2006 & 53872.31418739 & 3600 & -8 & 104 \\
\hline CS 29514-007 & 7 Jul. 2006 & 53923.35103355 & 3600 & 41 & 91 \\
\hline CS 29516-028 & 19 May 2006 & $\begin{array}{l}53874.32992778 \\
53874.37328577\end{array}$ & $\begin{array}{l}3600 \\
3600\end{array}$ & $\begin{array}{l}-179 \\
-179\end{array}$ & 62 \\
\hline CS 30302-145 & 23 Apr. 2006 & $\begin{array}{l}53848.28216715 \\
53848.30823918\end{array}$ & $\begin{array}{l}2160 \\
2160\end{array}$ & $\begin{array}{l}195 \\
195\end{array}$ & 70 \\
\hline CS 30344-070 & $\begin{array}{l}17 \text { May } 2006 \\
27 \text { May } 2006\end{array}$ & $\begin{array}{l}53872.35925533 \\
53882.31201609\end{array}$ & $\begin{array}{l}2160 \\
2160\end{array}$ & $\begin{array}{l}-141 \\
-140\end{array}$ & 82 \\
\hline HE 0148-2611 & 12 Jul. 2006 & 53928.37446515 & 3600 & -227 & 72 \\
\hline HE 1413-1954 & 21 Feb. 2006 & 53787.27197427 & 3600 & -101 & 52 \\
\hline
\end{tabular}

Notes. ${ }^{(a)}$ Modified Julian date of observation start: MJD = JD$2400000.5{ }^{(b)}$ Rounded to the nearest $\mathrm{km} \mathrm{s}^{-1}$, barycentric correction applied. ${ }^{(c)}$ Near Li I $670.8 \mathrm{~nm}$ doublet, if more than one spectrum has been used, $S / N$ is measured in the coadded spectrum.

study. Castelli's grid of fluxes computed using ATLAS 9 (Castelli \& Kurucz 2003) ${ }^{2}$ was used in the infrared flux temperature determination (see Sect. 4.2). A second ATLAS 9 model grid (Kurucz 2005; Sbordone et al. 2004; Sbordone 2005) was computed with an ad hoc mixing length parameter in producing the $\mathrm{H} \alpha$-wing profiles used to determine $T_{\text {eff }}$ (see Sect. 4.1). It has been shown (Fuhrmann et al. 1993; van't Veer-Menneret \& Megessier 1996) that employing in ATLAS a mixing length parameter of $l / H_{\mathrm{p}}=0.5$ provides the best fit to Balmer lines profiles in the Sun, while the value $l / H_{\mathrm{p}}=1.25$ generally better reproduces more closely the solar flux, and thus, is usually employed in "general purpose" models. To compute these profiles, we employed a modified version of Kurucz's code BALMER $^{3}$, which was capable of handling different linebroadening theories. Finally, we employed OSMARCS atmosphere models (Gustafsson et al. 1975; Plez et al. 1992; Edvardsson et al. 1993; Asplund et al. 1997; Gustafsson et al. 2003) and the turbospectrum spectral synthesis code (Alvarez \& Plez 1998) to determine Fe I and Fe II abundances, gravity, microturbulence, and Li abundances. Hydrostatic monodimensional LHD models (see Caffau \& Ludwig 2007; Caffau et al. 2007) were used in determining the 1D NLTE corrections (see Sect. 6.1).

\subsection{D hydrodynamical models and spectrosynthesis}

Time-dependent, hydrodynamical 3D stellar atmosphere models computed with $\mathrm{CO}^{5}$ BOLD (Freytag et al. 2002; Wedemeyer et al. 2004) as part of the CIFIST model grid (Ludwig et al. 2009b) ${ }^{4}$

\footnotetext{
${ }^{2}$ Grid available at

http://wwwuser.oat.ts.astro.it/castelli/

3 The original version is available online at

http://kurucz . harvard.edu/

4 See http://cifist.obspm.fr/
} 


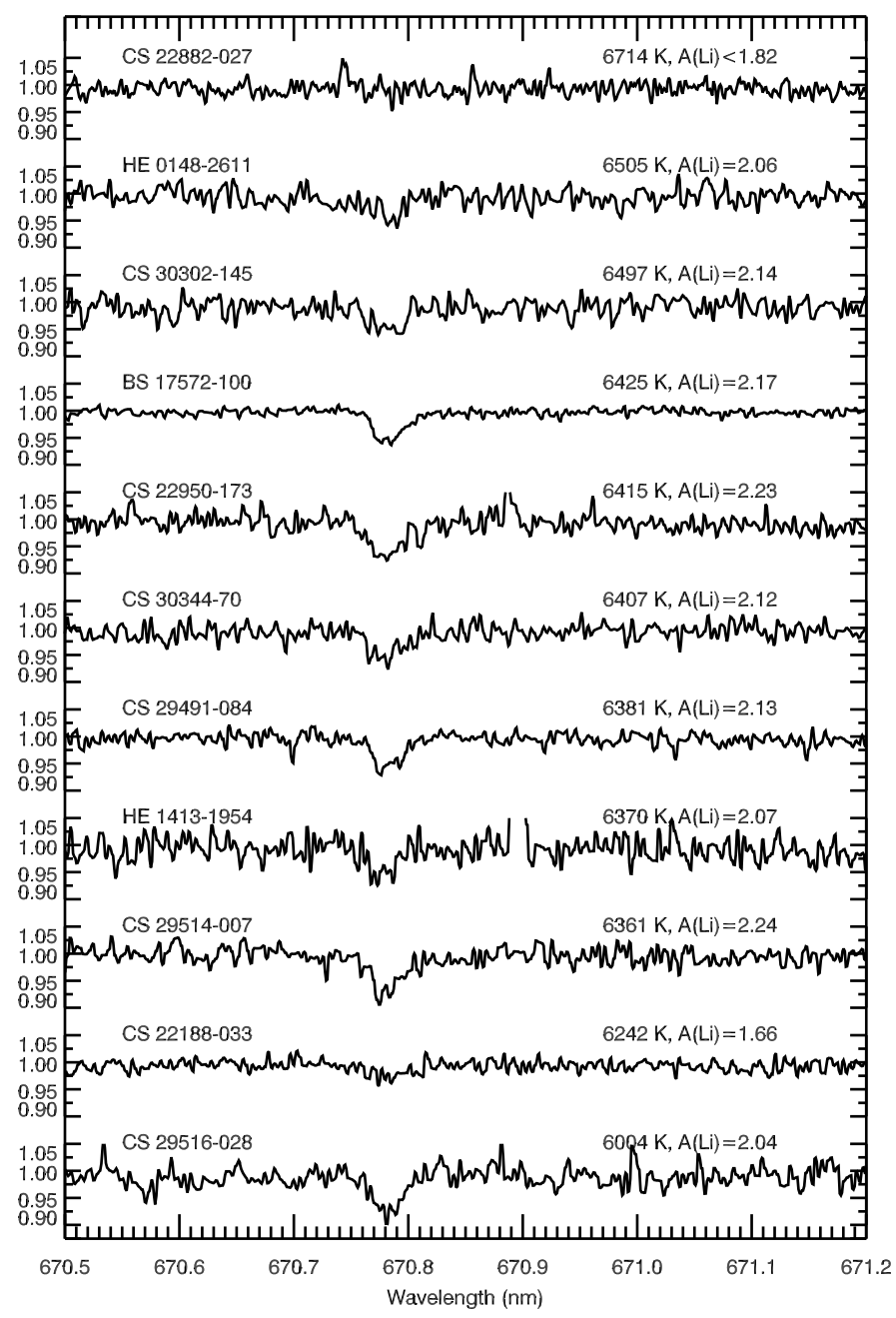

Fig. 1. High-resolution spectra of the Li I $670.79 \mathrm{~nm}$ doublet region for the 11 newly observed stars of the sample. For stars for which multiple spectra were available, the coadded spectrum is shown. For the purpose of visualization, all the spectra have been shifted to zero radial velocity and normalized. $T_{\text {eff }}$ increases from bottom to top, 3D scale $T_{\text {eff }}$ and $A(\mathrm{Li})_{3 \mathrm{D}, \mathrm{NLTE}}$ (see Sects. 4 and 6) are listed for each star. The star CS 22882-027 shows no detectable Li line, and the $3 \sigma$ upper limit to $A(\mathrm{Li})$ is listed here.

were employed to produce grids of $\mathrm{H} \alpha$-wing profiles for $T_{\text {eff }}$ estimation (see Sect. 4.1; and Ludwig et al. 2009a; Behara et al. 2009).

\section{Effective temperature}

Effective temperature ( $\left.T_{\text {eff }}\right)$ is the most crucial stellar atmosphere parameter influencing $\mathrm{Li}$ abundance determination, $\mathrm{Li}$ abundances derived from the Li I $670.75 \mathrm{~nm}$ line being sensitive to $T_{\text {eff }}$ at the level of about 0.03 dex for each $50 \mathrm{~K}$ variation in $T_{\text {eff. }}$. Unfortunately, a precise determination of stellar effective temperatures is generally difficult to achieve. For F/G dwarf and subgiant stars such as those studied here, $T_{\text {eff }}$ is routinely estimated either from photometric calibrations (e.g., Alonso et al. 2000, 2001) or by fitting the wings of $\mathrm{H} \alpha$ with a grid of synthetic profiles of varying $T_{\text {eff }}$.

Both methods are plagued by specific accuracy issues. Photometric calibrations, or the infrared flux method (IRFM), are mainly sensitive to the accuracy of the photometry available, to the details of the calibration process, and to uncertainties in the interstellar reddening estimates. On the other hand, $\mathrm{H} \alpha$ fitting is mainly sensitive to both the uncertainty in the continuum normalization across the broad line wings, and the choice of the broadening theory applied in the line synthesis (see Sect. 4.1).

In this paper, we considered four temperature estimators:

- temperatures derived from $\mathrm{H} \alpha$-wing fitting, using 1D atmosphere models and spectrosynthesis, self broadening being treated according to Barklem et al. (2000a,b) and Stark broadening according to Stehlé \& Hutcheon (1999) (we will henceforth refer to these temperatures as "BA temperatures", or the "BA temperature scale");

- same as BA, but using the Ali \& Griem (1966) selfbroadening theory (ALI temperatures);

- temperatures derived from $\mathrm{H} \alpha$-wing fitting, using 3D model atmospheres and spectrosynthesis, Barklem et al. (2000a,b) self broadening and Stehlé \& Hutcheon (1999) Stark broadening (3D temperatures, for all $\mathrm{H} \alpha$ derived temperatures see Sect. 4.1);

- temperature derived with the infrared flux method (see Sect. 4.2; as well as González Hernández \& Bonifacio 2009, IRFM temperatures).

\subsection{Fitting of the $\mathrm{H} \alpha$ wings}

Temperature scales based on $\mathrm{H} \alpha$-wing fitting are affected by both observational and theoretical issues. Most high-resolution spectrographs use echelle gratings operating in high orders, which exhibit a steep blaze function. The continuum placement is thus sensitive to the accuracy with which the shape of the grating blaze function can be estimated. Such uncertainties are irrelevant when studying narrow lines observed at high resolution, but are important when a broad feature such as $\mathrm{H} \alpha$ is considered. More generally, the precision of continuum placement and of the determination of the $\mathrm{H} \alpha$ wing shape are affected by noise as well as by the possible presence of weak unrecognized features (less of a problem for metal-poor stars). Among these, the blaze function shape likely introduces the largest uncertainty.

On the theoretical side, the uncertainties are due both to the atmosphere model structure and to the physics employed in the $\mathrm{H} \alpha$ synthesis. $\mathrm{H} \alpha$-wing self broadening can be treated with different theories, most notably those of Ali \& Griem (1966), Barklem et al. (2000a,b), and Allard et al. (2008). As a general rule, Ali \& Griem (1966) theory leads to a significantly lower broadening coefficient with respect to the ones derived from Barklem et al. (2000a,b) and Allard et al. (2008). A significantly higher $T_{\text {eff }}$ is required to reproduce a given observed profile when employing the Ali \& Griem (1966) theory with respect to the other theories. With the typical parameters of the stars in our sample, and using our fitting procedure, employing Ali \& Griem (1966) self broadening leads to derived $T_{\text {eff }}$ estimates that are higher by about 150-200 K (a difference of about 0.1 dex in $\mathrm{Li}$ abundance) with respect to those derived by using the Barklem et al. (2000a,b) theory. The theory by Allard et al. (2008), on the other hand, leads to $T_{\text {eff }}$ within a few tens of $\mathrm{K}$ of the $T_{\text {eff }}$ estimates obtained when using the Barklem et al. (2000a,b) theory. We thus restricted ourselves to using the selfbroadening theory of Barklem et al. (2000a,b) (in BA and 3D temperatures) and the Ali \& Griem (1966) self-broadening theory (ALI temperatures).

The $\mathrm{H} \alpha$-fitting temperatures exhibit a significant gravity sensitivity. Barklem et al. (2002) already reported estimates of this sensitivity for relatively metal-poor models (down to $[\mathrm{Fe} / \mathrm{H}]=$ -2 ). The effect is always in the sense of higher gravity leading to 
L. Sbordone et al.: The metal-poor end of the Spite plateau. I.

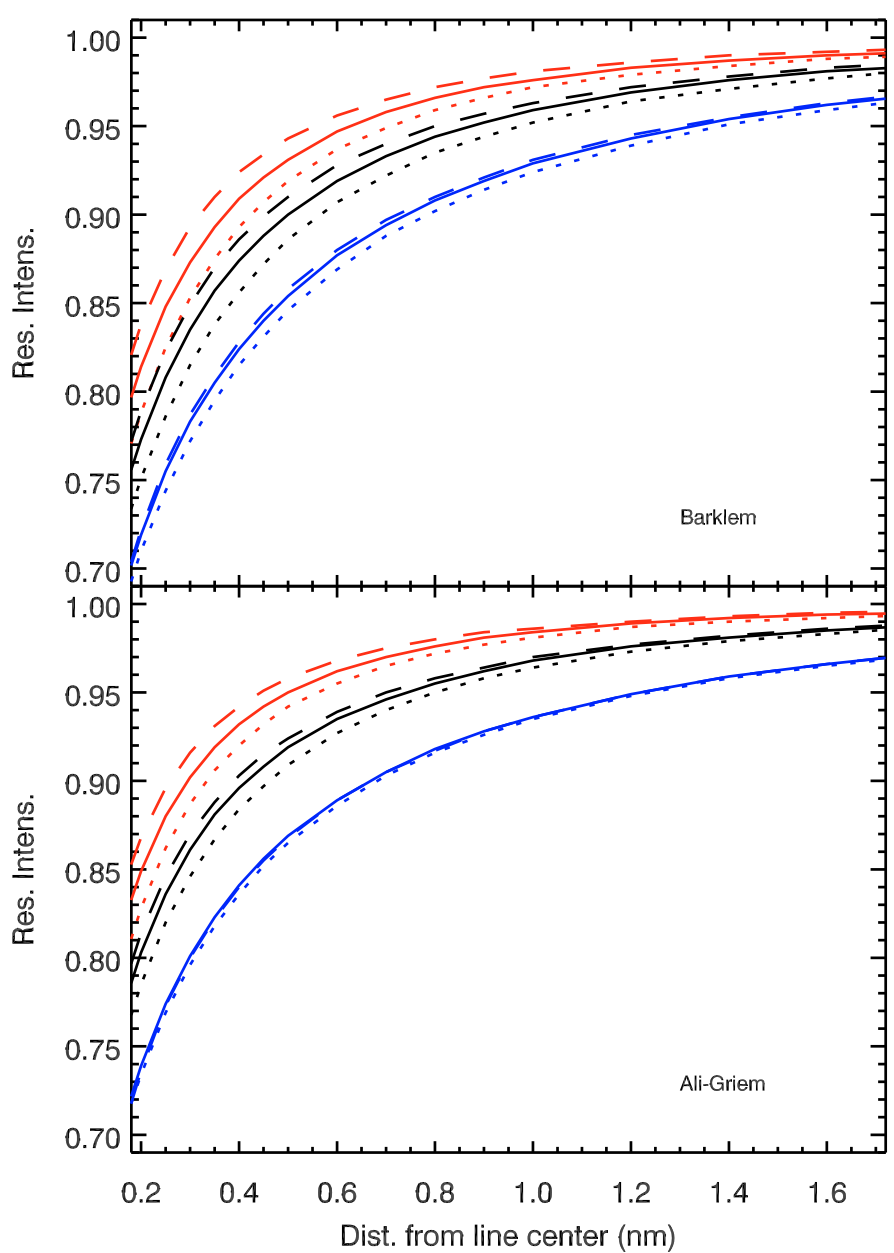

Fig. 2. $\mathrm{H} \alpha$ red wing profiles in the wavelength range significant for the fit. In each panel, red profiles (upper ones) are for $T_{\text {eff }}=5400 \mathrm{~K}$, black profiles (middle ones) are for $T_{\mathrm{eff}}=6000 \mathrm{~K}$, and blue profiles (lower) for $T_{\text {eff }}=6600 \mathrm{~K}$. For each temperature, dashed profiles are for $\log g=$ 3.5, solid profiles for $\log g=4.0$, and dotted profiles are for $\log g=4.5$. All profiles assume $[\mathrm{Fe} / \mathrm{H}]=-3$. Upper panel shows profiles for BA temperatures, lower panel for ALI temperatures.

broader profiles, and appears generally stronger at lower metallicities, at lower temperatures, and for the BA profiles compared to the ALI profiles. In Fig. 2, we plot examples of profiles for the ALI and BA cases. Profiles are plotted for $T_{\text {eff }}=5400,6000$, and $6600 \mathrm{~K}$ (higher temperatures generate broader profiles). A metallicity of $[\mathrm{Fe} / \mathrm{H}]=-3$ is used. For each temperature, we plot the profile for $\log g=3.5,4$, and 4.5. As can be noted, the part of the wing closest to the core appears to be more strongly affected than other parts. It is clearly seen that the gravity sensitivity of the BA profiles is roughly twice as large as in the ALI case. In both the BA and ALI scales, the gravity effect becomes quickly negligible as $T_{\text {eff }}$ increases above $6500 \mathrm{~K}$. In the most deviant cases $\left([\mathrm{Fe} / \mathrm{H}]<-3, T_{\text {eff }}<6000 \mathrm{~K}, \mathrm{BA}\right.$ profiles), a difference of 0.5 dex in $\log g$ leads to roughly a $200 \mathrm{~K}$ difference in $T_{\text {eff }}$.

Thus, the shape of the $\mathrm{H} \alpha$ profile varies in different ways when varying gravity and temperature. As a consequence, the use of an incorrect value of gravity will always affect the temperature estimate, but the size of the effect will depend on the details of how the actual fitting is performed. To provide some insight into what the effect is when employing our specific fitting procedure, we fed the fitting program with $\log g=4$, and $[\mathrm{Fe} / \mathrm{H}]=$ -3 and -2.5 theoretical $\mathrm{H} \alpha$ profiles, and derived the temperature

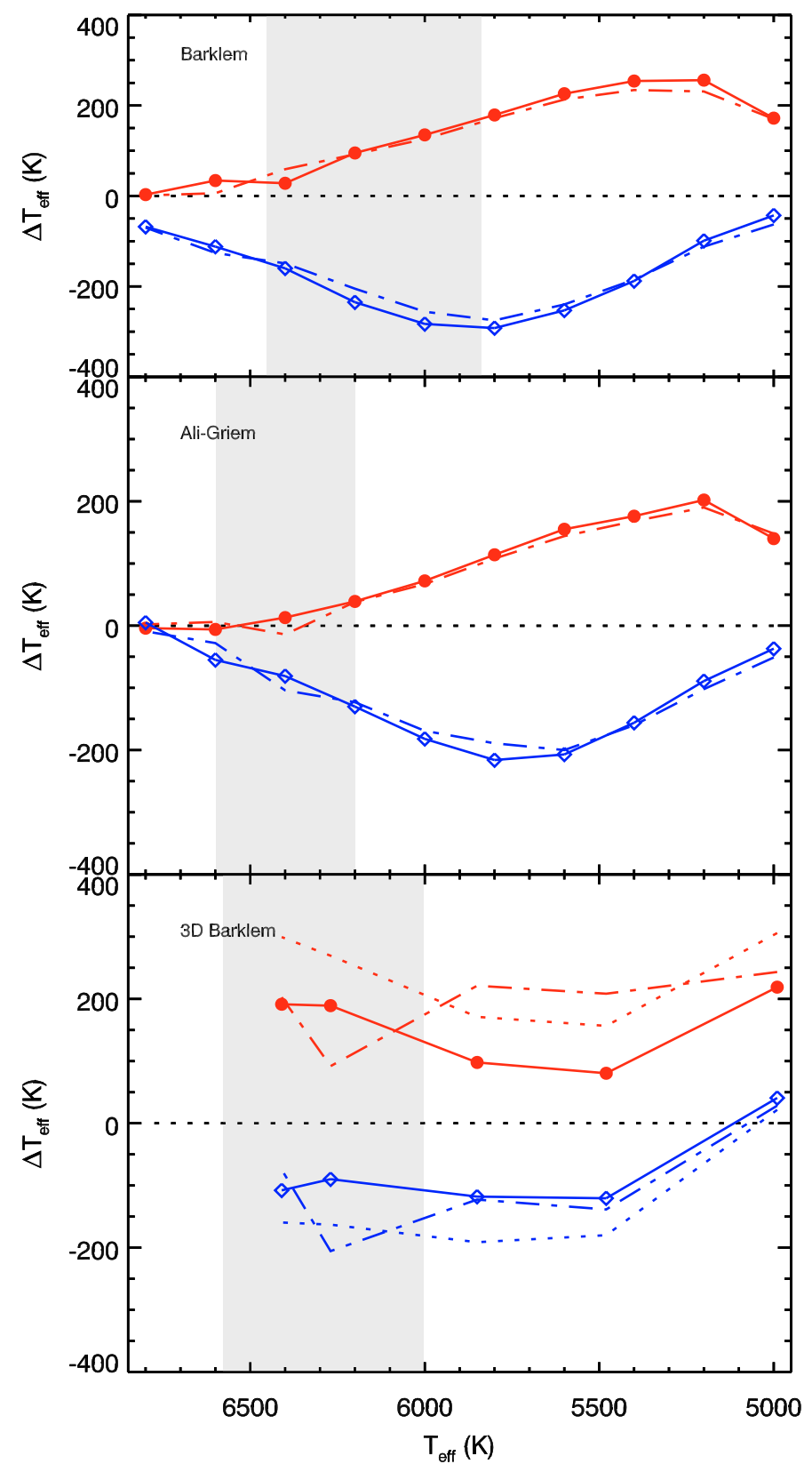

Fig. 3. $\mathrm{H} \alpha$ theoretical profiles for models with $\log g=4,[\mathrm{Fe} / \mathrm{H}]=-3$ (solid lines) and $\log g=4,[\mathrm{Fe} / \mathrm{H}]=-2.5$ (dot-dashed lines) have been fitted with the same procedure used for the program stars, but using a $\log g=3.5$ grid (red lines with filled circles) and a $\log g=4.5$ grid (blue lines with open diamonds). Here we plot the temperature difference $\left(T_{\text {eff }}(\log g=3.5 / 4.5)-T_{\text {eff }}(\log g=4.0)\right)$, against the "real" effective temperature of the profile. The upper panel shows BA profiles, the middle panel ALI profiles, and lower panel 3D profiles. The gray shaded areas indicates the temperature ranges for the program stars in each $T_{\text {eff }}$ scale.

by assuming that $\log g=3.5,4.0$, and 4.5. When fitting profiles of $\log g=4.0$ by means of profiles of $\log g=3.5$, which are narrower at each temperature, we obtain a higher temperature estimate than we would if we were to use the proper gravity. The opposite effect occurs when using the broader $\log g=4.5$ profiles. In Fig. 3, we plot these temperature differences versus the true $T_{\text {eff }}$ of the profiles. Differences are computed in the sense $\Delta T_{\text {eff }}=T_{\text {eff }}(\log g=3.5$ or 4.5$)-T_{\text {eff }}(\log g=4.0)$. Red lines with filled circles correspond to fits with $\log g=3.5$ profiles, blue 
ones with open diamonds to fits with $\log g=4.5$ profiles. The solid lines correspond to $[\mathrm{Fe} / \mathrm{H}]=-3.0$ profiles (both fitted and fitting), while the dot-dashed line corresponds to $[\mathrm{Fe} / \mathrm{H}]=-2.5$. For the parameter space covered, and when adopting our fitting procedure, underestimating the fitting-grid gravity by 0.5 dex leads to an overestimate of the temperature by as much as $250 \mathrm{~K}$ in the BA case, and $200 \mathrm{~K}$ in the ALI case. This underestimate reaches a maximum around $5200-5300 \mathrm{~K}$, decreasing on both sides, and fading away on the hot side, near $T_{\text {eff }}=6500 \mathrm{~K}$. By overestimating the fitting-grid gravity, one underestimates $T_{\mathrm{eff}}$ by as much as $300 \mathrm{~K}$ in the BA case and $200 \mathrm{~K}$ in the ALI case. The shape of the curve is similar, but the point of maximum sensitivity occurs between 5800 and $6000 \mathrm{~K}$. There is a hint that the effect decreases mildly at $[\mathrm{Fe} / \mathrm{H}]=-2.5$, although higher metallicities have not been explored.

Since we estimate surface gravity from the Fe I-Fe II ionization equilibrium, the derived gravity is temperature sensitive, so that the two estimations need to be iterated to convergence. As a general rule, we stopped iterating when $T_{\text {eff }}$ variations became lower than $50 \mathrm{~K}$, which typically required not more than 3 iterations, starting from an initial guess of $\log g=4$.

A very mild metallicity sensitivity is also present in the $\mathrm{H} \alpha$ based temperature determination, never surpassing some tens of $\mathrm{K}$ for a $0.5 \mathrm{dex}$ of variation in $[\mathrm{Fe} / \mathrm{H}]$. The actual iteration of the temperature determination with the other atmosphere parameters was performed differently for the BA and ALI cases on one side, and for the $3 \mathrm{D}$ case on the other side:

- In the BA and ALI case, once the gravity and metallicity were determined with one temperature estimate, the $\mathrm{H} \alpha$ profile grid was interpolated to that gravity value, while the nearest grid step was chosen in metallicity, without interpolation. The small metallicity step of the grid $(0.25 \mathrm{dex})$, as well as the very mild sensitivity of $\mathrm{H} \alpha$ to metallicity, made this choice sufficiently precise.

- In the 3D case, the computation of both model atmospheres and spectral synthesis is very time consuming, and only the atmosphere model grids for $\log g=4$ and $\log g=4.5$ with $[\mathrm{Fe} / \mathrm{H}]=-3.0$ were sufficiently extended at the time of the analysis. We thus fitted the observed $\mathrm{H} \alpha$ lines to these two grids, deriving, for each star, effective temperatures corresponding to the two assumed gravities. We determined gravity and metallicity, then derived a new $T_{\text {eff }}$ estimate by linearly interpolating between the $T_{\text {eff }}(\log g=4.0)$ and $T_{\text {eff }}(\log g=4.5)$ at the estimated gravity. The procedure was then iterated but, for most stars, the same convergence criterion applied to the $1 \mathrm{D}$ case $(\Delta T<50 \mathrm{~K})$ was found to be too stringent, since the parameters for most stars ended up oscillating between two sets corresponding to $T_{\text {eff }}$ estimates that were about $60 \mathrm{~K}$ apart. This can probably be attributed to the use of a more coarse grid in the $3 \mathrm{D}$ case.

\subsection{IRFM temperature estimation}

Originally introduced by Blackwell \& Shallis (1977), and later improved by Blackwell et al. (1980), who removed an unnecessary iteration (see Blackwell et al. 1990, and references therein), the infrared flux method (IRFM) relies on the ratio of the flux at a near-infrared (NIR) wavelength or in a NIR band, to the bolometric flux. This ratio can also be derived from model atmospheres, and the effective temperature determined by finding the effective temperature of the model that reproduces the observed ratio. González Hernández \& Bonifacio (2009) presented a new implementation of the method making use of $2 \mathrm{MASS}$

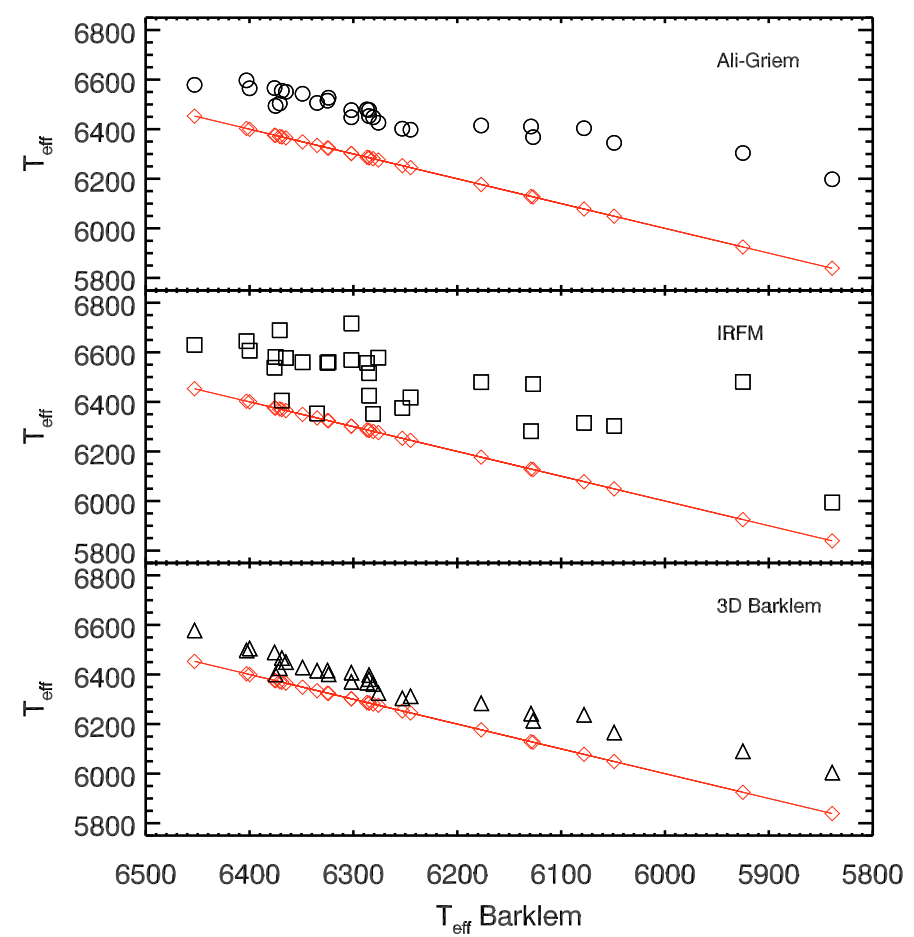

Fig. 4. Effective temperatures for different estimators, plotted against BA temperature for the program stars. Top to bottom: ALI, IRFM and $3 \mathrm{D}$ temperatures. The red line represents the one-to-one relation (hence the line of BA temperatures).

photometry (Skrutskie et al. 2006), and also provided a calibration of bolometric fluxes with colors $(V-J),(V-H)$, and $\left(V-K_{\mathrm{s}}\right)$, where $V$ is in the Johnson system and the NIR magnitudes are in the 2MASS system. We applied the IRFM in exactly the way described by González Hernández \& Bonifacio (2009), deriving the bolometric fluxes as the average of those estimated from the three visible-NIR colors. All magnitudes and colors used in the IRFM must be corrected for reddening. To do so, we used the reddening maps of Schlegel et al. (1998), corrected as described in Bonifacio et al. (2000). All our program stars are sufficiently distant that they lie outside the dust layer, so that the full reddening derived from the maps should be applied. The adopted reddenings are provided in Table 2. The star CS 22882-027 does not appear in the 2MASS catalog, thus we could not derive its IRFM temperature.

\section{Gravity, microturbulence and metallicity}

The FITLINE code was employed to measure the equivalent widths of the Fe I and Fe II lines. Although up to $\sim 120 \mathrm{Fe}$ I lines were available, only four Fe II lines were strong enough to be used. For each temperature scale, gravity was then derived by enforcing $\mathrm{Fe}$ I-Fe II ionization equilibrium. For the $\mathrm{H} \alpha$-based scales, gravity was used with metallicity (Fe I abundance) to iterate the $T_{\text {eff }}$ estimation (see Sect. 4.1).

For each temeperature scale, microturbulence was determined by ensuring that the weak and strong Fe I lines provide the same abundance. The final parameters and the derived metallicity for each temperature estimator are presented in Table 4, detailed Fe I and Fe II abundances are listed in Table 5. Final $T_{\text {eff }}$ values for the ALI, IRFM, and 3D temperature scale are plotted against the BA scale in Fig. 4, and against the respective value of $[\mathrm{Fe} / \mathrm{H}]$ in Fig. 5. 
L. Sbordone et al.: The metal-poor end of the Spite plateau. I.

Table 2. Coordinates and optical and infrared photometry for the program stars.

\begin{tabular}{|c|c|c|c|c|c|c|c|}
\hline Star & $\alpha$ & $\delta$ & V & $J$ & $H$ & $K$ & $E(B-V)$ \\
\hline BS 16023-046 & $14^{\mathrm{h}} 00^{\mathrm{m}} 54^{\mathrm{s}} .6$ & $+22^{\circ} 46^{\prime} 48^{\prime \prime}$ & 14.17 & 13.24 & 13.02 & 12.96 & 0.01801 \\
\hline BS $17570-063$ & $00^{\mathrm{h}} 20^{\mathrm{m}} 36^{\mathrm{s}} .1$ & $+23^{\circ} 47^{\prime} 38^{\prime \prime}$ & 14.51 & 13.47 & 13.17 & 13.07 & 0.03949 \\
\hline BS $17572-100$ & $09^{\mathrm{h}} 28^{\mathrm{m}} 55^{\mathrm{s}} \cdot 3$ & $-05^{\circ} 21^{\prime} 36^{\prime \prime}$ & 12.17 & 11.28 & 11.02 & 10.95 & 0.03727 \\
\hline CS 22177-009 & $04^{\mathrm{h}} 07^{\mathrm{m}} 40^{\varsigma} .5$ & $-25^{\circ} 02^{\prime} 40^{\prime \prime}$ & 14.27 & 13.25 & 12.96 & 13.03 & 0.04407 \\
\hline CS 22188-033 & $00^{\mathrm{h}} 51^{\mathrm{m}} 25^{\mathrm{s}} .9$ & $-38^{\circ} 12^{\prime} 18^{\prime \prime}$ & 13.20 & 12.16 & 11.91 & 11.90 & 0.01315 \\
\hline CS 22882-027 & $00^{\mathrm{h}} 38^{\mathrm{m}} 09.7$ & $-31^{\circ} 47^{\prime} 54^{\prime \prime}$ & 15.11 & - & - & - & - \\
\hline CS 22888-031 & $23^{\mathrm{h}} 11^{\mathrm{m}} 32^{\mathrm{s}} .4$ & $-35^{\circ} 26^{\prime} 43^{\prime \prime}$ & 14.90 & 13.91 & 13.75 & 13.65 & 0.01417 \\
\hline CS 22948-093 & $21^{\mathrm{h}} 50^{\mathrm{m}} 31^{\mathrm{s}} .5$ & $-41^{\circ} 07^{\prime} 49^{\prime \prime}$ & 15.18 & 14.29 & 13.98 & 14.00 & 0.01576 \\
\hline CS 22950-173 & $20^{\mathrm{h}} 35^{\mathrm{m}} 31^{\mathrm{s}} .2$ & $-15^{\circ} 53^{\prime} 30^{\prime \prime}$ & 14.04 & 12.98 & 12.70 & 12.66 & 0.04551 \\
\hline CS 22953-037 & $01^{\mathrm{h}} 25^{\mathrm{m}} 06^{\mathrm{s}} .8$ & $-59^{\circ} 15^{\prime} 58^{\prime \prime}$ & 13.64 & 12.68 & 12.44 & 12.46 & 0.02796 \\
\hline CS 22965-054 & $22^{\mathrm{h}} 06^{\mathrm{m}} 30^{\mathrm{s}} .0$ & $-02^{\circ} 32^{\prime} 39^{\prime \prime}$ & 15.10 & 13.86 & 13.58 & 13.45 & 0.13321 \\
\hline CS 22966-011 & $23^{\mathrm{h}} 35^{\mathrm{m}} 06^{\mathrm{s}} .6$ & $-30^{\circ} 22^{\prime} 53^{\prime \prime}$ & 14.55 & 13.54 & 13.23 & 13.27 & 0.01391 \\
\hline CS 29491-084 & $22^{\mathrm{h}} 28^{\mathrm{m}} 49^{\mathrm{s}} .5$ & $-28^{\circ} 57^{\prime} 03^{\prime \prime}$ & 13.48 & 12.52 & 12.25 & 12.20 & 0.01367 \\
\hline CS 29499-060 & $23^{\mathrm{h}} 53^{\mathrm{m}} 40^{\mathrm{s}} \cdot 2$ & $-26^{\circ} 58^{\prime} 44^{\prime \prime}$ & 13.03 & 12.10 & 11.85 & 11.86 & 0.02027 \\
\hline CS 29506-007 & $21^{\mathrm{h}} 20^{\mathrm{m}} 28^{\mathrm{s}} \cdot 6$ & $-20^{\circ} 46^{\prime} 24^{\prime \prime}$ & 14.18 & 13.17 & 12.93 & 12.87 & 0.04547 \\
\hline CS 29506-090 & $21^{\mathrm{h}} 30^{\mathrm{m}} 28^{\mathrm{s}} .9$ & $-22^{\circ} 10^{\prime} 41^{\prime \prime}$ & 14.33 & 13.34 & 13.10 & 13.07 & 0.04547 \\
\hline CS 29514-007 & $01^{\mathrm{h}} 06^{\mathrm{m}} 40.6$ & $-24^{\circ} 58^{\prime} 41^{\prime \prime}$ & 13.97 & 12.96 & 12.67 & 12.66 & 0.02375 \\
\hline CS 29516-028 & $22^{\mathrm{h}} 25^{\mathrm{m}} 40^{\mathrm{s}} \cdot 3$ & $+05^{\circ} 37^{\prime} 40^{\prime \prime}$ & 15.02 & 13.63 & 13.29 & 13.15 & 0.12816 \\
\hline CS 29518-020 & $01^{\mathrm{h}} 12^{\mathrm{m}} 12^{\mathrm{s}} .9$ & $-31^{\circ} 00^{\prime} 06^{\prime \prime}$ & 14.00 & 13.06 & 12.76 & 12.74 & 0.02241 \\
\hline CS 29518-043 & $01^{\mathrm{h}} 18^{\mathrm{m}} 38^{\mathrm{s}} .2$ & $-30^{\circ} 41^{\prime} 02^{\prime \prime}$ & 14.57 & 13.64 & 13.35 & 13.37 & 0.02030 \\
\hline CS 29527-015 & $00^{\mathrm{h}} 29^{\mathrm{m}} 10^{\varsigma} .5$ & $-19^{\circ} 10^{\prime} 07^{\prime \prime}$ & 14.25 & 13.29 & 13.08 & 13.05 & 0.02213 \\
\hline CS 30301-024 & $15^{\mathrm{h}} 08^{\mathrm{m}} 29.7$ & $-00^{\circ} 36^{\prime} 02^{\prime \prime}$ & 12.95 & 11.93 & 11.67 & 11.67 & 0.06527 \\
\hline CS 30302-145 & $19^{\mathrm{h}} 40^{\mathrm{m}} 52^{\mathrm{s}} .2$ & $-48^{\circ} 39^{\prime} 19^{\prime \prime}$ & 14.46 & 13.48 & 13.23 & 13.26 & 0.05343 \\
\hline CS 30339-069 & $00^{\mathrm{h}} 30^{\mathrm{m}} 15^{\mathrm{s}} .9$ & $-35^{\circ} 56^{\prime} 51^{\prime \prime}$ & 14.75 & 13.77 & 13.52 & 13.45 & 0.00904 \\
\hline CS 30344-070 & $22^{\mathrm{h}} 47^{\mathrm{m}} 23^{\mathrm{s}} .2$ & $-35^{\circ} 32^{\prime} 44^{\prime \prime}$ & 14.43 & 13.53 & 13.26 & 13.27 & 0.01305 \\
\hline CS 31061-032 & $02^{\mathrm{h}} 38^{\mathrm{m}} 43^{\mathrm{s}} .1$ & $+03^{\circ} 19^{\prime} 03^{\prime \prime}$ & 13.90 & 12.87 & 12.62 & 12.61 & 0.03727 \\
\hline HE 0148-2611 & $01^{\mathrm{h}} 50^{\mathrm{m}} 59^{\mathrm{s}} .5$ & $-25^{\circ} 57^{\prime} 02^{\prime \prime}$ & 14.45 & 13.55 & 13.30 & 13.30 & 0.01362 \\
\hline HE 1413-1954 & $14^{\mathrm{h}} 16^{\mathrm{m}} 04^{\mathrm{s}} .7$ & $-20^{\circ} 08^{\prime} 54^{\prime \prime}$ & 15.23 & 14.19 & 13.97 & 13.89 & 0.08681 \\
\hline LP $815-43$ & $20^{\mathrm{h}} 38^{\mathrm{m}} 13^{\mathrm{s}} .3$ & $-20^{\circ} 26^{\prime} 11^{\prime \prime}$ & 10.91 & 9.96 & 9.71 & 9.65 & 0.04514 \\
\hline
\end{tabular}

Table 3. An extract from the line-by-line Fe I and Fe II abundance table.

\begin{tabular}{lcccccccc}
\hline \hline Star & Ion & $\begin{array}{c}\lambda \\
(\mathrm{nm})\end{array}$ & $\log g f$ & $\begin{array}{c}E W \\
\mathrm{pm}\end{array}$ & $\begin{array}{c}\epsilon \\
\mathrm{BA}\end{array}$ & $\begin{array}{c}\epsilon \\
\text { ALI }\end{array}$ & $\begin{array}{c}\epsilon \\
\text { IRFM }\end{array}$ & $\begin{array}{c}\epsilon \\
\text { 3D }\end{array}$ \\
\hline BS 16023-046 & $\mathrm{Fe}$ I & 340.1519 & -2.059 & 14.60 & 4.85 & 5.01 & 5.04 & 4.89 \\
& $\mathrm{Fe} I$ & 340.7460 & -0.020 & 28.60 & 4.38 & 4.51 & 4.54 & 4.41 \\
& $\mathrm{Fe} I$ & 342.7119 & -0.098 & 29.40 & 4.50 & 4.47 & 4.61 & 4.63 \\
& $\mathrm{Fe} \mathrm{I}$ & 344.0989 & -0.958 & 76.50 & 4.77 & 4.92 & 4.95 & 4.76 \\
$\ldots$ & & & & & & & & \\
\hline
\end{tabular}

Notes. The full table is available at the CDS.

\section{Lithium abundance determination}

We determined $\mathrm{Li}$ equivalent widths in a similar fashion to Bonifacio et al. (2007). Synthetic line profiles were fitted to the observed profile, and the equivalent width $(E W)$ determined from the fitted synthetic profile. The $E W$ errors listed in Table 6 were obtained by means of Monte Carlo simulations, in which Poisson noise was added to a synthetic spectrum to ensure that it had the same $S / N$ as the observed spectrum. The Li abundance was determined by iteratively computing synthetic spectra of the Li doublet until the synthetic $E W$ matched the observed $E W$ to better than $1 \%$. The adopted atomic data were unchanged with respect to Bonifacio et al. (2007), and took account of hyperfine structure and isotopic components (a solar Li isotopic ratio was assumed). We henceforth refer to these abundances as "1D Li abundances" since Li abundances were derived using
1D atmosphere model and spectrosynthesis codes. One should avoid confusion with the 3D temperature scale, which indicates only that $3 \mathrm{D}$ effects have been taken into account in determining the $\mathrm{H} \alpha$-wing fitting temperature.

In addition, we determined, for the $3 D$ temperature scale only, what we refer to as "3D NLTE Li abundances". As described in Sect. 6.1, a grid of time-dependent 3D NLTE spectrosyntheses have been produced for the Li I $670.8 \mathrm{~nm}$ doublet, and used to independently determine $\mathrm{Li}$ abundances from the measured $E W$.

The uncertainties in the $\mathrm{Li}$ abundance measurements were largely dominated by the uncertainty in the temperature estimation. For further details, the reader is referred to Bonifacio et al. (2007). For the purpose of our analysis, a constant uncertainty of $\sigma_{A(\mathrm{Li})}=0.09$ was assumed. 
Table 4. Atmosphere parameters for the program stars using the different temperature estimators.

\begin{tabular}{|c|c|c|c|c|c|c|c|c|c|c|c|c|c|c|c|c|}
\hline Star & $\begin{array}{r}T_{\text {eff }} \\
\mathrm{K}\end{array}$ & $\begin{array}{l}\log g \\
\text { CGS }\end{array}$ & $\underset{\mathrm{BA}}{\mathrm{km} \mathrm{s}^{-1}}$ & {$[\mathrm{Fe} / \mathrm{H}]$} & $\begin{array}{r}T_{\text {eff }} \\
\mathrm{K}\end{array}$ & $\begin{array}{l}\log g \\
\text { CGS }\end{array}$ & $\begin{array}{c}\mathrm{km} \mathrm{s}^{-1} \\
\mathrm{ALI}\end{array}$ & {$[\mathrm{Fe} / \mathrm{H}]$} & $\begin{array}{r}T_{\mathrm{eff}} \\
\mathrm{K}\end{array}$ & $\begin{array}{r}\log g \\
\text { CGS }\end{array}$ & \begin{tabular}{l}
\multicolumn{1}{c}{$\xi$} \\
$\mathrm{km} \mathrm{s}^{-1}$ \\
$\mathrm{FM}$
\end{tabular} & {$[\mathrm{Fe} / \mathrm{H}]$} & $\begin{array}{r}T_{\text {eff }} \\
\mathrm{K}\end{array}$ & $\begin{array}{l}\log g \\
\text { CGS }\end{array}$ & $\underbrace{}_{3 \mathrm{Dm} \mathrm{s}} \mathrm{km}^{-1}$ & {$[\mathrm{Fe} / \mathrm{H}]$} \\
\hline BS 16023-046 & 6324 & 4.30 & 1.4 & -2.97 & 6527 & 4.60 & 1.4 & -2.84 & 6560 & 4.60 & 1.4 & -2.82 & 6401 & 4.50 & 1.4 & -2.94 \\
\hline BS $17570-063$ & 6078 & 4.50 & 0.6 & -3.05 & 6404 & 4.80 & 0.7 & -2.79 & 6315 & 4.70 & 0.7 & -2.86 & 6237 & 4.70 & 0.6 & -2.92 \\
\hline BS $17572-100$ & 6371 & 4.00 & 1.6 & -2.75 & 6504 & 4.40 & 1.5 & -2.62 & 6689 & 4.70 & 1.5 & -2.52 & 6425 & 4.30 & 1.5 & -2.72 \\
\hline CS 22177-009 & 6177 & 4.30 & 1.3 & -3.17 & 6415 & 4.70 & 1.3 & -2.99 & 6479 & 4.70 & 1.3 & -2.96 & 6284 & 4.50 & 1.2 & -3.08 \\
\hline CS 22188-033 & 6129 & 4.40 & 1.4 & -3.03 & 6411 & 4.90 & 1.3 & -2.85 & 6281 & 4.50 & 1.4 & -2.98 & 6242 & 4.70 & 1.2 & -2.97 \\
\hline CS 22882-027 & - & - & - & - & - & - & - & - & - & - & - & - & 6714 & 4.70 & 1.4 & -2.40 \\
\hline CS 22888-031 & 5925 & 4.50 & 0.7 & -3.47 & 6304 & 5.10 & 0.7 & -3.18 & 6480 & 5.20 & 1.0 & -3.07 & 6090 & 4.90 & 0.4 & -3.33 \\
\hline CS 22948-093 & 6365 & 4.25 & 1.3 & -3.31 & 6551 & 4.50 & 1.3 & -3.15 & 6577 & 4.70 & 1.2 & -3.18 & 6450 & 4.40 & 1.3 & -3.24 \\
\hline CS $22950-173$ & 6335 & 4.20 & 1.4 & -2.78 & 6506 & 4.50 & 1.4 & -2.61 & 6353 & 4.20 & 1.4 & -2.73 & 6415 & 4.40 & 1.4 & -2.69 \\
\hline CS 22953-037 & 6325 & 4.25 & 1.4 & -2.91 & 6515 & 4.50 & 1.4 & -2.75 & 6557 & 4.45 & 1.4 & -2.76 & 6416 & 4.40 & 1.4 & -2.84 \\
\hline CS 22965-054 & 6245 & 4.00 & 1.5 & -2.90 & 6398 & 4.20 & 1.5 & -2.78 & 6417 & 4.20 & 1.5 & -2.79 & 6312 & 4.10 & 1.4 & -2.86 \\
\hline CS 22966-011 & 6049 & 4.40 & 1.1 & -3.22 & 6345 & 4.90 & 1.1 & -2.96 & 6302 & 4.80 & 1.1 & -3.01 & 6166 & 4.70 & 1.0 & -3.09 \\
\hline CS 29491-084 & 6285 & 4.00 & 1.7 & -3.04 & 6453 & 4.20 & 1.8 & -2.90 & 6425 & 4.20 & 1.8 & -2.94 & 6381 & 4.10 & 1.8 & -2.97 \\
\hline CS 29499-060 & 6349 & 4.10 & 1.5 & -2.66 & 6493 & 4.40 & 1.4 & -2.56 & 6560 & 4.50 & 1.5 & -2.56 & 6428 & 4.30 & 1.5 & -2.62 \\
\hline CS 29506-007 & 6285 & 4.20 & 1.6 & -2.88 & 6478 & 4.40 & 1.7 & -2.70 & 6515 & 4.40 & 1.7 & -2.71 & 6397 & 4.30 & 1.7 & -2.81 \\
\hline CS 29506-090 & 6287 & 4.20 & 1.4 & -2.83 & 6480 & 4.55 & 1.4 & -2.67 & 6557 & 4.45 & 1.5 & -2.63 & 6367 & 4.30 & 1.4 & -2.77 \\
\hline CS 29514-007 & 6281 & 4.10 & 1.5 & -2.80 & 6448 & 4.40 & 1.5 & -2.66 & 6351 & 4.30 & 1.4 & -2.79 & 6361 & 4.30 & 1.5 & -2.76 \\
\hline CS 29516-028 & 5839 & 4.40 & 1.2 & -3.52 & 6198 & 5.00 & 1.2 & -3.19 & 5994 & 4.70 & 1.2 & -3.39 & 6004 & 4.90 & 0.9 & -3.33 \\
\hline CS 29518-020 & 6127 & 4.30 & 1.8 & -2.86 & 6368 & 4.80 & 1.8 & -2.67 & 6471 & 4.90 & 1.9 & -2.60 & 6213 & 4.60 & 1.8 & -2.79 \\
\hline CS 29518-043 & 6376 & 4.25 & 1.3 & -3.25 & 6566 & 4.40 & 1.4 & -3.10 & 6537 & 4.25 & 1.4 & -3.16 & 6489 & 4.30 & 1.4 & -3.17 \\
\hline CS 29527-015 & 6276 & 4.00 & 1.6 & -3.53 & 6426 & 4.40 & 1.6 & -3.37 & 6578 & 4.50 & 1.7 & -3.31 & 6325 & 4.30 & 1.6 & -3.49 \\
\hline CS 30301-024 & 6375 & 4.00 & 1.6 & -2.71 & 6494 & 4.50 & 1.6 & -2.60 & 6581 & 4.50 & 1.6 & -2.60 & 6400 & 4.30 & 1.5 & -2.69 \\
\hline CS 30302-145 & 6403 & 4.30 & 1.8 & -3.02 & 6597 & 4.50 & 1.8 & -2.88 & 6645 & 4.50 & 1.9 & -2.88 & 6497 & 4.40 & 1.8 & -2.94 \\
\hline CS 30339-069 & 6253 & 4.00 & 1.4 & -3.09 & 6402 & 4.40 & 1.4 & -2.93 & 6375 & 4.40 & 1.3 & -2.98 & 6304 & 4.30 & 1.3 & -3.04 \\
\hline CS 30344-070 & 6302 & 4.10 & 1.6 & -3.02 & 6477 & 4.30 & 1.7 & -2.85 & 6568 & 4.40 & 1.8 & -2.82 & 6407 & 4.20 & 1.7 & -2.92 \\
\hline CS 31061-032 & 6369 & 4.25 & 1.4 & -2.62 & 6555 & 4.50 & 1.5 & -2.48 & 6405 & 4.25 & 1.4 & -2.58 & 6466 & 4.40 & 1.5 & -2.56 \\
\hline HE 0148-2611 & 6400 & 4.10 & 1.5 & -3.18 & 6565 & 4.30 & 1.6 & -3.06 & 6606 & 4.20 & 1.6 & -3.07 & 6505 & 4.20 & 1.6 & -3.12 \\
\hline HE $1413-1954$ & 6302 & 3.80 & 1.7 & -3.50 & 6448 & 4.10 & 1.7 & -3.39 & 6716 & 4.40 & 1.8 & -3.22 & 6370 & 4.00 & 1.7 & -3.47 \\
\hline LP 815-43 & 6453 & 3.80 & 1.7 & -2.88 & 6579 & 4.10 & 1.7 & -2.81 & 6630 & 4.10 & 1.7 & -2.77 & 6578 & 4.00 & 1.7 & -2.80 \\
\hline
\end{tabular}

Notes. Parameters for CS 22882-027 were derived only for the 3D temperature scale.

\subsection{D and NLTE corrections}

We originally planned to determine the effects of both atmosphere hydrodynamics and any departure from LTE in a consistent manner, and thus computed a set of time-dependent 3D NLTE syntheses of the Li doublet over a grid of suitable 3D models, to construct a set of curves of growth (COG) for the doublet $E W$. Details of the computation of the 3D NLTE lithium doublet synthesis are covered in Appendix A.

The model parameters covered by the COG grid are listed in Table 7. Once the COG grid was computed, we decided to also derive 3D NLTE lithium abundances directly, by identifying the $E W$-to-abundance relation that most closely fitted the computed values, and applying it to our observed $E W$. This was accomplished by either interpolating in the $T_{\text {eff }}, \log g,[\mathrm{Fe} / \mathrm{H}]$, and $E W$ grid (and possibly extrapolating out of it), or by determining a best fitting analytical function in the form $A(\mathrm{Li})=f\left(T_{\text {eff }}\right.$, $\log g,[\mathrm{Fe} / \mathrm{H}], E W)$ and applying it to the observed parameters and lithium doublet $E W$. We pursued both of these approaches.

We found the functional-fit method to be the superior of the two, both because of its higher accuracy and for greater ease of use. Its functional-form approach condenses the 3D NLTE abundance determination into a formula that can be hard-coded into any program, eliminating the need to carry over the true grid of computed points. Details on the fit calculation, as well as the chosen functional form and coefficients, are available in Appendix $\mathrm{B}^{5}$.

As mentioned above, we also performed an interpolation over the COG grid. The main problem in producing a suitable

\footnotetext{
5 The corresponding IDL functions are also available on-line at http://mygepi.obspm. fr/ sbordone/fitting.html or by email request to the authors.
}

interpolation lies is that the grid is non-rectangular, which has two different causes. First, in the $\mathrm{CO}^{5} \mathrm{BOLD}$ hydrodynamical models $T_{\text {eff }}$ is not set a priori, rather, the entropy of the material entering through the bottom of the computational box is the fixed quantity. The true $T_{\text {eff }}$ is determined after snapshot selection, and usually varies across an interval of $\pm 100 \mathrm{~K}$ centered on the desired value for the models we employed. As a consequence, it is impossible to build a grid of CO ${ }^{5}$ BOLD models with exactly the same temperature but, for example, different metallicity. Secondly, varying the stellar parameters naturally alters the relationship between $A(\mathrm{Li})$ and $E W$, so that the range of $E W$ in the $\mathrm{COG}$ corresponding to interesting values of $A(\mathrm{Li})$ will vary from model to model.

To simplify the task, we took advantage of the limited sensitivity of the $670.8 \mathrm{~nm}$ Li doublet to both gravity and metallicity. Thus, we decided to assume $[\mathrm{Fe} / \mathrm{H}]=-3$ throughout the interpolation, and to avoid interpolating in gravity by always choosing the closest value to the derived gravity between $\log g=4$ and $\log g=4.5$. This choice was also justified by the limited extension in both parameters of our sample. This reduced the problem to interpolating in an irregularly spaced two-dimensional grid in $T_{\text {eff }}$ and $E W$. Delaunay triangulation ${ }^{6}$ and quintic polynomial interpolation were then used to derive $A(\mathrm{Li})$.

Figures 6 and 7 show the difference between $A(\mathrm{Li})$ as determined by means of the analytical fit $\left(A(\mathrm{Li})_{\mathrm{f}}\right)$ and by interpolation

\footnotetext{
6 Delaunay triangulation is a method of triangulation of a set $\mathrm{P}$ of points in a plane defined as the triangulation for which no element of $P$ lies within the circumcircle of each triangle, except for the triangle vertexes. It is often used to model surfaces that are sampled on irregular grids (e.g., elevations in geography). The built-in IDL functions triangulate and trigrid have been used to produce the triangulation and the interpolation based on it.
} 
L. Sbordone et al.: The metal-poor end of the Spite plateau. I.

Table 5. Fe I and Fe II mean abundances, as well as their associated $\sigma$ for the four temperature scales.

\begin{tabular}{|c|c|c|c|c|c|c|c|c|c|c|c|c|c|c|c|c|}
\hline Star & $\mathrm{Fe} \mathrm{I}$ & \multicolumn{2}{|c|}{ BA } & $\sigma$ & \multicolumn{3}{|c|}{ ALI } & $\sigma$ & $\mathrm{Fe} I$ & $\sigma$ & $\begin{array}{l}\text { Fe II } \\
M\end{array}$ & $\sigma$ & \multicolumn{3}{|c|}{$3 \mathrm{D}$} & $\sigma$ \\
\hline BS 16023-046 & 4.53 & 0.108 & 4.50 & 0.135 & 4.66 & 0.113 & 4.64 & 0.135 & 4.68 & 0.115 & 4.64 & 0.135 & 4.56 & 0.108 & 4.58 & 0.136 \\
\hline BS $17570-063$ & 4.45 & 0.136 & 4.51 & 0.062 & 4.71 & 0.156 & 4.66 & 0.062 & 4.64 & 0.151 & 4.62 & 0.061 & 4.58 & 0.144 & 4.61 & 0.062 \\
\hline CS 22177-009 & 4.33 & 0.101 & 4.37 & 0.123 & 4.51 & 0.121 & 4.54 & 0.126 & 4.54 & 0.114 & 4.55 & 0.127 & 4.42 & 0.116 & 4.46 & 0.123 \\
\hline CS 22188-033 & 4.47 & 0.104 & 4.44 & 0.030 & 4.65 & 0.123 & 4.66 & 0.032 & 4.52 & 0.097 & 4.49 & 0.032 & 4.53 & 0.110 & 4.57 & 0.027 \\
\hline CS 22888-031 & 4.03 & 0.148 & 4.04 & 0.125 & 4.32 & 0.164 & 4.30 & 0.132 & 4.43 & 0.173 & 4.35 & 0.137 & 4.17 & 0.154 & 4.21 & 0.126 \\
\hline CS 22948-093 & 4.19 & 0.133 & 4.20 & 0.144 & 4.35 & 0.132 & 4.31 & 0.146 & 4.32 & 0.129 & 4.38 & 0.145 & 4.26 & 0.134 & 4.26 & 0.146 \\
\hline CS 22953-037 & 4.59 & 0.126 & 4.62 & 0.124 & 4.75 & 0.145 & 4.74 & 0.125 & 4.74 & 0.136 & 4.72 & 0.125 & 4.66 & 0.128 & 4.69 & 0.125 \\
\hline CS 22965-054 & 4.60 & 0.136 & 4.62 & 0.089 & 4.72 & 0.141 & 4.71 & 0.091 & 4.71 & 0.140 & 4.71 & 0.091 & 4.64 & 0.138 & 4.67 & 0.083 \\
\hline CS 22966-011 & 4.28 & 0.106 & 4.30 & 0.120 & 4.54 & 0.131 & 4.53 & 0.121 & 4.49 & 0.119 & 4.48 & 0.121 & 4.41 & 0.118 & 4.44 & 0.119 \\
\hline CS 29491-084 & 4.46 & 0.130 & 4.49 & 0.119 & 4.60 & 0.134 & 4.58 & 0.119 & 4.56 & 0.133 & 4.57 & 0.119 & 4.53 & 0.132 & 4.53 & 0.119 \\
\hline CS 29499-060 & 4.84 & 0.114 & 4.82 & 0.099 & 4.94 & 0.127 & 4.96 & 0.097 & 4.94 & 0.113 & 4.98 & 0.100 & 4.88 & 0.120 & 4.89 & 0.100 \\
\hline CS 29506-007 & 4.62 & 0.116 & 4.67 & 0.183 & 4.80 & 0.125 & 4.77 & 0.186 & 4.79 & 0.124 & 4.77 & 0.186 & 4.69 & 0.118 & 4.72 & 0.186 \\
\hline CS 29506-090 & 4.67 & 0.110 & 4.71 & 0.109 & 4.83 & 0.109 & 4.82 & 0.113 & 4.87 & 0.122 & 4.82 & 0.113 & 4.73 & 0.114 & 4.76 & 0.109 \\
\hline CS 29514-007 & 4.70 & 0.130 & 4.66 & 0.084 & 4.84 & 0.136 & 4.80 & 0.087 & 4.71 & 0.132 & 4.75 & 0.078 & 4.74 & 0.133 & 4.74 & 0.086 \\
\hline CS 29518-020 & 4.64 & 0.092 & 4.62 & 0.138 & 4.83 & 0.115 & 4.83 & 0.139 & 4.90 & 0.125 & 4.87 & 0.141 & 4.71 & 0.100 & 4.74 & 0.139 \\
\hline CS 29518-043 & 4.25 & 0.126 & 4.28 & 0.129 & 4.40 & 0.128 & 4.36 & 0.133 & 4.34 & 0.124 & 4.30 & 0.133 & 4.33 & 0.125 & 4.31 & 0.133 \\
\hline CS 29527-015 & 3.97 & 0.143 & 3.98 & 0.179 & 4.13 & 0.143 & 4.15 & 0.179 & 4.19 & 0.142 & 4.19 & 0.179 & 4.01 & 0.145 & 4.09 & 0.179 \\
\hline CS 30301-024 & 4.79 & 0.118 & 4.73 & 0.106 & 4.90 & 0.119 & 4.92 & 0.108 & 4.90 & 0.119 & 4.92 & 0.108 & 4.81 & 0.125 & 4.85 & 0.102 \\
\hline CS 30302-145 & 4.48 & 0.160 & 4.48 & 0.102 & 4.62 & 0.160 & 4.58 & 0.107 & 4.62 & 0.160 & 4.58 & 0.107 & 4.56 & 0.163 & 4.53 & 0.103 \\
\hline CS 30339-069 & 4.41 & 0.188 & 4.39 & 0.098 & 4.57 & 0.179 & 4.56 & 0.101 & 4.52 & 0.177 & 4.55 & 0.096 & 4.46 & 0.189 & 4.51 & 0.096 \\
\hline CS 30344-070 & 4.48 & 0.121 & 4.51 & 0.104 & 4.65 & 0.123 & 4.61 & 0.101 & 4.68 & 0.125 & 4.65 & 0.101 & 4.58 & 0.122 & 4.56 & 0.102 \\
\hline CS 31061-032 & 4.88 & 0.137 & 4.88 & 0.062 & 5.02 & 0.132 & 4.98 & 0.067 & 4.92 & 0.123 & 4.89 & 0.062 & 4.94 & 0.125 & 4.93 & 0.068 \\
\hline HE 0148-2611 & 4.32 & 0.112 & 4.31 & 0.048 & 4.44 & 0.118 & 4.41 & 0.051 & 4.43 & 0.116 & 4.41 & 0.051 & 4.38 & 0.117 & 4.36 & 0.050 \\
\hline HE $1413-1954$ & 4.00 & 0.118 & 4.01 & 0.167 & 4.11 & 0.118 & 4.13 & 0.167 & 4.28 & 0.118 & 4.27 & 0.168 & 4.03 & 0.120 & 4.09 & 0.168 \\
\hline LP 815-43 & 4.62 & 0.088 & 4.58 & 0.051 & 4.69 & 0.089 & 4.71 & 0.052 & 4.73 & 0.091 & 4.71 & 0.052 & 4.70 & 0.090 & 4.67 & 0.052 \\
\hline
\end{tabular}

Table 6. Li I $670.8 \mathrm{~nm} E W$ and errors, and lithium abundances using the different parameter sets.

\begin{tabular}{|c|c|c|c|c|c|c|c|c|c|c|c|}
\hline \multirow[t]{2}{*}{ Star } & \multirow[t]{2}{*}{$\begin{array}{c}E W \\
\mathrm{pm}\end{array}$} & \multirow[t]{2}{*}{$\begin{array}{c}\text { Error } \\
\mathrm{pm}\end{array}$} & $\begin{array}{c}A(\mathrm{Li}) \\
(a)\end{array}$ & $\begin{array}{c}A(\mathrm{Li}) \\
(b)\end{array}$ & $\begin{array}{c}A(\mathrm{Li}) \\
(\text { a })\end{array}$ & $\begin{array}{c}A(\mathrm{Li}) \\
(b)\end{array}$ & $\begin{array}{c}A(\mathrm{Li}) \\
(a)\end{array}$ & $\begin{array}{c}A(\mathrm{Li}) \\
(b)\end{array}$ & $\begin{array}{c}A(\mathrm{Li}) \\
(a)\end{array}$ & $\begin{array}{c}A(\mathrm{Li}) \\
(b)\end{array}$ & $\begin{array}{c}A(\mathrm{Li}) \\
(c)\end{array}$ \\
\hline & & & \multicolumn{2}{|c|}{ (a) $\mathrm{BA}$} & \multicolumn{2}{|c|}{ ALI } & \multicolumn{2}{|c|}{ IRFM } & \multicolumn{3}{|c|}{$3 \mathrm{D}$} \\
\hline BS 16023-046 & 1.93 & 0.06 & 2.145 & 2.138 & 2.271 & 2.257 & 2.292 & 2.278 & 2.193 & 2.181 & 2.179 \\
\hline BS $17570-063$ & 1.76 & 0.04 & 1.930 & 1.928 & 2.148 & 2.132 & 2.091 & 2.077 & 2.038 & 2.025 & 2.029 \\
\hline BS $17572-100$ & 1.81 & 0.04 & 2.152 & 2.149 & 2.232 & 2.219 & 2.340 & 2.329 & 2.184 & 2.174 & 2.166 \\
\hline CS 22177-009 & 2.42 & 0.03 & 2.153 & 2.153 & 2.309 & 2.293 & 2.339 & 2.323 & 2.224 & 2.214 & 2.209 \\
\hline CS 22188-033 & 0.78 & 0.05 & 1.577 & 1.577 & 1.750 & 1.735 & 1.665 & 1.657 & 1.648 & 1.636 & 1.665 \\
\hline CS 22888-031 & 1.87 & 0.04 & 1.846 & 1.851 & 2.104 & 2.081 & 2.214 & 2.199 & 1.961 & 1.940 & 1.976 \\
\hline CS 22948-093 & 1.19 & 0.06 & 1.935 & 1.930 & 2.047 & 2.034 & 2.051 & 2.039 & 1.988 & 1.977 & 1.989 \\
\hline CS $22950-173$ & 2.11 & 0.09 & 2.199 & 2.193 & 2.307 & 2.293 & 2.212 & 2.205 & 2.250 & 2.238 & 2.230 \\
\hline $\mathrm{CS} 2$ & 1.95 & 0.03 & 2.151 & 2.145 & 2.272 & 2.258 & 2.278 & 2.265 & 2.211 & 2.200 & 2.194 \\
\hline CS 2296 & 2.21 & 0.06 & 2.161 & 2.164 & 2.263 & 2.255 & 2.277 & 2.268 & 2.206 & 2.203 & 2.185 \\
\hline CS 2296 & 1.37 & 0.05 & 1.788 & 1.792 & 1.983 & 1.966 & 1.945 & 1.929 & 1.867 & 1.855 & 1.869 \\
\hline CS 29491-084 & 1.77 & 0.07 & 2.080 & 2.083 & 2.189 & 2.180 & 2.171 & 2.163 & 2.142 & 2.138 & 2.128 \\
\hline CS 29499-060 & 2.07 & 0.06 & 2.201 & 2.196 & 2.322 & 2.309 & 2.311 & 2.297 & 2.250 & 2.239 & 2.231 \\
\hline CS 29506-007 & 2.05 & 0.04 & 2.149 & 2.146 & 2.275 & 2.262 & 2.298 & 2.285 & 2.223 & 2.214 & 2.205 \\
\hline CS 29506-090 & 1.85 & 0.05 & 2.102 & 2.098 & 2.225 & 2.211 & 2.274 & 2.261 & 2.156 & 2.147 & 2.137 \\
\hline CS 29514-007 & 2.33 & 0.09 & 2.211 & 2.209 & 2.320 & 2.307 & 2.231 & 2.222 & 2.263 & 2.254 & 2.241 \\
\hline CS 29516-028 & 2.41 & 0.12 & 1.904 & 1.919 & 2.159 & 2.136 & 2.000 & 1.990 & 2.025 & 2.004 & 2.035 \\
\hline CS 29518-020 & 2.10 & 0.11 & 2.052 & 2.053 & 2.211 & 2.195 & 2.276 & 2.260 & 2.109 & 2.099 & 2.091 \\
\hline CS 29518-043 & 1.72 & 0.11 & 2.121 & 2.115 & 2.238 & 2.225 & 2.204 & 2.193 & 2.193 & 2.182 & 2.183 \\
\hline CS 29527-015 & 1.86 & 0.06 & 2.091 & 2.098 & 2.188 & 2.177 & 2.272 & 2.259 & 2.121 & 2.116 & 2.113 \\
\hline CS 30301-024 & 1.77 & 0.06 & 2.143 & 2.140 & 2.213 & 2.200 & 2.250 & 2.237 & 2.157 & 2.148 & 2.139 \\
\hline CS 30302-145 & 1.54 & 0.11 & 2.086 & 2.077 & 2.203 & 2.190 & 2.215 & 2.203 & 2.143 & 2.131 & 2.138 \\
\hline CS 30339-069 & 2.04 & 0.11 & 2.125 & 2.130 & 2.223 & 2.212 & 2.204 & 2.194 & 2.157 & 2.151 & 2.140 \\
\hline CS 30344-070 & 1.68 & 0.09 & 2.064 & 2.063 & 2.177 & 2.166 & 2.231 & 2.218 & 2.135 & 2.128 & 2.120 \\
\hline CS 31061-032 & 2.10 & 0.06 & 2.221 & 2.213 & 2.336 & 2.322 & 2.250 & 2.241 & 2.282 & 2.269 & 2.262 \\
\hline HE 0148-2611 & 1.29 & 0.09 & 2.000 & 1.996 & 2.100 & 2.089 & 2.113 & 2.102 & 2.065 & 2.056 & 2.063 \\
\hline HE $1413-1954$ & 1.59 & 0.14 & 2.035 & 2.047 & 2.129 & 2.123 & 2.285 & 2.274 & 2.079 & 2.080 & 2.070 \\
\hline LP 815-43 & 1.89 & 0.06 & 2.229 & 2.228 & 2.302 & 2.292 & 2.334 & 2.324 & 2.304 & 2.296 & 2.296 \\
\hline
\end{tabular}

Notes. For the BA, ALI, and IRFM temperature scales, we list 1D LTE and 1D NLTE A(Li). For the 3D temperature scale, we list 1D LTE and NLTE, as well as 3D NLTE $A(\mathrm{Li})$. (a) 1D LTE value. (b) 1D value with NLTE correction applied. (c) 3D NLTE calculation. 


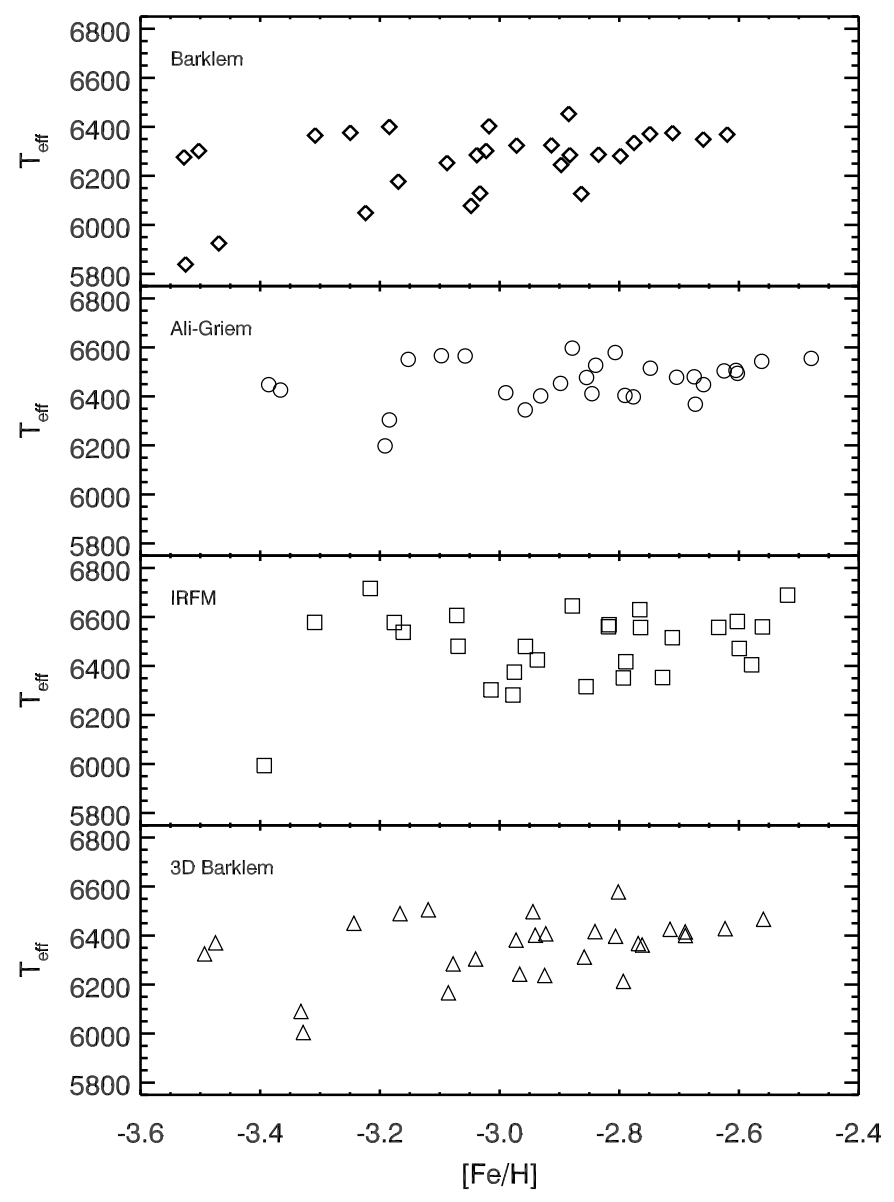

Fig. 5. Effective temperatures for different estimators, plotted against $[\mathrm{Fe} / \mathrm{H}]$ (as derived using the temperature in the panel). Top to bottom: BA temperatures, then ALI, IRFM and 3D.
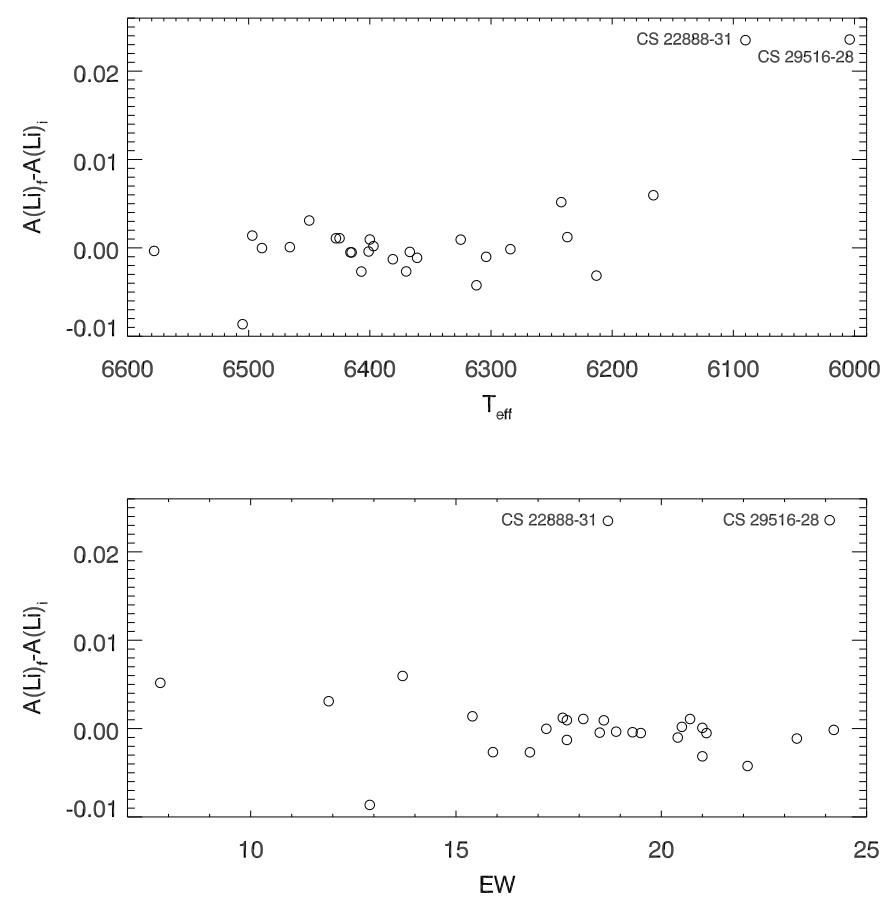

Fig. 6. Difference between $A(\mathrm{Li})_{\mathrm{f}}$ and $A(\mathrm{Li})_{\mathrm{i}}$ plotted against $T_{\text {eff }}$ and the Li doublet $E W$. The two "outliers" are labeled.

$\left(A(\mathrm{Li})_{\mathrm{i}}\right)$, respectively, plotted against relevant quantities, on the 3D temperature scale. Most stars show an excellent concordance
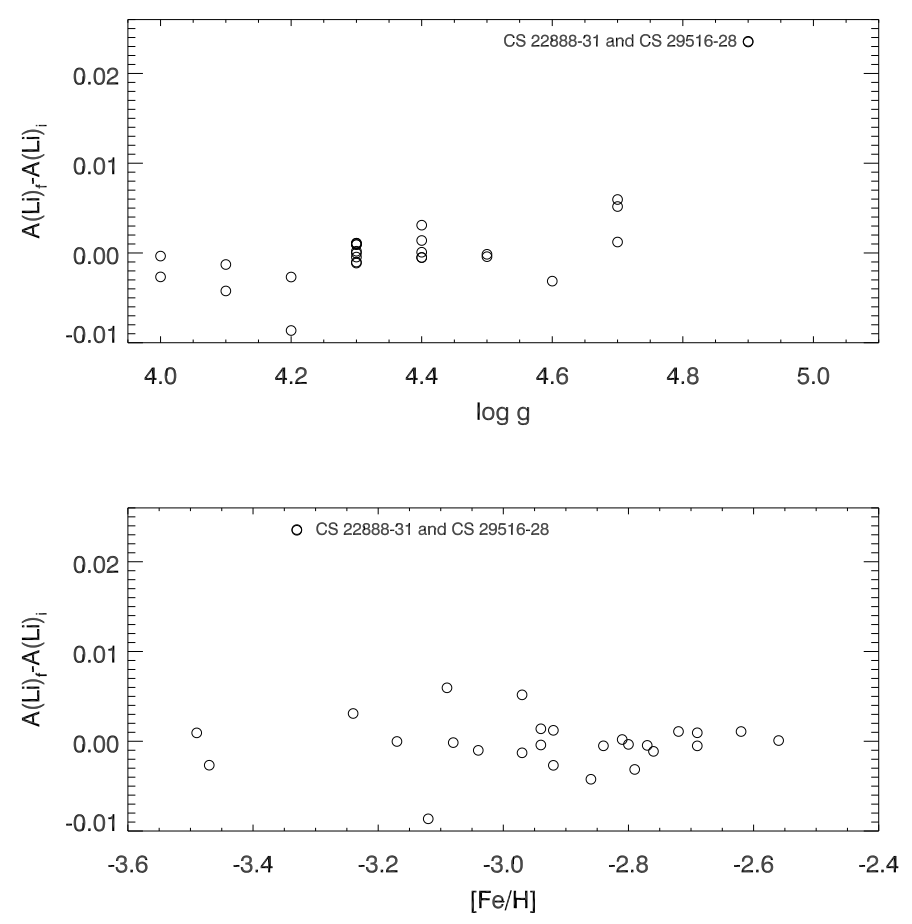

Fig. 7. Same as in Fig. 6 but now plotting against $\log g$ and $[\mathrm{Fe} / \mathrm{H}]$.

Table 7. Parameters of the models in the 3D $\mathrm{CO}^{5} \mathrm{BOLD}$ and $1 \mathrm{D}$ LHD grids used in the 3D NLTE Li abundances, and in the computation of NLTE corrections.

\begin{tabular}{lllcc}
\hline \hline $\begin{array}{l}T_{\text {eff }} \\
\mathrm{K}\end{array}$ & $\begin{array}{l}\log g \\
\operatorname{cgs}\end{array}$ & {$[\mathrm{Fe} / \mathrm{H}]$} & $A(\mathrm{Li})^{a}$ & $\begin{array}{c}E W^{b} \\
\mathrm{pm}\end{array}$ \\
\hline 5472. & 4.00 & -2.0 & $0.90-2.10$ & $5.71-69.71$ \\
5479. & 4.50 & -2.0 & $0.90-2.10$ & $5.94-72.80$ \\
5505. & 3.50 & -2.0 & $0.90-2.10$ & $4.98-61.94$ \\
5846. & 4.00 & -3.0 & $1.30-2.50$ & $6.67-76.04$ \\
5856. & 4.00 & -2.0 & $1.30-2.50$ & $6.79-77.83$ \\
5861. & 3.50 & -2.0 & $1.30-2.50$ & $6.28-72.67$ \\
5923. & 4.50 & -2.0 & $1.30-2.50$ & $6.17-73.16$ \\
5924. & 4.50 & -3.0 & $1.30-2.50$ & $5.76-68.94$ \\
6269. & 4.00 & -3.0 & $1.30-2.50$ & $3.29-43.33$ \\
6272. & 4.50 & -3.0 & $1.30-2.50$ & $3.32-43.83$ \\
6278. & 4.00 & -2.0 & $1.70-2.90$ & $8.05-86.35$ \\
6287. & 3.50 & -2.0 & $1.70-2.90$ & $7.42-81.33$ \\
6323. & 4.50 & -2.0 & $1.70-2.90$ & $7.71-84.35$ \\
6408. & 4.00 & -3.0 & $1.30-2.50$ & $2.68-36.35$ \\
6533. & 4.50 & -2.0 & $2.10-3.30$ & $13.55-119.59$ \\
6534. & 4.00 & -2.0 & $2.10-3.30$ & $12.87-118.58$ \\
6556. & 4.50 & -3.0 & $1.30-2.50$ & $2.16-30.20$ \\
\hline
\end{tabular}

Notes. (a) Minimum and maximum $A(\mathrm{Li})$ covered in the COG. (b) Minimum and maximum $E W$ for the Li doublet in the COG.

between the two methods, but two outliers exist, CS 29516028 and CS 22888-031. These two stars have the lowest temperatures among all the stars in the sample (these temperatures are still within the computed grid). However, they also have the highest gravity in the sample, which requires extrapolation, since the grid has a limiting gravity of $\log g$ of 4.5 . While the functional fit is indeed extrapolated, the simplified interpolation assumes $\log g=4.5$ in this instance; the discrepancy between the two methods does not however exceed 0.023 dex in $A(\mathrm{Li})$, which is negligible for our purpose. All the remaining stars exhibit discrepancies not exceeding 0.01 dex. 
L. Sbordone et al.: The metal-poor end of the Spite plateau. I.
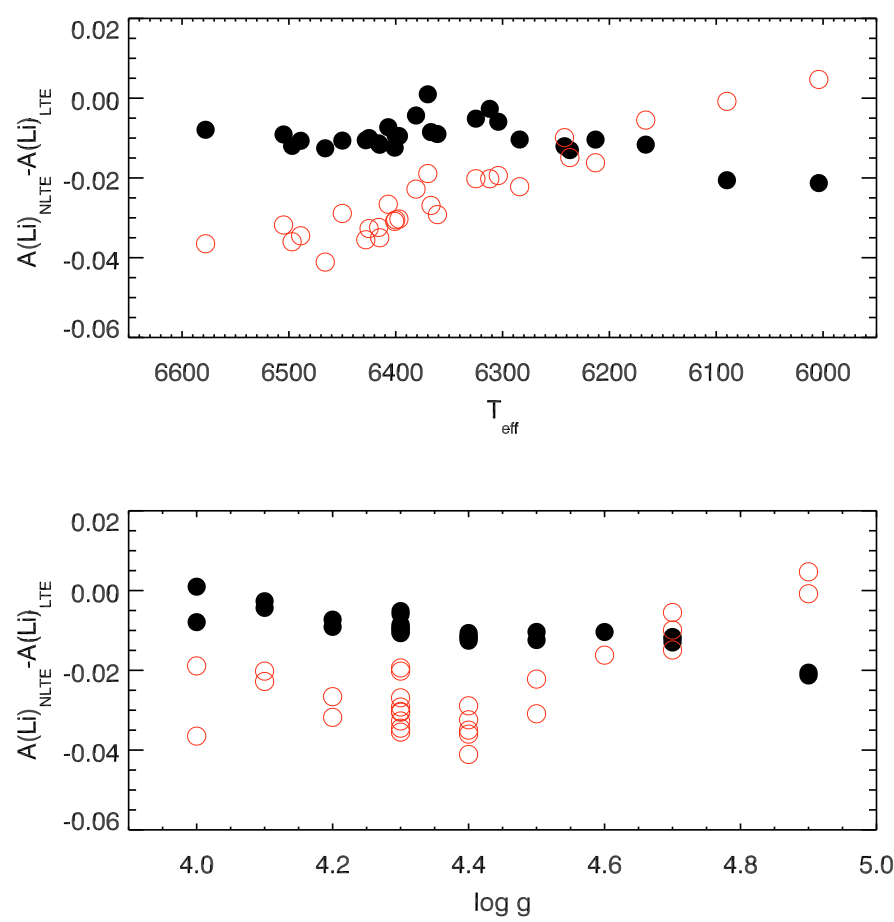

Fig. 8. The NLTE correction $\left(A(\mathrm{Li})_{1 \mathrm{D}, \mathrm{NLTE}}-A(\mathrm{Li})_{1 \mathrm{D}, \mathrm{LTE}}\right)$, computed for each star using our model atom (black filled dots) along with the Carlsson et al. (1994) values (red open circles), plotted against $T_{\text {eff }}$ and $\log g$. The 3D temperature scale is assumed.

A parallel grid of COG was produced using LHD models sharing the same parameters as the $C 0^{5} \mathrm{BOLD}$ ones. The $1 \mathrm{D}$ syntheses were produced both including and neglecting NLTE effects, for the specific purpose of deriving a grid of NLTE corrections applicable to our 1D Li abundances. For comparison, Fig. 8 shows our 1D NLTE corrections (for the 3D temperature scale) versus $T_{\text {eff }}$ and $\log g$, together with the corresponding values obtained by using the Carlsson et al. (1994) NLTE corrections, while Fig. 9 shows a similar comparison using the updated calculations by Lind et al. (2009a). Since Lind et al. (2009a) corrections are defined down to $[\mathrm{Fe} / \mathrm{H}]=-3$, in Fig. $9[\mathrm{Fe} / \mathrm{H}]=-3$ is assumed for all stars both in computing our NLTE correction and in computing those based on Lind et al. (2009a) scale. Trends with effective temperature and gravity are extremely similar for our corrections and those of Lind et al. (2009a), but a very uniform offset of about 0.03 dex is present between the two set of corrections. The origin of this offset is probably the different sets of underlying atmosphere models. Since the offset is quite uniform across the sample, using either set of corrections is of no consequence on the scientific output of the present work.

\section{Results}

We decided to adopt the 3D temperature scale (and its derived parameters) together with the 3D NLTE Li abundance set as our preferred values, and henceforth, when not otherwise specified, we will refer to these.

\subsection{Sensitivity to the adopted $T_{\text {eff }}$ scale}

One of the most remarkable results of this work is that, although the choice of temperature scale alters the parameters and derived $\mathrm{Li}$ abundance of the stars, it does not change the general picture
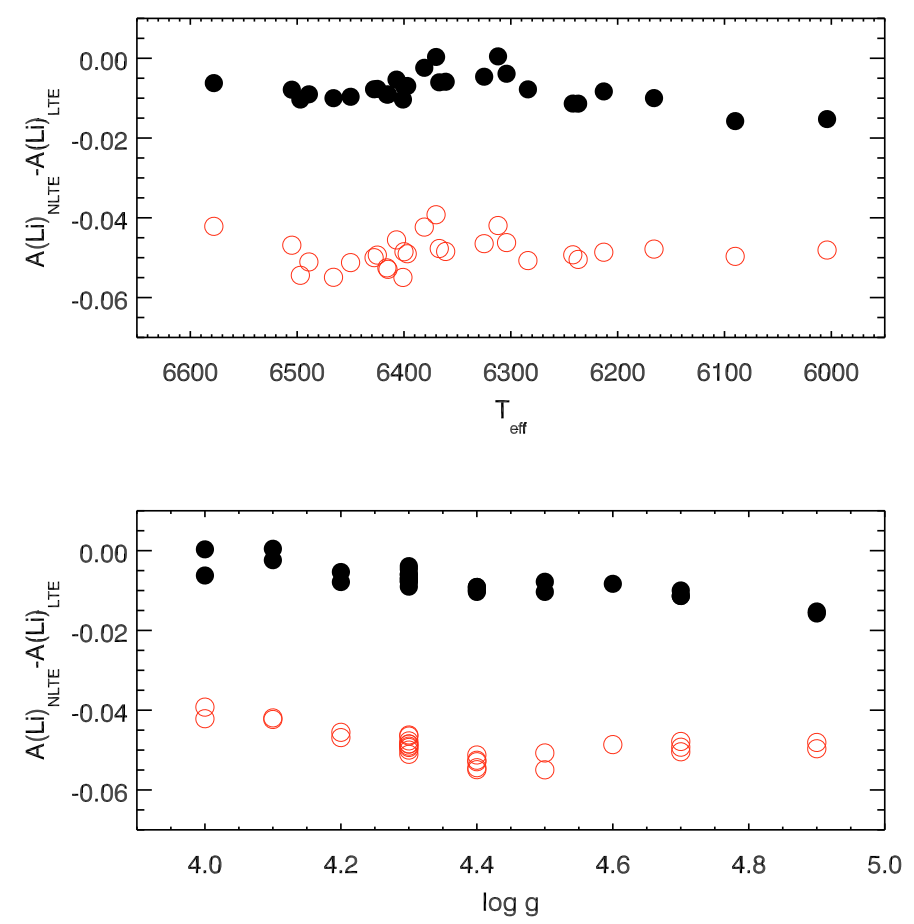

Fig. 9. The NLTE correction $\left(A(\mathrm{Li})_{1 \mathrm{D}, \mathrm{NLTE}}-A(\mathrm{Li})_{1 \mathrm{D}, \mathrm{LTE}}\right)$, computed for each star using our model atom (black filled dots) along with the Lind et al. (2009a) values (red open circles), plotted against $T_{\text {eff }}$ and $\log g$. The $3 \mathrm{D}$ temperature scale is assumed, and $[\mathrm{Fe} / \mathrm{H}]=-3$ is imposed for all stars.

Table 8. Kendall rank correlation probability, intercepts, and slopes of the linear fit and standard deviation of the slope for the plots shown in Figs. 12 and 10.

\begin{tabular}{lccc}
\hline \hline $\begin{array}{l}\text { Parameter } \\
\text { set }\end{array}$ & $\begin{array}{c}\text { Correlation } \\
\text { probability }\end{array}$ & $\begin{array}{c}\text { Linear fit } \\
\text { intercept }\end{array}$ & $\begin{array}{c}\text { Linear fit } \\
\text { slope }\end{array}$ \\
\hline$A(\mathrm{Li})$ vs. $T_{\text {eff }}$ & & & \\
BA & 0.978 & $-3.038 \pm 1.542$ & $8.17 \mathrm{e}-4 \pm 2.33 \mathrm{e}-4$ \\
ALI & 0.962 & $-7.917 \pm 10.83$ & $1.57 \mathrm{e}-3 \pm 1.00 \mathrm{e}-3$ \\
IRFM & 0.993 & $-2.133 \pm 1.448$ & $6.68 \mathrm{e}-4 \pm 2.13 \mathrm{e}-4$ \\
3D & 0.990 & $-2.910 \pm 1.898$ & $7.92 \mathrm{e}-4 \pm 2.78 \mathrm{e}-4$ \\
\hline$A(\mathrm{Li})$ vs. $[\mathrm{Fe} / \mathrm{H}]$ & & & \\
BA & 1.000 & $3.099 \pm 0.245$ & $0.335 \pm 0.080$ \\
ALI & 0.999 & $2.942 \pm 0.245$ & $0.258 \pm 0.085$ \\
IRFM & 0.998 & $3.047 \pm 0.268$ & $0.291 \pm 0.092$ \\
3D & 0.999 & $2.948 \pm 0.248$ & $0.274 \pm 0.083$ \\
\hline
\end{tabular}

Notes. The star CS 22188-0033 has been excluded from the fit.

that emerges. Table 8 provides the results of Kendall's $\tau$-test and the slopes of linear fits to the $A(\mathrm{Li})-[\mathrm{Fe} / \mathrm{H}]$ and $A(\mathrm{Li})-T_{\text {eff }}$ relations. The linear fits were obtained taking into account errors in both variables using the fitexy routine (Press et al. 1992). The $A(\mathrm{Li})$ error was assumed to be fixed at $0.09 \mathrm{dex}$, the error in $[\mathrm{Fe} / \mathrm{H}]$ to be given by the $\mathrm{Fe}$ I line-to-line scatter for each star, and the error in $T_{\text {eff }}$ to have a constant value of $130 \mathrm{~K}$. The sample consists of 27 of the 28 stars for which we have Li measurements, excluding CS 22882-027, for which we only have an upper limit to $A(\mathrm{Li})$, as well as CS 21188-033, where the residuals from the best-fit regressions are on the order of $3-4 \sigma$.

In statistical terms, for all four temperature scales a nonparametric Kendall's $\tau$-test indicates that $A(\mathrm{Li})$ correlates with $[\mathrm{Fe} / \mathrm{H}]$ at a very high level of significance (see Table 8 ). Moreover, a linear fit to the $A(\mathrm{Li})-[\mathrm{Fe} / \mathrm{H}]$ relation on the different 


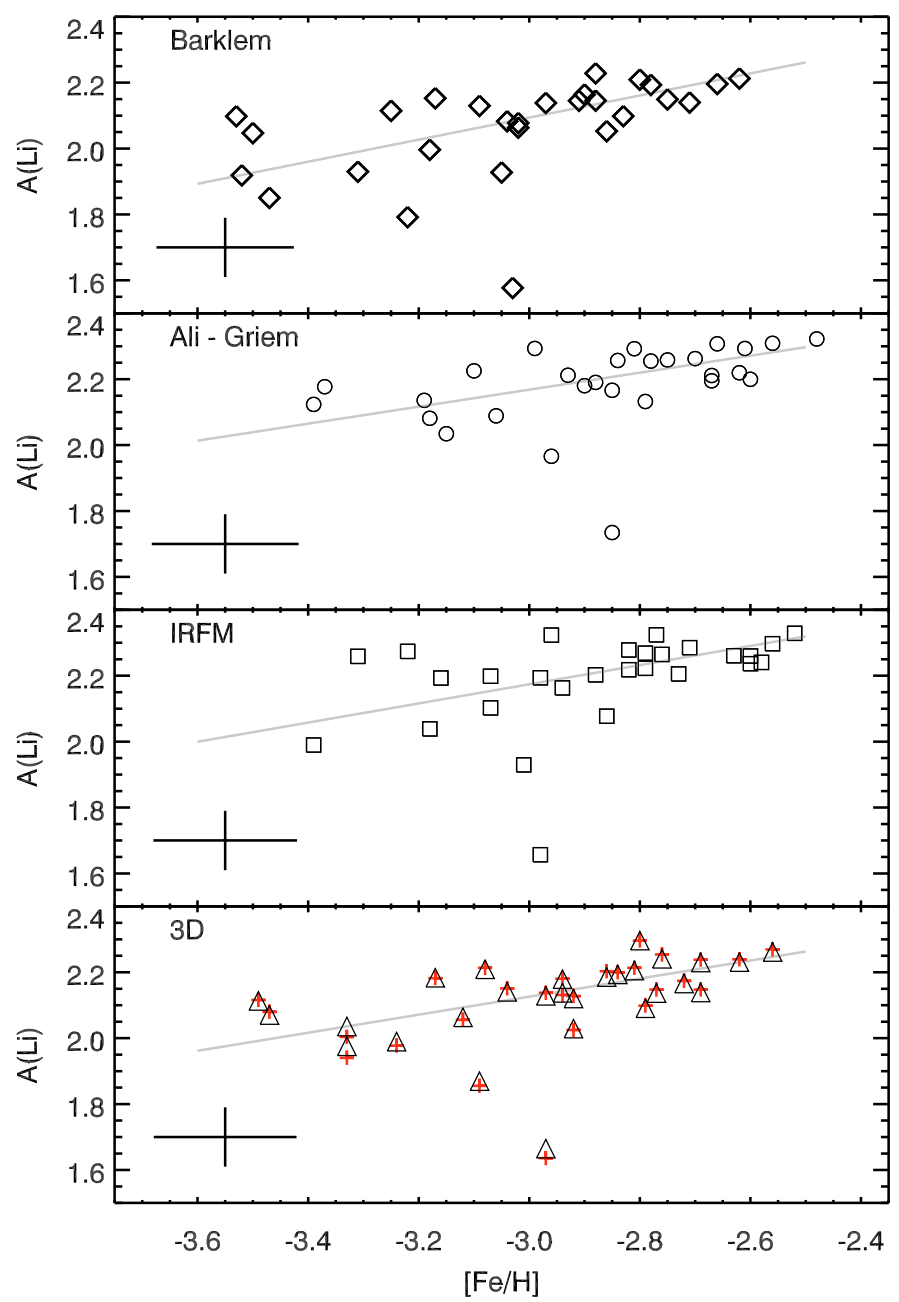

Fig. 10. $\mathrm{Li}$ abundance versus $[\mathrm{Fe} / \mathrm{H}]$ for the four temperature estimates. Top to bottom, BA, ALI, IRFM, and 3D temperatures. For the 3D temperature scale, the black triangles represent the 3D NLTE Li abundances, while the red crosses represent 1D LTE Li abundances with the NLTE corrections applied. The best-fit linear relation (as per Table 8) is indicated by a gray line. A typical error bar of \pm 0.09 dex in $\mathrm{Li}$ abundance and the average $[\mathrm{Fe} / \mathrm{H}]$ error bar are also displayed.

temperature scales produces slope values that are both always significant at the level of $3 \sigma$ and, strikingly, consistent with each other within $1 \sigma$. The slope values are also the highest reported to date. The hypothesis that the "slope" in the $A(\mathrm{Li})-[\mathrm{Fe} / \mathrm{H}]$ relation might be due to the specific $T_{\text {eff }}$ scale chosen can thus be safely rejected.

\subsection{The meltdown of the Spite plateau: slope or scatter?}

Three different $\mathrm{H} \alpha$-based $T_{\text {eff }}$ scales, as well as the totally independent IRFM scale, concur in indicating that the Spite plateau is disrupted below $[\mathrm{Fe} / \mathrm{H}] \sim-3$ (see Fig. 10). Close to that metallicity, one observes a significant increase in the Li abundance scatter, which appears to act always towards lower abundances. In other words, while some rare stars persist at the plateau level even at very low metallicity (CS 22876-032 A, González Hernández et al. 2008), the vast majority exhibit some degree of $\mathrm{Li}$ "depletion" (with respect to the plateau value).

Two stars in the sample exhibit anomalously low Li abundances. The star CS 22188-033 exhibits a mild Li depletion with

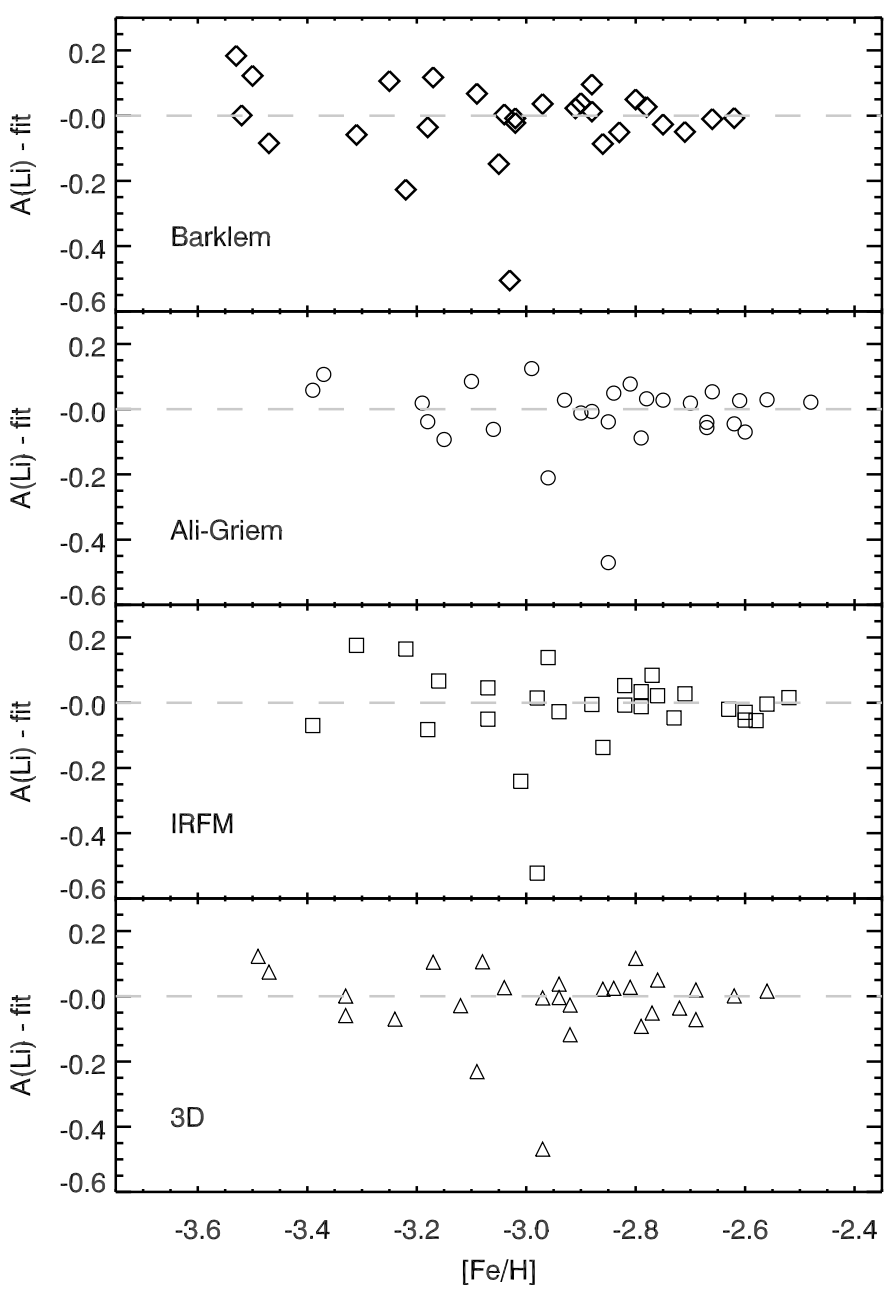

Fig. 11. Residuals of the best fit of $A(\mathrm{Li})$ vs. $[\mathrm{Fe} / \mathrm{H}]$ listed in Table 8.

a 3D NLTE $A(\mathrm{Li})=1.66$, while CS 22882-027 has no detectable Li doublet (see Sect. 7.5).

One of the much-debated results concerning the behavior of $\mathrm{Li}$ abundances in metal-poor halo dwarfs has been the reported existence of a correlation between $A(\mathrm{Li})$ and $[\mathrm{Fe} / \mathrm{H}]$, since it was first reported by Ryan et al. (1999). We investigated this by means of two different statistical tests. The Kendall's $\tau$ rankcorrelation test attempts to detect a (positive or negative) correlation, and has the fundamental strength of being non-parametric. In other words, it does not attempt to look for a specific relation to fit the data. As seen above, Kendall's $\tau$-test quite strongly supports the existence of a correlation.

The other obvious strategy we adopt is to fit the data with a linear function and see whether the slope found is statistically significant. This significance might be weakened if the data are indeed correlated, but the underlying relation is not linear. In our case, again, the slope of the linear relation is significant at $3 \sigma$ for all the considered temperature scales. This a finding, however, does not imply that the underlying "physical" relation between $[\mathrm{Fe} / \mathrm{H}]$ and $A(\mathrm{Li})$ is linear, as it would be, for example, if there was a constant Li production with increasing $[\mathrm{Fe} / \mathrm{H}]$.

To shed more light on the issue, in Fig. 11 we plot the residuals of the best-fit $A(\mathrm{Li})$ versus $[\mathrm{Fe} / \mathrm{H}]$ relation, as listed in Table 8. An increase in the scatter below $[\mathrm{Fe} / \mathrm{H}] \sim-2.8$ was already visually apparent in Fig. 10, and remains clearly recognizable in Fig. 11 once the best-fit linear relation is subtracted. To provide quantitative estimates of the level of scatter, 
we divided the sample into two in terms of metallicity, a metalricher subsample including the 8 stars with $[\mathrm{Fe} / \mathrm{H}]_{3 \mathrm{D}}>-2.8$, and a metal-poorer sub-sample including the 19 stars below that threshold. The star CS 22188-033 is plotted in the figure, but it has not been considered in this computation. We then computed the dispersion in the residuals of the two subsamples for the four temperature scales: $\sigma_{\mathrm{hi}, \mathrm{BA}}=0.04 \mathrm{dex}, \sigma_{\mathrm{lo}, \mathrm{BA}}=0.10 \mathrm{dex}$; $\sigma_{\text {hi,ALI }}=0.05 \mathrm{dex}, \sigma_{\text {lo,ALI }}=0.08 \mathrm{dex} ; \sigma_{\text {hi,IRFM }}=0.02 \mathrm{dex}$, $\sigma_{\text {lo,IRFM }}=0.10 \mathrm{dex} ; \sigma_{\text {hi,3D }}=0.05$ dex, $\sigma_{\text {lo,3D }}=0.09$ dex. For every temperature scale, the scatter in the residuals is about twice as large (or more) below $[\mathrm{Fe} / \mathrm{H}]=-2.8$ than above. It is thus clear that the tight, flat relation that is known as the Spite plateau develops both a tilt and a significant scatter at low metallicities. Once again, it is remarkable how the level of scatter appears independent of the assumed temperature scale.

We note that the true Li doublet $E W$ does not change much with metallicity, since in general $A(\mathrm{Li})$ does not vary by more than 0.2 dex. The quality of the Li doublet measurement is thus roughly constant across the whole metallicity range. The increase in scatter thus cannot be attributed to the declining quality of the measurements. On the other hand, Fe I and Fe II lines do become weaker with metallicity, which lowers the quality of the gravity estimation. Since the $\mathrm{H} \alpha$ line is quite gravity sensitive, inaccurate gravities reflect directly on $\mathrm{H} \alpha$-based $T_{\text {eff }}$ estimations, and thus on $A(\mathrm{Li})$. On the other hand, the IRFM temperature scale is totally insensitive to this effect, and yet shows the largest increase in the scatter of its residuals, and a low-metallicity scatter equal to those of the $\mathrm{H} \alpha$-based $T_{\text {eff }}$ scales. This reinforces our impression that the increase in the $A(\mathrm{Li})$ residual scatter should indeed be real.

\subsection{Plateau placement}

As a consequence of what is said above, it hardly makes sense to provide an average value for $A(\mathrm{Li})$ in our stars. One might still try, however, to determine the position of the plateau for the more metal-rich stars of the sample, which still appear to fall onto it. Every operation of this kind is somewhat arbitrary, since there is no clear-cut transition between the plateau at higher Fe content and the sloping/dispersed distribution at low metallicity. We thus decided to employ the 9 stars whose metallicity is equal or greater than -2.8 in the $3 D$ scale, and consider their $A(\mathrm{Li})$ and dispersion as being representative of the Spite plateau. The resulting values are $\langle A(\mathrm{Li})\rangle=2.164 \pm 0.059$ in the $\mathrm{BA}$ scale, $\langle A(\mathrm{Li})\rangle=2.261 \pm 0.053$ in the ALI scale, $\langle A(\mathrm{Li})\rangle=2.264 \pm$ 0.044 in the IRFM scale, and $\langle A(\mathrm{Li})\rangle=2.199 \pm 0.086$ using $A(\mathrm{Li})_{3 \mathrm{D}, \mathrm{NLTE}}$ together with the $3 \mathrm{D}$ temperature scale.

\subsection{The $A(L i)-T_{\text {eff }}$ correlation}

In Fig. 12, as well as in Table 8, a positive slope of $\mathrm{Li}$ abundance with effective temperature is present in all the temperature scales. It is, however, remarkable that this slope is driven by the coolest stars. Adopting our 3D temperature scale, if we select only the stars hotter than $6250 \mathrm{~K}$ (22 stars), the slope essentially vanishes, the probability indicated by Kendall's $\tau$ drops to $89 \%$, and a parametric test does not detect any slope.

Given the very small population of this temperature range in our sample, it is possible that we just missed any undepleted cool object. On the other hand, this might suggest that the decline (caused by convection) usually seen for stars cooler than $\sim 5700 \mathrm{~K}$ at higher metallicities may set in at higher temperatures for EMP stars (although observationally the opposite seems to be true, see Boesgaard et al. 2005).

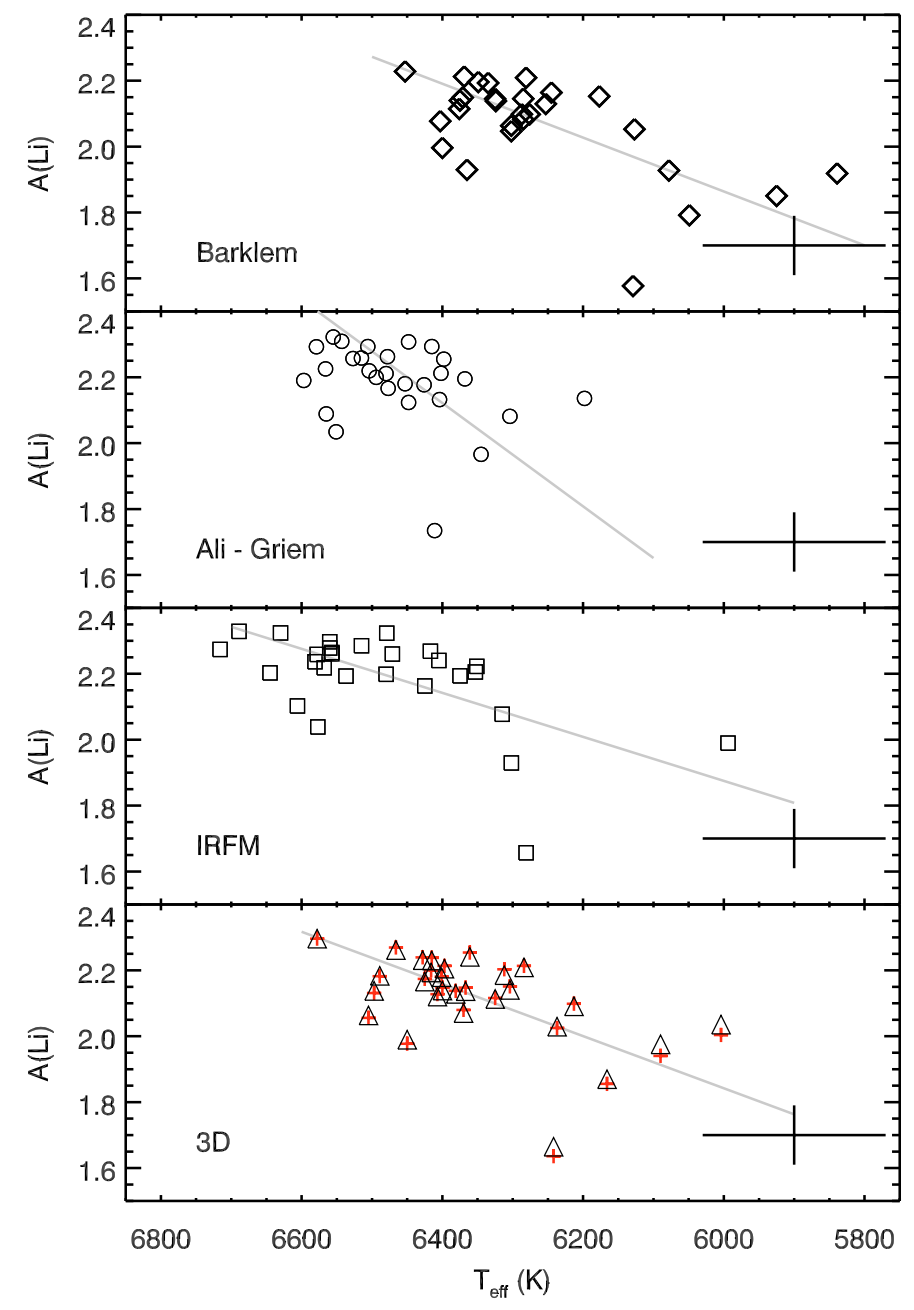

Fig. 12. Li abundance versus effective temperature for the four temperature estimates. Symbols are the same as in Fig. 10. Typical error bars of \pm 0.09 dex in the Li abundance and $\pm 130 \mathrm{~K}$ in $T_{\text {eff }}$ are also displayed. The best-fit linear relation as per Table 8 is indicated by a gray line.

We emphasize, however, that the depletion of the cool end of the sample is not driving the $A(\mathrm{Li})-[\mathrm{Fe} / \mathrm{H}]$ correlation: removing the aforementioned five cool stars has a negligible effect on this result. On the 3D scale, the Kendall's $\tau$ correlation probability of $A(\mathrm{Li})_{3 \mathrm{D}, \mathrm{NLTE}}$ with $[\mathrm{Fe} / \mathrm{H}]$ passes from 0.999 to 0.998 , while the slope of the linear fit goes from $0.274 \pm 0.083$ to $0.253 \pm 0.086$ when these 5 stars are removed. This is because the cool stars, while appearing to be all $\mathrm{Li}$ depleted, are evenly distributed in metallicity between $[\mathrm{Fe} / \mathrm{H}] \sim-3.2$ and -2.8 . On the other hand, removing these stars would affect the detected increase in the scatter at lower metallicities: applying the same residual analysis mentioned in Sect. 7.2, but now on the hot stars only, would yield $\sigma_{\text {hi,3D,hot }}=0.04$ dex (was 0.05 with the full sample), and $\sigma_{\mathrm{lo}, 3 \mathrm{D}, \text { hot }}=0.06 \mathrm{dex}$ (was 0.09 with the full sample).

\subsection{CS 22882-027 and HE 1148-0037}

Two additional stars were included in the original sample, but $\mathrm{Li}$ abundance has not been computed for them, for different reasons. We elaborate briefly on these objects.

Atmospheric parameters and metallicity were computed for CS 22882-027 (see Table 4), but the star has no detectable Li doublet (see Fig. 1). Given a $S / N \sim 80$, as measured in the Li doublet range, a typical line $F W H M$ of $0.033 \mathrm{~nm}$, and 


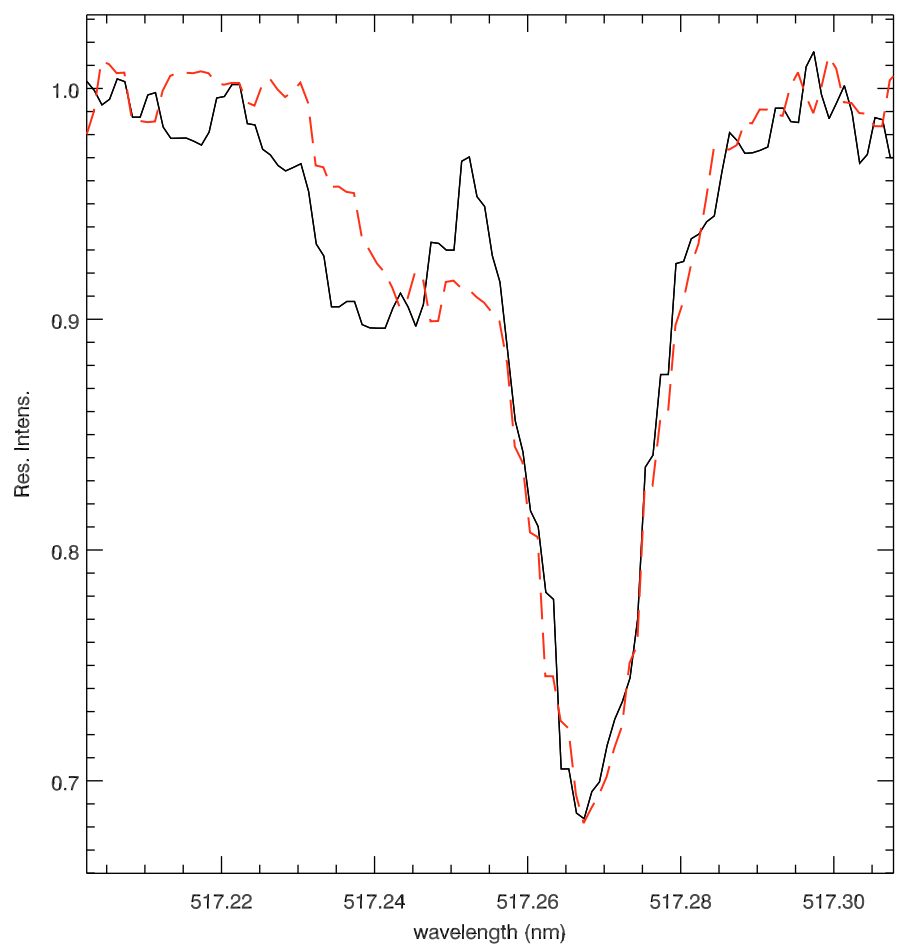

Fig. 13. The Mg I 517.268 nm line for HE 1148-0037, in spectra taken at JD 2453787.6803 (black continuous line) and JD 2453823.5443 (red dashed line), separated by 35.86 days. The spectra have been normalized, and Doppler shifted to bring the primary component to a restframe wavelength. The binarity is readily visible, as well as the variation in the components' separation.

a pixel size of $0.0027 \mathrm{~nm}$, the Cayrel (1988) formula predicts that a $\mathrm{Li}$ doublet of $E W=0.563 \mathrm{pm}$ would be measured at $3 \sigma$ confidence $(1 \sigma=0.187 \mathrm{pm})$. Employing our fuctional fit, this leads to an upper limit of $A(\mathrm{Li})_{3 \mathrm{D}, \mathrm{NLTE}} \leq 1.82$, assuming an $E W=0.563 \mathrm{pm}$ and $A(\mathrm{Li})_{3 \mathrm{D}, \mathrm{NLTE}} \leq 1.34$, assuming $E W=0.187 \mathrm{pm}$. We have a single-epoch spectrum for this star that shows no sign of a double-line system.

The star HE 1148-0037 was also originally included in the sample, but immediately set aside, since from visual inspection of the two available spectra it turned out to be a doublelined binary system. As shown in Fig. 13, the two spectra, separated by 35.86 days, clearly exhibit evidence of the double-line system, as well as readily recognizable variation in the separation between the two line systems. We were able to retrieve 3 spectra of HE 1148-0037, and measure radial velocities for the two components, which are given in Table 9, by means of cross-correlation against a synthetic template $\left(T_{\text {eff }}=6000 \mathrm{~K}\right.$, $\log g=4.0,[\mathrm{Fe} / \mathrm{H}]=-2.0)$. In our two spectra $(2006-02-21$ and 2006-03-29), cross-correlation was computed in the 490-570 nm range. We also had the lower resolution, blue-range only HERES spectrum, which was used to derive the $V_{\text {rad }}$ after masking all the broad hydrogen lines. Internal errors of the radial velocity estimate were evaluated by performing a Monte Carlo test on a sample of 50 simulated binary star spectra with noise added to emulate a $S / N=90$, the component separation and resolution being equivalent to that of the March 29, 2006 observation. The test inferred an average error of $0.072 \mathrm{~km} \mathrm{~s}^{-1}$ for the primary component and $0.246 \mathrm{~km} \mathrm{~s}^{-1}$ for the secondary. These values are representative of the internal errors in the cross-correlation procedure, but are surely dominated by the spectrograph zeropoint calibration uncertainty, which was not taken into account
Table 9. Barycentric radial velocities for the two components of the binary system HE1148-0037 as measured from the spectra available to us.

\begin{tabular}{lcccc}
\hline \hline $\begin{array}{l}\text { Date } \\
\text { UT }\end{array}$ & $\begin{array}{c}\text { Hour } \\
\text { UT }\end{array}$ & $\begin{array}{c}\text { JD } \\
\text { UT }\end{array}$ & $\begin{array}{c}V_{\text {rad }} \\
\mathrm{km} \mathrm{s}^{-1} \\
\text { primary }\end{array}$ & $\begin{array}{c}V_{\text {rad }} \\
\mathrm{km} \mathrm{s}^{-1} \\
\text { secondary }\end{array}$ \\
\hline 8 May 2003 & $11: 43: 06$ & 2452767.9882 & $-36.16 \pm 1$ & $-3.35 \pm 1$ \\
27 Feb. 2005 & $10: 33: 36$ & 2453428.94 & $-10.88 \pm 0.16$ \\
21 Feb. 2006 & $04: 19: 40$ & 2453787.6803 & $-9.15 \pm 1$ & $-22.37 \pm 1$ \\
29 Mar. 2006 & $01: 03: 46$ & 2453823.5443 & $-6.90 \pm 1$ & $-22.56 \pm 1$ \\
\hline
\end{tabular}

Notes. ${ }^{(a)}$ HERES spectrum. ${ }^{(b)}$ Aoki et al. (2009) single radial velocity.

particularly well, because very precise radial velocities were not among the goals of this study. We thus list in Table 9 an estimated total error of $1 \mathrm{~km} \mathrm{~s}^{-1}$ for all our observations, which we take to be representative of the overall systematics of these measurements.

On the other hand, Aoki et al. (2009) report AN uncertainty given by the internal scatter in the radial velocities obtained from different lines; they do not take into account the systematic uncertainties in the wavelength calibration, hence the much lower value of uncertainty. In Table 9, we kept the value they provide, but we propose that the true uncertainty is again close to $1 \mathrm{~km} \mathrm{~s}^{-1}$. If we consider their measure to be representative of the primary component radial velocity, it reproduces well the three other measurements we present.

\subsection{Comparison with other results}

Our most significant overlap is of course with the Bonifacio et al. (2007) sample, of which the present study represents a continuation. Of the 19 stars in Bonifacio et al. (2007), 17 were reanalyzed here (the two remaining stars, BS 16076-006 and BS 16968-061 turned out to be subgiants and have thus been dropped). Bonifacio et al. (2007) determined $T_{\text {eff }}$ by fitting the $\mathrm{H} \alpha$ wings fits with profiles synthesized using the Barklem et al. (2000a,b) self-broadening theory. Their temperature scale thus closely resembles our BA scale, but the $\mathrm{H} \alpha$ gravity sensitivity was not taken into account in that study $-\log g=4.0$ was assumed in computing the profiles. The effect is clearly visible in Fig. 14. The upper panel of this figure plots the temperature difference, $T_{\text {eff }}$ (this work) $-T_{\text {eff }}$ (Bonifacio et al. 2007), against the Bonifacio et al. (2007) gravity estimate. It is immediately evident that, for stars around $\log g=4.0$, the temperature difference approaches zero. For stars at higher gravities, our $T_{\text {eff }}$ estimate is below the Bonifacio et al. (2007) value by up to $200 \mathrm{~K}$. This reflects the behavior presented in Fig. 3: when a profile is fitted with a synthetic grid computed for a gravity that is underestimated, this leads to an overestimated $T_{\text {eff }}$. We note that Fig. 14 does not tell the entire story. As we do here, Bonifacio et al. (2007) estimated $\log g$ by enforcing Fe ionization equilibrium, so the derived gravity values are not identical in this work and Bonifacio et al. (2007). High-gravity and low-gravity stars do, however, retain their approximate placement in both cases, although the gravity span can be somewhat stretched by the $T_{\text {eff }}$ bias. The effect of the $T_{\text {eff }}$ difference on $[\mathrm{Fe} / \mathrm{H}]$ is shown in the middle panel of Fig. 14, while in the lower panel the difference between $A(\mathrm{Li})$ for the same stars is plotted, considering here the LTE values (to eliminate the effect of the marginally different NLTE corrections applied in the two works). The results shown are to be expected, given the strong $T_{\text {eff }}$ sensitivity of the 
L. Sbordone et al.: The metal-poor end of the Spite plateau. I.

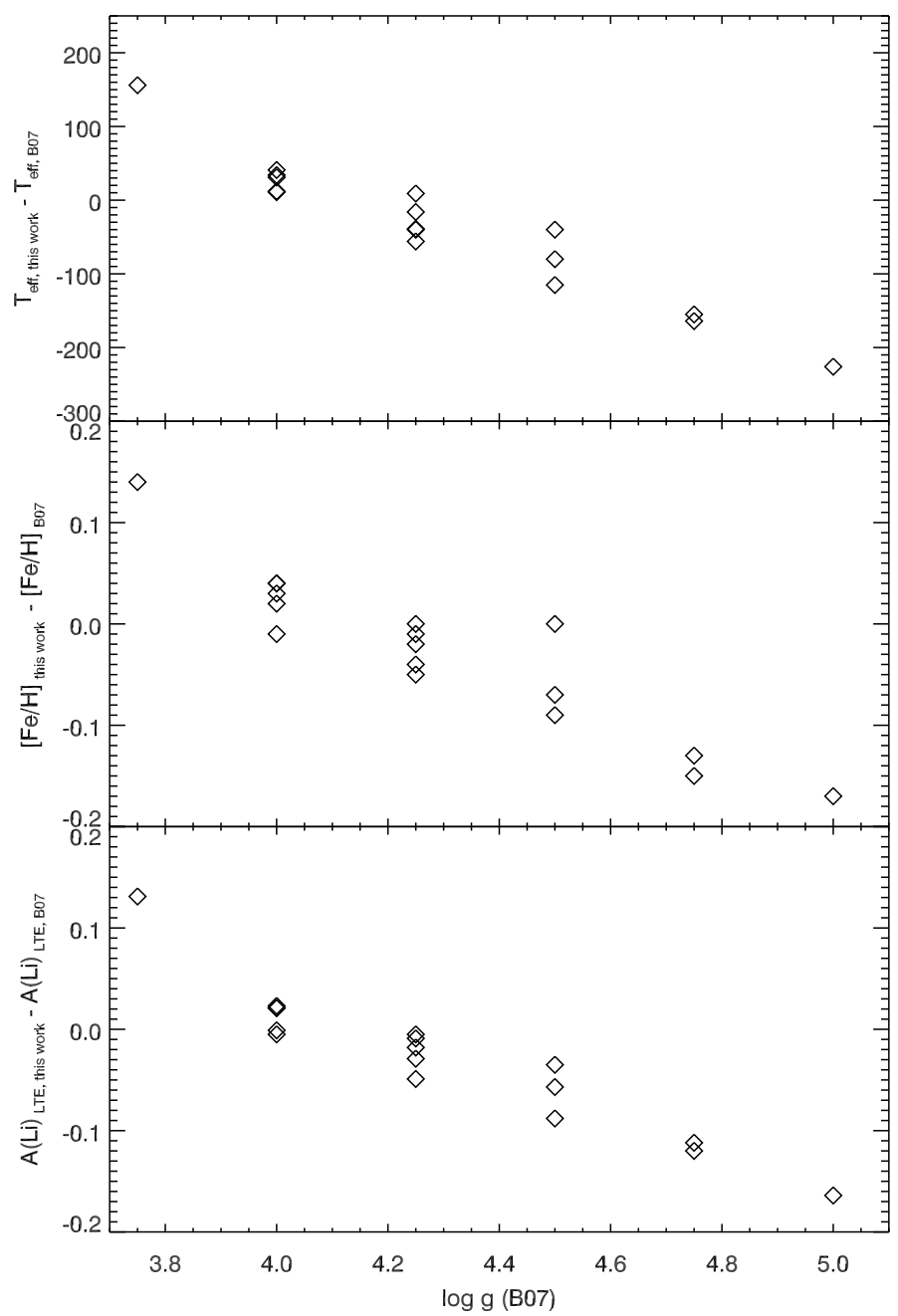

Fig. 14. Comparison between the results of this work and of Bonifacio et al. (2007) for the 17 stars in common. In the upper panel, the difference between our $T_{\text {eff }}$ and the Bonifacio et al. (2007) $T_{\text {eff }}$ determination is plotted against the value of $\log g$ in Bonifacio et al. (2007). In the center panel, we show $[\mathrm{Fe} / \mathrm{H}]$ difference, and in the lower panel, the difference in the $A(\mathrm{Li})$ LTE. For our results, BA temperature scale is used.

Li I $670.8 \mathrm{~nm}$ doublet: the current $A(\mathrm{Li})$ is higher by up to about 0.1 dex for low-gravity stars, while it is lower by roughly the same amount for high-gravity stars. On the other hand, it is easy to see how the discrepancy will only marginally affect a linear fit of $A(\mathrm{Li})$ versus $[\mathrm{Fe} / \mathrm{H}]$ : the stars are displaced roughly along a $1: 1$ diagonal in the $A(\mathrm{Li})-[\mathrm{Fe} / \mathrm{H}]$ plane.

The star LP $815-43$ is the only object that overlaps with the Asplund et al. (2006) sample. In that work, effective temperature is measured again by fitting $\mathrm{H} \alpha$ wings with a set of synthetic profiles. The details of the fitting procedure differ somewhat and the synthetic profiles are computed from MARCS models using the BSYN synthesis code. The Barklem et al. (2000a,b) selfbroadening theory is assumed here for $\mathrm{H} \alpha$, so again the BA scale is the one to be used in the comparison. The $\mathrm{H} \alpha$ gravity sensitivity is taken into account here, and the derived $T_{\text {eff }}$ is quite close to our value ( $6400 \mathrm{~K}$ vs. $6453 \mathrm{~K}$ in this work). Asplund et al. (2006) determine metallicity from Fe II lines, and gravity from Hipparcos parallaxes, but again the values do not differ much from our results $\left(\log g=4.17, V_{\text {turb }}=1.5,[\mathrm{Fe} / \mathrm{H}]=-2.74\right)$. The residual 0.14 dex offset in metallicity is in good agreement with the 0.2 dex offset detected by Bonifacio et al. (2007) between their metallicity scale and that of Asplund et al. (2006).
The lithium abundance is again quite close to our result: from the $670.8 \mathrm{~nm}$ doublet, they derive $A\left({ }^{7} \mathrm{Li}\right)=2.16$, while our value is 2.23 . The difference is fully accounted for once the $T_{\text {eff }}$ effect is considered ( $0.03 \mathrm{dex})$ as well as the already known, albeit still unexplained, 0.04 dex bias between $A(\mathrm{Li})$ as derived by means of BSYN and turbospectrum (Bonifacio et al. 2007).

The same star is also in common with Hosford et al. (2009). That work uses excitation equilibrium to estimate $T_{\text {eff }}$, a method that is not directly comparable with any of our temperature scale. The authors derive two parameter sets, one assuming the star belongs on the main sequence, and another assuming it is a subgiant. In the first case $\left(T_{\text {eff }}=6529, \log g=4.40, V_{\text {turb }}=1.4\right.$, $[\mathrm{Fe} / \mathrm{H}]=-2.61)$, they obtain a temperature that is only about $50 \mathrm{~K}$ cooler than for our $3 \mathrm{D}$ and ALI scale, but a higher gravity and metallicity. In the second case $\left(T_{\text {eff }}=6400, \log g=3.80\right.$, $\left.V_{\text {turb }}=1.4,[\mathrm{Fe} / \mathrm{H}]=-2.68\right)$, they derive a temperature $50 \mathrm{~K}$ cooler than for our BA scale, the same gravity, but again to a metallicity 0.2 dex higher. Their measured Li doublet equivalent width is about $0.2 \mathrm{pm}$ smaller than that we find, and in both cases they derive an $A(\mathrm{Li})$ that is about 0.1 dex smaller than ours. This is consistent with the expected combined effect of the difference in both $T_{\text {eff }}$ and the Li doublet $E W$.

Three stars are in common with the Aoki et al. (2009) sample, but we determined $A(\mathrm{Li})$ for only two of them, CS 22948093 and CS 22965-054. Aoki et al. (2009) employ H $\alpha$ - as well as $\mathrm{H} \beta$-wing fitting to determine $T_{\text {eff }}$ on the basis of MARCS models and using the Barklem et al. (2000a,b) self-broadening for $\mathrm{H} \alpha$. Once more, our BA temperature scale is the one most appropriate for a comparison. The $\mathrm{H} \alpha$ gravity sensitivity is taken into account. Surface gravities are estimated by comparing with isochrones as well as by evaluating the Fe I-Fe II ionization equilibrium. The parameter values they derive for CS 22948-093 are in close agreement with those we derive $\left(T_{\mathrm{eff}}(\mathrm{H} \alpha)=6320 \mathrm{~K}\right.$, $\left.T_{\text {eff }}=6380, \log g=4.4, V_{\text {turb }}=1.5\right)$, while $[\mathrm{Fe} / \mathrm{H}]$ is $0.13 \mathrm{dex}$ lower at -3.43 . The derived value of $A(\mathrm{Li})_{\mathrm{LTE}}=1.96$ is inexcellent agreement with our value of 1.935. CS 22965-054 shows a more significant discrepancy in $T_{\text {eff }}$. Their value of $T_{\text {eff }}(\mathrm{H} \alpha)=$ $6390 \mathrm{~K}$ is remarkably higher than our, while $T_{\text {eff }}(\mathrm{H} \beta)$ is much closer to our value. Since the adopted temperature is the average of the two, the final $T_{\text {eff }}$ is ultimately just $56 \mathrm{~K}$ hotter than our value. Their derived surface gravity is also very close in value $(\log g=3.9)$, while $V_{\text {turb }}$ is the same. However, Aoki et al. (2009) derive the same lithium abundance as we do, $A(\mathrm{Li})_{\mathrm{LTE}}=2.16$, due to their lower measured value of $E W(2.03 \mathrm{pm})$ for the Li doublet.

The third star in common with Aoki et al. (2009) is HE 1148-0037, for which Aoki et al. (2009) do not appear to have noticed its binarity. On close inspection, the single spectrum they employed (JD 2453 428.94) shows signs of line asymmetry (Aoki 2009, priv. comm.), but it appears to have been taken quite close to conjunction. In contrast, as seen in Sect. 7.5, the three spectra we have of this star all show quite clearly the two line systems.

We observed No star in common with Meléndez \& Ramírez (2004). A comparison with their sample is nevertheless interesting due to the extension of their sample to very low metallicities, despite with a limited number of stars (a total of 10 stars were analyzed below $[\mathrm{Fe} / \mathrm{H}]=-2.5,4$ at or below $[\mathrm{Fe} / \mathrm{H}]=$ -3). Meléndez \& Ramírez (2004) detected no slope in the Spite plateau, for which they advocated a high value of $\langle A(\mathrm{Li})\rangle=2.37$. As noticed in González Hernández \& Bonifacio (2009), the $T_{\text {eff }}$ scale adopted by Meléndez \& Ramírez (2004) is systematically hotter than the one we employ for metal poor dwarfs, on average by $87 \mathrm{~K}$. This explains half of the descrepancy between our 


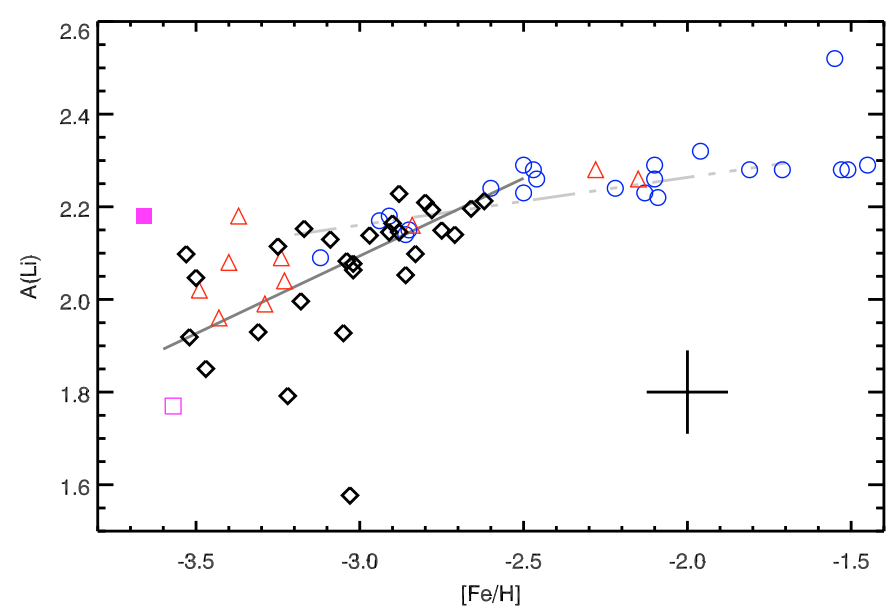

Fig. 15. A unified view of $A(\mathrm{Li})$ vs. $[\mathrm{Fe} / \mathrm{H}]$ from some studies for which a common temperature scale can be assumed. Blue circles, Asplund et al. (2006) data, red triangles, Aoki et al. (2009) data, magenta squares, CS 22876-032 from González Hernández et al. (2008), filled symbol primary star, open symbol secondary star. Black diamonds, this work, BA temperature scale. Dot-dashed gray line, best linear fit to Asplund et al. (2006) data, continuous dark gray line, best fit to our data. Typical error bars for our data are displayed.

average IRFM plateau placement and their own, and can account in principle for their failure to detect the slope, assuming their temperature scale and ours diverge progressively at low metallicities.

Lithium abundances for two extremely metal poor stars (HE 0233-0343 and HE 0945-1435) were recently presented by García Pérez et al. (2008). Both stars show extremely low Fe content $([\mathrm{Fe} / \mathrm{H}] \sim-4)$, but probably because of the weakness of Fe II lines, the estimation of gravity is uncertain. This affects the determinations of both the evolutionary status (either MS or early SGB) and $T_{\text {eff }}$, which is derived from $\mathrm{H} \alpha$ wing fitting in a way similar to that used with our BA scale. The stars appear to be fairly cool, $6000 \mathrm{~K} \leq T_{\text {eff }} \leq 6250 \mathrm{~K}$, which would place them among the "cool stars" of our sample as described in Sect. 7.4, and both objects show significantly depleted Li, $A(\mathrm{Li}) \sim 1.8$. Owing to the uncertainty of the parameters determination we did not include these stars in Fig. 15.

\section{Possible biases}

\subsection{Binary stars}

Two potential biases can, in principle, be responsible for producing systematically low $\mathrm{Li}$ abundances, and a trend of $A(\mathrm{Li})$ with metallicity. The first one is of course the presence of undetected binaries, for which the veiling by the secondary star will systematically reduce the $E W$ of the lines of the primary, leading to an underestimate of both metallicity and Li abundance. The true impact of this effect is difficult to evaluate, mainly because little is known about the fraction, and mass-ratio distribution, of binaries at low metallicities. Duquennoy et al. (1991) report, for $\mathrm{G}$ dwarfs in the solar vicinity, a fraction of $44 \%$ of stars having a companion with $q=M_{2} / M_{1}>0.1$, about $1 / 3$ of which have $q>0.5$. Latham et al. (2002, and references therein) found that the halo binary population does not differ significantly from the disk one, although we note that they did not explore significant numbers of stars with metallicities as low as the stars in our present sample.
It nevertheless seems unlikely that undetected binaries pollute our sample significantly. We checked for binarity by inspecting the $\mathrm{Mg}$ b triplet lines (e.g., see Fig. 13). Our spectra have a typical $S / N \sim 100$ or higher, and $\mathrm{Mg}$ b lines have a typical $E W$ of $10 \mathrm{pm}$. A line with central residual intensity of 0.95 would be detected at least at the $5 \sigma$ level in this typical spectrum, and have a typical $E W$ of $0.8 \mathrm{pm}$. As per González Hernández et al. (2008), to reduce a $10 \mathrm{pm}$ line to $0.8 \mathrm{pm}$, a ratio of the continua fluxes of about 11.5 is needed. If we roughly assume that the total luminosity scales accordingly, this corresponds roughly to $q=0.5$ (since $L \propto M^{3.2}$ on the main sequence, see Kippenhahn $\&$ Weigert 1990). A similar flux ratio in the Li doublet range would lead to a correction of the Li doublet $E W$ for the primary star of about $8 \%$, corresponding to 0.03 dex in $A(\mathrm{Li})$. In other words, every binary star requiring significant veiling correction on the primary spectrum would also be promptly detectable because of the double line system. This system could only stay undetected if the radial velocity separation of the two stars was quite small at the moment of the observation(s), so that the two line systems remained blended.

In addition to the above, if we take the figures of Duquennoy et al. (1991) at face value, find that about $13.5 \%$ of the binaries are characterized by a significant veiling of the primary (i.e. $q>0.5$ ). Our original sample comprised 30 stars, which implies that there are 4 expected "significant binaries". Three stars have already been rejected from the sample, one of them (HE 1148-0037) being indeed a binary. Two more (CS 22882027 and CS 22188-033) exhibit significant lithium depletion or no Li doublet at all, and were excluded from all statistical analyses. They are clearly the most likely candidates to be binaries, albeit neither one shows a double line system ${ }^{7}$. One could thus expect one more "disguised" binary to be biasing the sample. While this is quite possible, it would hardly influence any of our results.

\subsection{D NLTE effects on Fe ionization equilibrium}

The second problem relates to the use of Fe I-Fe II ionization equilibrium to estimate gravity. From preliminary computations, it appears that $3 \mathrm{D}$ corrections of $\mathrm{Fe}$ lines with excitation potentials of the same order as employed in the present work could be quite large at low metallicities for stars similar to those that we study. Moreover, corrections for Fe I appear to be negative (of about $0.2 \mathrm{dex}$ ), while they are positive (about $0.1 \mathrm{dex}$ ) for Fe II lines, for a $T_{\text {eff }}=6500, \log g=4.5,[\mathrm{Fe} / \mathrm{H}]=-3.0$ star. The phenomenon is mainly caused by the overcooling that $3 \mathrm{D}$ treatment produces in the outer layers of atmospheres at low metallicities, and appears to be of similar magnitude at $[\mathrm{Fe} / \mathrm{H}]=-2$ (due to the stronger saturation of $\mathrm{Fe}$ lines, which drives their contribution function to higher layers), but would most likely disappear above. If taken at face value, a 0.3 dex Fe I-Fe II imbalance would lead to an overestimate of $\log g$ of about 0.5 dex when analyzed using 1D LTE models (as is our case regarding metallicity and gravity estimation). We do indeed find higher gravities than expected from evolutionary tracks. On the other hand, we do not currently have a 3D NLTE spectrosynthesis code for iron; we are thus unable to account for NLTE effects, which are likely to counterbalance the $3 \mathrm{D}$ effect because of overionization occurring in the upper layers where overcooling is present in 3D models. A similar mechanism is indeed active for

\footnotetext{
7 We have another UVES spectrum of CS 22188-033, taken at a different epoch, which does not show radial velocity variations with respect to the one used in the present work, nor signs of a double line system.
} 
$\mathrm{Li}$, whose 3D LTE abundance derived by $\mathrm{CO}^{5}$ BOLD-LINFOR3D is about 0.2 dex below the corresponding 1D NLTE value, while the 3D NLTE one is essentially indistinguishable from the 1D NLTE result (Figs. 10 and 12). If some degree of imbalance remains after NLTE is taken into account, and is metallicity sensitive, this might introduce a bias when $T_{\text {eff }}$ is determined by $\mathrm{H} \alpha$-wing fitting. It is indeed intriguing to note how lower gravity estimates lead to higher temperatures, and as a consequence, (somewhat) higher the $\mathrm{Li}$ abundances. On the other hand, the IRFM temperature scale should be immune to this problem, $A$ (Li) being quite insensitive to $\log g$ itself, and the IRFM-based analysis inferring abundances similar to the $\mathrm{H} \alpha$-based estimate.

\section{Conclusions}

We have presented the largest sample to date of Li abundances for EMP halo dwarf stars (27 abundances and one upper limit), including the largest sample to date below $[\mathrm{Fe} / \mathrm{H}]=-3(10$ abundances). Lithium abundance determination is highly sensitive to biases in the effective temperature scale, and we have tried to account for this using four different temperature estimators. In an additional effort to accurately represent the stellar atmospheres of the sample stars, 3D, time-dependent, hydrodynamical atmosphere models have been used to determinine our preferred $\mathrm{H} \alpha$-based temperature scale, and a detailed 3D NLTE spectrosynthesis has been applied to the determination of lithium abundance. Both these techniques have been employed here for the first time, to our knowledge, in the analysis of EMP stars. This has also allowed us to develop a useful fitting formula allowing one to derive $A(\mathrm{Li})_{3 \mathrm{D}, \mathrm{NLTE}}$ directly as a function of $E W$, $T_{\text {eff }}, \log g$, and $[\mathrm{Fe} / \mathrm{H}]$ for EMP turn-off and early subgiant stars (see Appendix B).

The first obvious conclusion of this work is that we have confirmed what was merely suggested by the analysis of Bonifacio et al. (2007), and previous works, that at the lowest metallicity there is sizable dispersion in the $\mathrm{Li}$ abundances and that there is a trend of decreasing Li abundance with decreasing metallicity. We have also shown that these two conclusions do not depend on the adopted temperature scale, as suggested by Molaro (2008). The results hold, qualitatively, using both IRFM temperatures and $\mathrm{H} \alpha$ temperatures, regardless of the broadening theory adopted and irrespective of the use of either 1D or 3D model atmospheres. Quantitatively, the results differ in the mean level of the $\mathrm{Li}$ abundance, while the slopes in the $A(\mathrm{Li})$ versus $[\mathrm{Fe} / \mathrm{H}]$ relations agree within errors. None of the temperature scales investigated produces a "flat" Spite plateau over the full range in $[\mathrm{Fe} / \mathrm{H}]$ (see Table 8 ).

Our results are in substantial agreement with those of Aoki et al. (2009). While these authors do not detect a slope with either effective temperature or metallicity, this happens simply because of the small extent of their sample in both these parameters. On the other hand, they do point out that their sample has a lower Li abundance than that observed at higher metallicities.

The picture outlined by the aforementioned results acquires more significance, once we place it in a broader context among the latest studies regarding lithium in EMP stars. Figure 15 compares our results with those of three investigations employing compatible temperature scales. In this figure, open blue circles represent stars from the Asplund et al. (2006) sample, red triangles from the Aoki et al. (2009) data, and the two magenta squares the two components of the double-lined binary system CS 22876-032 (González Hernández et al. 2008, the filled square corresponds to the primary star). Our data are represented as black diamonds (the results of the BA scale are shown, for compatibility with the temperature scales used in the other three works $)^{8}$. The best linear fit to our data is shown as a dark gray solid line, while the best fit to Asplund et al. (2006) data $(A(\mathrm{Li})=2.409+0.103[\mathrm{Fe} / \mathrm{H}])$ is shown by a dot-dashed gray line. The Asplund et al. (2006) Li abundances are increased here by 0.04 dex to account for the known offset already mentioned in Sect. 7.6, and their metallicty is decreased by 0.2 dex to correspond to the metallicity-scale offset detected by Bonifacio et al. (2007). It is now even more evident that the Spite plateau does not exist anymore at the lowest metallicity, and is replaced by an increased spread of abundances, apparently covering a roughly triangular region ending quite sharply at the plateau level. This region appears here to be populated in a remarkably even manner; at any probed metallicity some star remains at, or very close to, the Spite plateau level, but many do not. The rather different slopes of the best-fit relations in Asplund et al. (2006) and in this work appear to be the obvious consequence of fitting two subsamples covering different metallicity regimes. This could provide also an explanation for the numerous claims, starting from Ryan et al. (1999), of a thin, but tilted Spite plateau. From this view, the difference was produced simply because the tail of these samples had been falling in the low-metallicity "overdepletion zone" as we have been able to discern more clearly.

We are not aware of any theoretical explanation of this behavior. After the measurements of the fluctuations of the CMB made it clear that there is a "cosmological lithium problem", i.e., the Li predicted by SBBN and the measured baryonic density is too high with respect to the Spite plateau (by about 0.6 dex for our sample), there have been many theoretical attempts to provide Li-depletion mechanisms that would reduce the primordial Li to the Spite plateau value in a uniform way. Our observations now place anadditional constraint on these models - below a metallicity of about $[\mathrm{Fe} / \mathrm{H}]=-2.5$, they should cause a dispersion in $\mathrm{Li}$ abundances and an overall lowering of $A(\mathrm{Li})$.

If $\mathrm{Li}$ depletion from the WMAP-prescribed level were to happen in the stellar envelopes of very metal-poor stars, the mechanism would have to be remarkably metallicity insensitive to account for the thin, flat plateau observed between $[\mathrm{Fe} / \mathrm{H}]=$ -2.5 and -1 . And yet, the same phenomenon must become sharply metallicity sensitive around and below $[\mathrm{Fe} / \mathrm{H}]=-2.5$, i.e., precisely where metallicity effects on the atmospheric structure are expected to become vanishing small.

We are tempted to imagine that two different mechanisms may need to be invoked to explain the production of the Spite plateau for stars with $[\mathrm{Fe} / \mathrm{H}]>-2.5$, and of the low-metallicity dispersion for stars with $[\mathrm{Fe} / \mathrm{H}]<-2.5$. One could envision such a two-step process as follows:

1. Metal-poor halo stars are always formed at the Spite plateau level, regardless of their metallicity. Whether the plateau represents the cosmological $\mathrm{Li}$ abundance or is the result of some primordial uniform depletion taking place before the star formation phase is immaterial in this context.

2. A second phenomenon, possibly related to atmospheric diffusion, becomes active around $[\mathrm{Fe} / \mathrm{H}]=-2.5$ and below, depleting Li further in the atmosphere of EMP stars. This phenomenon, aside from the metallicity sensitivity, would exhibit different star-to-star efficiency, being possibly dependent on additional parameters, such as stellar rotation or $T_{\text {eff }}$. Its efficiency must in any case be higher for more metal-poor stars.

${ }^{8}$ González Hernández et al. (2008) derived $T_{\text {eff }}$ from photometry and isochrones, but a cross-check with $\mathrm{H} \alpha$ profiles computed in 1D with Barklem et al. (2000a) broadening confirmed the result. 
In this scenario, the "primordial" plateau would be preserved above $[\mathrm{Fe} / \mathrm{H}] \sim-2.5$, but below that metallicity, a systematic "leakage" of stars towards lower $A(\mathrm{Li})$ would take place, more effectively for more metal-poor stars, but naturally scattered due to the sensitivity to parameters other than $[\mathrm{Fe} / \mathrm{H}]$. This scheme would have a number of advantages. First of all, it would naturally explain our observations, "mimicking" a slope in $A(\mathrm{Li})$ versus $[\mathrm{Fe} / \mathrm{H}]$, but with increased scatter at low $[\mathrm{Fe} / \mathrm{H}]$. It would also explain why, while the scatter in $A(\mathrm{Li})$ increases at low metallicities, not a single star in this metallicity regime has been found to lie above the Spite plateau level. It would then be consistent with a small number of stars remaining close to the plateau at any metallicity (e.g., CS 22876-032 A, González Hernández et al. 2008, filled magenta square in Fig. 15); in these objects, the depletion process would be somehow inhibited. Finally, attributing the extra depletion to atmospheric diffusion/settling would not require a physical "conspiracy" capable of producing exactly the same depletion level regardless of metallicity, stellar rotation, gravity, or effective temperature, as is often invoked when diffusion is used to explain the Spite plateau.

The nature of what we refer to above as the "second phenomenon", the one responsible for the departures from the Spite plateau below $[\mathrm{Fe} / \mathrm{H}]=-2.5$, is perhaps the most intriguing. Above, we have proposed some kind of photospheric settling mechanism, but one could as well envision a chemical evolution scenario, on the basis of some gas pre-processing with Li depletion (à la Piau et al. 2006) - while it may not be able to account for the entire WMAP-Spite plateau discrepancy, this mechanism could easily account for the mild (0.2-0.4 dex) departure from the plateau observed at lower metallicities. Moreover, this mechanism would naturally produce a spread of abundances as a consequence of the local level of gas pre-processing.

There are hints that the recently discovered ultra-faint dwarf galaxies (uFdg) might have been the source of the bulk of the EMP stars now found in the halo of the Milky Way (Tolstoy et al. 2009, and references therein). If this were indeed the case, a sizeable fraction of our sample could have formed in uFdg systems, possibly more so for the most metal-poor objects. It has been suggested (Komiya et al. 2009) that the paucity of stars below $[\mathrm{Fe} / \mathrm{H}]=-3.5$ may be due to the onset of self-pollution in the primordial mini-halos when they started to merge to form larger structures, and ultimately the halo. One could then envision that gas reprocessing could have fairly significantly altered the Li abundances in the heavily dark-matter dominated cores of these sub-halos, but become progressively negligible when they merged, and their ISM mixed more and more completely with gas of pristine Li abundance. Sub-plateau stars may then originate from star formation that occurred in the sub-halo cores, or while mixing with pristine gas that progressively diluted the effect of the reprocessing.

In any case, the main drawback of this two-phenomena scenario is to leave the WMAP-Spite plateau discrepancy unexplained. It also implies that any depletion from WMAP-based primordial $A(\mathrm{Li})$ should have taken place before the currently observed stars formed. Non-standard primordial nucleosynthesis thus remains entirely viable, until the contrary is proven.

Looking retrospectively at the history of the abundances of light elements and SBBN, one concludes that if the Spite plateau is the result of significant uniform Li depletion among metalpoor stars, one faces a formidable case of cosmic conspiracy. It is necessary to admit that the Li has been depleted quite precisely to a level consistent with SBBN production and also consistent with the abundances of the other measurable light elements. Had Li been depleted to the level of $A(\mathrm{Li})=1.8$, that is, below the minimum allowed by SBBN, the Spite plateau might not have been interpreted as being related to primordial Li, right from the beginning.

A caveat is in order when looking at Fig. 15: the Asplund et al. (2006) sample is biased, having been purposedly selected not to include objects significantly deviating from the Spite plateau. In fact, stars with varying degrees of Li depletion have long been known to exist (e.g., Charbonnel \& Primas 2005). On the other hand, warm (i.e., $T_{\text {eff }}>6000 \mathrm{~K}$ ) dwarf stars deviating from the Spite Plateau appear to be quite rare at $[\mathrm{Fe} / \mathrm{H}]>$ -3 . Again, our sample is limited towards higher metallicities, which prevents us from properly quantifying this statement. A re-evaluation of the $\mathrm{Li}$ abundances above $[\mathrm{Fe} / \mathrm{H}]=-2.5$ is in order, using an analysis technique homogeneous with the present one, and applied to a sample for which a well-known selection technique has been applied. It is plain to see that, had previous authors encountered $\mathrm{Li}$ abundance distributions as the one observed by us and Aoki et al. (2009), the concept of a lithium abundance plateau would have been short lived. Instead, there is a clear perception that mildly Li depleted stars do exist among MP stars, but are a rare occurrence. We cannot easily identify any reason why all the previous studies should have been biased in a different way with respect to the present one, but until a coherent work, based on a well defined selection criterion, is extended to higher metallicities, all we are left with is just this perception.

Two other lines of future investigation are suggested by the present results: $(i)$ increase the sample of hot $\left(T_{\text {eff }} \geq 6250 \mathrm{~K}\right)$ ) EMP stars $([\mathrm{Fe} / \mathrm{H}] \leq-3.0)$, and (ii) increase the number of cool $\left(T_{\text {eff }}<6250 \mathrm{~K}\right)$ stars over the entire metallicity range. The present sample has seven hot EMP stars, five of which have $A(\mathrm{Li})$ below the Spite plateau. Even if "standard" ZAMS $\mathrm{Li}$ depletion was setting in at higher than usual temperatures in EMP stars, these objects are unlikely to be affected by it. Statistics is still weak in this metallicity regime, and that the primary component of CS 22876-032 lies at the level of the Spite plateau, at a metallicity of $[\mathrm{Fe} / \mathrm{H}]=-3.6$, clearly calls for a search for similar objects. At the same time, the cool end of our sample is presently too poorly populated to draw any definitive conclusion about whether these stars are experiencing convective depletion, which again, calls for an enlargement of the cool stars sample at low $([\mathrm{Fe} / \mathrm{H}]<-2.5)$ metallicities.

To help understand the phenomena involved (since there could be several at work) that bring about the observed Li abundance pattern, another important issue is to assign an accurate evolutionary status to each star, that is, to confidently assess which stars are dwarfs, main-sequence turnoff, or subgiants. Our surface gravities are not sufficiently accurate for this purpose. The GAIA satellite will provide accurate parallaxes for all of the presently studied stars, thus allowing one to more clearly elucidate this problem.

Acknowledgements. Authors L.S., P.B., H.-G.L., N.B., and J.G.-H. acknowledge financial support from EU contract MEXT-CT-2004-014265 (CIFIST). We acknowledge use of the supercomputing center CINECA, which has granted us time to compute part of the hydrodynamical models used in this investigation, through the INAF-CINECA agreement 2006, 2007. T.C.B. acknowledges partial support for this work from grant 08-22648: Physics Frontier Center/Joint Institute for Nuclear Astrophysics. This research has made use of the SIMBAD database, operated at CDS, Strasbourg, France, and of NASA's Astrophysics Data System. This publication makes use of data products from the Two Micron All Sky Survey, which is a joint project of the University of Massachusetts and the Infrared Processing and Analysis Center/California Institute of Technology, funded by the National Aeronautics and Space Administration and the National Science Foundation. We also wish to thank K. Lind for making available to us her code to interpolate the NLTE correction tables of Lind et al. (2009a). 
L. Sbordone et al.: The metal-poor end of the Spite plateau. I.

\section{References}

Ali, A. W., \& Griem, H. R. 1966, Phys. Rev., 144, 366

Allard, N. F., Kielkopf, J. F., Cayrel, R., \& van't Veer-Menneret, C. 2008, A\&A, 480,581

Allen, C.W. 1976, Astrophysical Quantities, 3rd edition (The Athlone Press), 41

Alonso, A., Salaris, M., Arribas, S., Martínez-Roger, C., \& Asensio Ramos, A. 2000, A\&A, 355, 1060

Alonso, A., Arribas, S., \& Martínez-Roger, C. 2001, A\&A, 376, 1039

Alvarez, R., \& Plez, B. 1998, A\&A, 330, 1109

Aoki, W., Barklem, P. S., Beers, T. C., et al. 2009, ApJ, 698, 1803

Asplund, M., Gustafsson, B., Kiselman, D., \& Eriksson, K. 1997, A\&A, 318, 521

Asplund, M., Grevesse, N., \& Sauval, A. J. 2005, Cosmic Abundances as Records of Stellar Evolution and Nucleosynthesis, ASP Conf. Ser., 336, 25

Asplund, M., Lambert, D. L., Nissen, P. E., Primas, F., \& Smith, V. V. 2006, ApJ, 644, 229

Barklem, P. S., Piskunov, N., \& O’Mara, B. J. 2000a, A\&A, 355, L5

Barklem, P. S., Piskunov, N., \& O’Mara, B. J. 2000b, A\&A, 363, 1091

Barklem, P. S., Stempels, H. C., Allende Prieto, C., et al. 2002, A\&A, 385, 951

Barklem, P. S., Belyaev, A. K., \& Asplund, M. 2003, A\&A, 409, L1

Barklem, P. S., Christlieb, N., Beers, T. C., et al. 2005, A\&A, 439, 129

Beers, T. C. 1999, Ap\&SS, 265, 547

Beers, T. C., Preston, G. W., \& Shectman, S. A. 1985, AJ, 90, 2089

Beers, T. C., Preston, G. W., \& Shectman, S. A. 1992, AJ, 103, 1987

Behara, N. T., Ludwig, H.-G., Steffen, M., \& Bonifacio, P. 2009, AIP Conf. Ser. 1094, 784

Blackwell, D. E., \& Shallis, M. J. 1977, MNRAS, 180, 177

Blackwell, D. E., Petford, A. D., \& Shallis, M. J. 1980, A\&A, 82, 249

Blackwell, D. E., Petford, A. D., Arribas, S., Haddock, D. J., \& Selby, M. J. 1990, A\&A, 232, 396

Boesgaard, A. M., Stephens, A., \& Deliyannis, C. P. 2005, ApJ, 633, 398

Bonifacio, P., \& Molaro, P. 1997, MNRAS, 285, 847

Bonifacio, P., Monai, S., \& Beers, T. C. 2000, AJ, 120, 2065

Bonifacio, P., Molaro, P., Sivarani, T., et al. 2007, A\&A, 462, 851

Caffau, E., \& Ludwig, H.-G. 2007, A\&A, 467, L11

Caffau, E., Steffen, M., Sbordone, L., Ludwig, H.-G., \& Bonifacio, P. 2007, A\&A, 473, L9

Carretta, E., Gratton, R., Cohen, J. G., Beers, T. C., \& Christlieb, N. 2002, AJ, 124,481

Carlsson, M., Rutten, R. J., Bruls, J. H. M. J., \& Shchukina, N. G. 1994, A\&A, 288,860

Castelli, F., \& Kurucz, R. L. 2003, Modelling of Stellar Atmospheres, 210, 20P, [arXiv:astro-ph/0405087]

Cayrel, R. 1988, in The Impact of Very High $S / N$ Spectroscopy on Stellar Physics, ed. G. Cayrel de Strobel, \& M. Spite (Dordrecht: Kluwer), 345

Cayrel, R., Steffen, M., Chand, H., et al. 2007, A\&A, 473, L37

Charbonnel, C., \& Primas, F. 2005, A\&A, 442, 961

Christlieb, N., Schörck, T., Frebel, A., et al. 2008, A\&A, 484, 721

Cohen, J. G., Christlieb, N., Beers, T. C., Gratton, R., \& Carretta, E. 2002, AJ 124,470

Cox, A. N. 2000, Allen's Astrophysical Quantities, 4rd edition, ed. A. N. Cox, AIP (Springer), 40

Cyburt, R. H., Fields, B. D., \& Olive, K. A. 2008, J. Cosmol. Astro-Part. Phys., 11,12

Dekker, H., D’Odorico, S., Kaufer, A., Delabre, B., \& Kotzlowski, H. 2000, Proc. SPIE, 4008, 534

Dunkley, J., Komatsu, E., Nolta, M. R., et al. 2009, ApJS, 180, 306

Duquennoy, A., Mayor, M., \& Halbwachs, J.-L. 1991, A\&AS, 88, 281

Edvardsson, B., Andersen, J., Gustafsson, B., et al. 1993, A\&A, 275, 101

Frebel, A., Collet, R., Eriksson, K., Christlieb, N., \& Aoki, W. 2008, ApJ, 684, 588
Freytag, B., Steffen, M., \& Dorch, B. 2002, Astron. Nachr., 323, 213

Fuhrmann, K., Axer, M., \& Gehren, T. 1993, A\&A, 271, 451

García Pérez, A. E., Christlieb, N., Ryan, S. G., et al. 2008, Physica Scripta V. T, 133,014036

González Hernández, J. I., \& Bonifacio, P. 2009, A\&A, 497, 497

González Hernández, J. I., Bonifacio, P., Ludwig, H.-G., et al. 2008, A\&A, 480, 233

Grevesse, N., \& Sauval, A. J. 1998, Space Sci. Rev., 85, 161

Gustafsson, B., Bell, R. A., Eriksson, K., \& Nordlund, A. 1975, A\&A, 42, 407

Gustafsson, B., Edvardsson, B., Eriksson, K., et al. 2003, Stellar Atmosphere Modeling, 288, 331

Hosford, A., Ryan, S. G., García Pérez, A. E., Norris, J. E., \& Olive, K. A. 2009, A\&A, 493, 601

Iocco, F., Mangano, G., Miele, G., Pisanti, O., \& Serpico, P. D. 2009, Phys. Rep., 472,1

Kippenhahn, R., \& Weigert, A. 1990, Stellar Structure and Evolution (Berlin, Heidelberg, New York: Springer-Verlag), XVI, 468, also Astronomy and Astrophysics Library

Komiya, Y., Suda, T., \& Fujimoto, M. Y. 2009, ApJ, 694, 1577

Korn, A. J., Grundahl, F., Richard, O., et al. 2006, Nature, 442, 657

Korn, A. J., Grundahl, F., Richard, O., et al. 2007, ApJ, 671, 402

Kurucz, R. L. 2005, Mem. Soc. Astron. Ital. Suppl., 8, 14

Latham, D. W., Stefanik, R. P., Torres, G., et al. 2002, AJ, 124, 1144

Lind, K., Asplund, M., \& Barklem, P. S. 2009a, A\&A, 503, 541

Lind, K., Primas, F., Charbonnel, C., Grundahl, F., \& Asplund, M. 2009b, A\&A, 503,545

Ludwig, H.-G., Behara, N. T., Steffen, M., \& Bonifacio, P. 2009a, A\&A, 502, L1 Ludwig, H.-G., Caffau, E., Steffen, M., et al. 2009b, Mem. Soc. Astron. Ital., 80, 711

Meléndez, J., \& Ramírez, I. 2004, ApJ, 615, L33

Michaud, G., Fontaine, G., \& Beaudet, G. 1984, ApJ, 282, 206

Molaro, P. 2008, Pathways Through an Eclectic Universe, 390, 472

Pettini, M., Zych, B. J., Murphy, M. T., Lewis, A., \& Steidel, C. C. 2008, MNRAS, 391, 1499

Piau, L. 2008, ApJ, 689, 1279

Piau, L., Beers, T. C., Balsara, D. S., et al. 2006, ApJ, 653, 300

Plez, B., Brett, J. M., \& Nordlund, A. 1992, A\&A, 256, 551

Press, W. H., Teukolsky, S. A., Vetterling, W. T., \& Flannery, B. P. 1992 (Cambridge: University Press), 2nd ed.

Richard, O., Michaud, G., \& Richer, J. 2005, ApJ, 619, 538

Ryan, S. G., Beers, T. C., Deliyannis, C. P., \& Thorburn, J. A. 1996, ApJ, 458, 543

Ryan, S. G., Norris, J. E., \& Beers, T. C. 1999, ApJ, 523, 654

Sbordone, L. 2005, Mem. Soc. Astron. Ital. Suppl., 8, 61

Sbordone, L., Bonifacio, P., Castelli, F., \& Kurucz, R. L. 2004, Mem. Soc. Astron. Ital. Suppl., 5, 93

Schlegel, D. J., Finkbeiner, D. P., \& Davis, M. 1998, ApJ, 500, 525

Seaton, M.J. 1962, in Atomic and Molecular Processes, ed. D.R. Bates, 375

Skrutskie, M. F., Cutri, R. M., Stiening, R., et al. 2006, AJ, 131, 1163

Spite, M., \& Spite, F. 1982a, Nature, 297, 483

Spite, F., \& Spite, M. 1982b, A\&A, 115, 357

Stehlé, C., \& Hutcheon, R. 1999, A\&AS, 140, 93

Steigman, G. 2001, [arXiv: astro-ph/0107222]

Steigman, G. 2007, Ann. Rev. Nucl. Particle Science, 57, 463

Steigman, G. 2009, AIPC, 1135, 94

Tolstoy, E., Hill, V., \& Tosi, M. 2009, ARA\&A, 47, 371

Van Regemorter, H. 1962, ApJ, 136, 906

van’t Veer-Menneret, C., \& Megessier, C. 1996, A\&A, 309, 879

Venn, K. A., \& Lambert, D. L. 2008, ApJ, 677, 572

Wagoner, R. V., Fowler, W. A., \& Hoyle, F. 1967, ApJ, 148, 3

Wedemeyer, S., Freytag, B., Steffen, M., Ludwig, H.-G., \& Holweger, H. 2004, A\&A, 414, 1121

Pages 20 to 22 are available in the electronic edition of the journal at http: //www . aanda . org 


\section{Appendix A: 3D non-LTE treatment of lithium}

The calculation of synthetic non-LTE Li I $\lambda 670.8 \mathrm{~nm}$ line profiles from 3D hydrodynamical model atmospheres proceeds in two basic steps. In the first step, the code NLTE3D provides the departure coefficients $b_{i}(x, y, z, t)=n_{i}(x, y, z, t) / n_{i}^{*}(x, y, z, t)$, the ratio of non-LTE to LTE population number densities for each level $i$ of the Li I model atom as a function of the geometrical position $(x, y, z)$ in the $3 \mathrm{D}$ model atmosphere, and time $(t)$ as sampled by a number of snapshots $(\approx 20)$ selected to represent the characteristic temporal variation of the simulation. The departure coefficient of $\mathrm{Li}$ II is assumed to be 1, since lithium is essentially fully ionized in the stellar atmospheres of interest.

In the second step, the departure coefficients are fed into the completely independent spectrum synthesis code Linfor $3 \mathrm{D}^{9}$, where they are used to compute the non-LTE line opacity and source function, and in turn the emergent intensity profiles as a function of $(x, y, \theta, \phi, t)$, where the angles $\theta$ and $\phi$ specify the orientation of the line-of-sight in polar spherical coordinates. Finally, the emergent mean flux profile is obtained by horizontal, angular, and temporal averaging of the individual intensity profiles.

For this paper, we used an 8-level model atom of Li I to solve the statistical equilibrium equations, considering a total of 11 bound-bound transitions. Details about the energy levels and line transitions are given in Tables A.1 and A.2. The Einstein coefficients $A_{j i}$ provided by the NIST database are related to the Einstein coefficients $B_{i j}$ by

$A_{j i}=\frac{2 h v_{i j}^{3}}{c^{2}} B_{j i}=\frac{2 h v_{i j}^{3}}{c^{2}} \frac{g_{i}}{g_{j}} B_{i j}$.

The corresponding oscillator strength $f_{i j}$ is obtained from the relation

$f_{i j}=A_{j i} \frac{g_{j}}{g_{i}} \frac{m_{\mathrm{e}} c^{3}}{8 \pi^{2} e^{2} v_{i j}^{2}}=B_{i j} \frac{m_{\mathrm{e}} h c v_{i j}}{4 \pi^{2} e^{2}}$.

The computationally most expensive part of solving the nonLTE problem is the calculation of the line-blanketed radiation field $J_{v}(x, y, z, t)$ at each grid point of the selected 3D models, which is needed to determine the photoionization rates for all atomic levels. This is done with a modified version of the radiation transport routines that are used in the $C 0^{5} \mathrm{BOLD}$ hydrodynamical simulations for computing the radiative energy exchange term $\nabla \boldsymbol{F}_{\text {rad }}(x, y, z)$. The solution of the radiative transfer equation is based on a Feautrier scheme applied to a set of long characteristics. The continuous opacities used in this context are computed with the routines IONDIS \& OPALAM from the Kiel stellar atmosphere package ${ }^{10}$. Line blanketing is taken into account by adding to the continuous opacity the opacity distribution functions (ODFs, "big division", $v_{\text {turb }}=2 \mathrm{~km} \mathrm{~s}^{-1}$ ) of Castelli \& Kurucz (2003), including the H I- $\mathrm{H}^{+}$and H I-H I quasi-molecular absorption near 1400 and $1600 \AA$, respectively. There is a slight inconsistency in the chemical composition adopted for the calculation of the opacities: the continuous opacities are based on the solar abundances of Asplund et al. (2005), while the ODFs rely on the solar composition of Grevesse \& Sauval (1998). To obtain the opacities for different metallicities, the solar abundances were scaled by a global factor corresponding to the desired $[\mathrm{M} / \mathrm{H}]$, with an enhancement of the $\alpha$-elements

\footnotetext{
9 http://www.aip.de/ mst/Linfor3D/linfor_3D_manual. $\operatorname{pdf}$

${ }^{10}$ http://www .aip.de/ mst/Linfor3D/linfor .pdf
}

Table A.1. Energy levels of the Li I model atom.

\begin{tabular}{ccccc}
\hline \hline $\begin{array}{c}\text { Level } \\
\#\end{array}$ & Configuration & \multicolumn{2}{c}{ Energy } & $\begin{array}{c}\text { Statistical } \\
\text { weight }\end{array}$ \\
\hline 1 & $2 \mathrm{~s}$ & 0.0000000 & 0.00000 & 2 \\
2 & $2 \mathrm{p}$ & 0.1358136 & 1.84784 & 6 \\
3 & $3 \mathrm{~s}$ & 0.2479204 & 3.37313 & 2 \\
4 & $3 \mathrm{p}$ & 0.2818128 & 3.83426 & 6 \\
5 & $3 \mathrm{f}$ & 0.2850726 & 3.87861 & 10 \\
6 & $4 \mathrm{~s}$ & 0.3190534 & 4.34094 & 2 \\
7 & $4 \mathrm{p}$ & 0.3323350 & 4.52165 & 6 \\
8 & $4 \mathrm{~d}$ & 0.3337369 & 4.54072 & 10 \\
\hline
\end{tabular}

Notes. Data are taken from the NIST database.

by 0.4 dex below $[\mathrm{M} / \mathrm{H}]=-0.5$. A total of 600 frequency points were used to obtain the $J_{v}$ between $\lambda 925$ and $19800 \AA$. We checked that treating continuous scattering as true absorption does not introduce any significant changes in the resulting departure coefficients.

Given the line-blanketed radiation field $J_{v}(x, y, z, t)$, the photoionization rate $P_{i \kappa}$ from level $i$ to the continuum $\kappa$ is computed as

$P_{i \kappa}=4 \pi \int_{v_{i}}^{\infty} \frac{\alpha_{i}(v) J_{v}}{h v} \mathrm{~d} v \quad\left[\mathrm{~s}^{-1}\right]$,

where $v_{i}$ is the threshold photoionization frequency for level $i$, and the photoionization cross-sections $\alpha_{i}(v)$ for all considered atomic levels are taken from the TOPBASE Opacity Project online atomic database at the "Centre de données astronomiques de Strasbourg"11. The photo-recombination rates $P_{K i}$ from the continuum $\kappa$ to level $i$ are then given by

$$
\begin{aligned}
P_{\kappa i}= & 4 \pi \int_{v_{i}}^{\infty} \frac{\alpha_{i}(v) B_{v}(T)}{h v}\left(1-\exp \left\{-\frac{h v}{k T}\right\}\right) \mathrm{d} v \\
& +4 \pi \int_{v_{i}}^{\infty} \frac{\alpha_{i}(v) J_{v}}{h v} \exp \left\{-\frac{h v}{k T}\right\} \mathrm{d} v \quad\left[\mathrm{~s}^{-1}\right],
\end{aligned}
$$

$B_{v}(T)$ denoting the Kirchhoff-Planck function at local temperature $T$ and frequency $v$.

Cross-sections for the collisional ionization and excitation by electrons are computed according to the prescriptions of Seaton (1962) and Van Regemorter (1962), respectively, as given by Allen (1976) and Cox (2000). The oscillator strengths $f_{i j}$ from Table A.2 are needed to calculate the collisional excitation cross-sections. Collisional ionization by neutral hydrogen via the charge transfer reaction $\mathrm{H}(1 \mathrm{~s})+\mathrm{Li}(n l) \rightarrow \mathrm{Li}^{+}\left(1 \mathrm{~s}^{2}\right)+\mathrm{H}^{-}$, and the reverse process $\mathrm{H}^{-}+\mathrm{Li}^{+}\left(1 \mathrm{~s}^{2}\right) \rightarrow \mathrm{H}(1 \mathrm{~s})+\mathrm{Li}(n l)$, are treated according to Barklem et al. (2003) for the first 7 levels. Collisional excitation by neutral hydrogen is ignored, as it was found to be unimportant for thermalizing Li I by Barklem et al. (2003).

The departure coefficients $b_{i}(x, y, z)$ are finally obtained by solving the statistical equilibrium equations locally at each grid point $(x, y, z)$. The profile-averaged radiation field at the line transitions, $\bar{J}\left(v_{i j}\right)$, determines the radiative excitation rates

$R_{i j}=B_{i j} \bar{J}\left(v_{i j}\right) \equiv B_{i j} \int_{\text {line } i j} \phi_{v} J_{v} \mathrm{~d} v \quad\left[\mathrm{~s}^{-1}\right]$,

and the radiative de-excitation rates

$R_{j i}=\frac{g_{j}}{g_{i}} B_{j i}\left(\frac{2 h v_{i j}^{3}}{c^{2}}+\bar{J}\left(v_{i j}\right)\right) \quad\left[\mathrm{s}^{-1}\right]$,

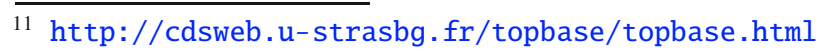


Table A.2. Bound-bound transitions of the Li I model atom.

\begin{tabular}{cccccccc}
\hline \hline $\begin{array}{c}\text { Transition } \\
\#\end{array}$ & $\begin{array}{c}\text { Lower } \\
\text { level }\end{array}$ & $\begin{array}{c}\text { Upper } \\
\text { level }\end{array}$ & $\begin{array}{c}\text { Transition } \\
\text { configuration }\end{array}$ & $\begin{array}{c}\lambda \\
\text { vacuum }[\AA]\end{array}$ & \multicolumn{3}{c}{ Transition probability } \\
$A_{j i}$ & $B_{i j}$ & $f_{i j}$ \\
\hline 1 & 1 & 2 & $2 \mathrm{~s}-2 \mathrm{p}$ & 6709.7 & $3.72 \mathrm{E}+07$ & $8.49 \mathrm{E}+10$ & $7.532 \mathrm{E}-01$ \\
2 & 1 & 4 & $2 \mathrm{~s}-3 \mathrm{p}$ & 3233.6 & $1.17 \mathrm{E}+06$ & $2.99 \mathrm{E}+08$ & $5.502 \mathrm{E}-03$ \\
3 & 1 & 7 & $2 \mathrm{~s}-4 \mathrm{p}$ & 2742.0 & $1.42 \mathrm{E}+06$ & $2.21 \mathrm{E}+08$ & $4.802 \mathrm{E}-03$ \\
4 & 2 & 3 & $2 \mathrm{p}-3 \mathrm{~s}$ & 8128.6 & $1.74 \mathrm{E}+07$ & $7.84 \mathrm{E}+09$ & $5.745 \mathrm{E}-02$ \\
5 & 2 & 5 & $2 \mathrm{p}-3 \mathrm{~d}$ & 6105.3 & $5.11 \mathrm{E}+07$ & $4.88 \mathrm{E}+10$ & $4.759 \mathrm{E}-01$ \\
6 & 2 & 6 & $2 \mathrm{p}-4 \mathrm{~s}$ & 4973.1 & $5.05 \mathrm{E}+06$ & $5.21 \mathrm{E}+08$ & $6.241 \mathrm{E}-03$ \\
7 & 2 & 8 & $2 \mathrm{p}-4 \mathrm{~d}$ & 4604.1 & $1.64 \mathrm{E}+07$ & $6.71 \mathrm{E}+09$ & $8.687 \mathrm{E}-02$ \\
8 & 3 & 4 & $3 \mathrm{~s}-3 \mathrm{p}$ & 26887.1 & $3.77 \mathrm{E}+06$ & $5.53 \mathrm{E}+11$ & $1.226 \mathrm{E}+00$ \\
9 & 3 & 7 & $3 \mathrm{~s}-4 \mathrm{p}$ & 10795.1 & $3.69 \mathrm{E}+03$ & $3.51 \mathrm{E}+07$ & $1.934 \mathrm{E}-04$ \\
10 & 4 & 8 & $3 \mathrm{p}-4 \mathrm{~d}$ & 17550.0 & $4.89 \mathrm{E}+06$ & $1.11 \mathrm{E}+11$ & $3.763 \mathrm{E}-01$ \\
11 & 5 & 7 & $3 \mathrm{~d}-4 \mathrm{p}$ & 19281.0 & $3.31 \mathrm{E}+05$ & $3.58 \mathrm{E}+09$ & $1.107 \mathrm{E}-02$ \\
\hline
\end{tabular}

Notes. Data are taken from the NIST database.

where the statistical weights of lower and upper level, $g_{i}$ and $g_{j}$, and the Einstein coefficients $B_{i j}$ are taken from Table A.2. For the line profile $\phi_{v}$ we assume a purely Gaussian distribution (identical for absorption and emission) with a line width that corresponds to the local thermal Doppler velocity plus a microturbulence of $1.5 \mathrm{~km} \mathrm{~s}^{-1}$. The Doppler shift caused by the line-ofsight component of the hydrodynamical velocity field is ignored in the present computation. For all transitions except for the resonance line, $\bar{J}\left(v_{i j}\right)$ was replaced by the mean continuum intensity $J_{v}$. For the resonance line (transition \#1), the line opacity was taken into account for the computation of $\bar{J}\left(v_{i j}\right)$, assuming an $f$-value of 0.48975 (about $2 / 3$ of the value given in Table A.2 to represent only the main component of the doublet), together with a typical lithium abundance of $A(\mathrm{Li})=2.2$.

Since $\bar{J}\left(v_{i j}\right)$ is a non-local quantity that depends in turn on the $b_{i}$, a $\Lambda$-iteration is employed to obtain a consistent solution. Fortunately, the $\Lambda$ iteration converges very rapidly, because even the Li I resonance line affects the radiation field only marginally, while all the other lines are very weak. Typically, three iterations are sufficient to achieve convergence.

Finally, the departure coefficients $b_{i}$ are used in the line formation code Linfor 3D to compute the non-LTE line opacity

$\kappa_{\text {line }}($ non - LTE $)=\frac{b_{\text {low }}-b_{\text {up }} \exp \left\{-\frac{h v}{k T}\right\}}{1-\exp \left\{-\frac{h v}{k T}\right\}} \kappa_{\text {line }}($ LTE $)$,

and the line source function

$S_{v, \text { line }}($ non - LTE $)=b_{\text {up }} \frac{1-\exp \left\{-\frac{h v}{k T}\right\}}{b_{\text {low }}-b_{\text {up }} \exp \left\{-\frac{h v}{k T}\right\}} B_{v}$,

where $b_{\text {low }}$ and $b_{\text {up }}$ are the departure coefficients of lower and upper level, respectively, and $B_{v}$ is again the Kirchhoff-Planck function.

\section{Appendix B: Analytical fit to 3D NLTE Li abundance as a function of stellar parameters and $E W$}

\section{B.1. Introduction}

It is often convenient to have tabular data on an irregular grid $y=y(x)$ condensed into a single function (even with many fit parameters), instead of having to interpolate between the original data points. Choosing a fitting function that is linear in all parameters (e.g., a sum of polynomials as $A+B x+C x^{2}$ or sine/cosine functions) results in a comparatively simple linear problem for finding these parameters, usually with one unique solution. However, the result might not have the desired extrapolation behavior, might be "too wiggly" between the data points, or produce no good fit at all.

A more effective approach might then be to start with a properly crafted function, possibly non-linear, and having fitting parameters "within" the terms as in $A \exp (B+C x)$. In this case, an iterative process is necessary to determine these parameters. This iteration requires starting values, which will affect the convergence of the process and also the solution if one is found: in this case, the scheme might diverge or converge to different local minima, depending on the starting point. However, this last issue is hardly a problem for our application - we are content with a good fit, and do not necessarily need to achieve the closest one. We continue, however, to have the problem of constructing the fitting function and choosing the initial values for the parameters.

\section{B.2. Method}

We used a scheme for recursive term substitution, written in IDL, that attempted not only to find optimum fitting parameters for a given function, but also to automatically determine the optimum functional form itself. The latter was chosen from a set of candidates given by a list of terms and some construction rules. The usual starting point was a constant "function" with one parameter, say $A$, the best "fit" for which is the mean of $y$. In each recursion step, all parameters were successively substituted with all of the terms from a list (with possible restrictions), for instance

$A \rightarrow A_{0}+A_{1} x$

or

$A \rightarrow A_{0}+A_{1} \exp \left(A_{2} x\right)$.

The initial values of $\left(A_{0}, A_{1}, A_{2}\right)$ were derived from $A$ by e.g., assuming $A_{0}=A, A_{1}=0.1 \times A, A_{2}=0$. Applying a modified version of the IDL function curvefit (requiring also partial derivatives) provided the optimum parameter set for the given function and start values.

This process was repeated with a number of random variations to approach an optimum solution for the given function. The final solution was the globally best fit among all candidate functions and parameters. 
The main control parameters are the list of candidate terms and the recursion depth. For the current application, we search a 4D function

$y=y\left(x_{0}, x_{1}, x_{2}, x_{3}\right)$

where $\left(x_{0}, x_{1}, x_{2}, x_{3}\right)=\left(\log (E W), \log T_{\text {eff }}, \log g\right.$, $\left.[\mathrm{Fe} / \mathrm{H}]\right)$, or, for the inverse problem, $\left(A(\mathrm{Li}), \log T_{\text {eff }}, \log g,[\mathrm{Fe} / \mathrm{H}]\right)$. We start with

$A_{0}+\log _{10}\left[1-\exp \left(-10^{\left(X_{0}-A_{1}\right)}\right)\right]$

to determine the equivalent width as a function of abundance, based on a simple absorption model for a box-shaped line profile. In the following recursion steps, we replace the parameters with linear terms

$A \rightarrow A_{0}+A_{1} * x_{1}+A_{2} x_{2}+A_{3} x_{3}$,

which finally results in higher-order polynomials instead of the initial coefficients. In this step, the variation in the COG with the stellar parameters is taken into account. In the process of applying the term substitution, we scanned through several hundred possible functional forms. We decided the most suitable one on the basis of simplicity, and its ability to represent the numerical data with sufficient accuracy.

As described in Sect. 6.1, synthetic grids were computed for three cases, 3D NLTE, 1D LHD NLTE, and 1D LHD LTE. This in principle leads to 6 fitting functions being computed, when both the $E W \rightarrow A(\mathrm{Li})$ and $A(\mathrm{Li}) \rightarrow E W$ forms are required. We decided to force all three functions in either sense to have the same form, differing only by their parameters. We thus first searched an optimum function for one case $(A(\mathrm{Li}) \rightarrow 1 \mathrm{D}$ LHD NLTE turned out to be the optimum choice) and then varied only the parameters (and not the functional form) for the other two cases. The final inversion of the function was performed by hand.

\section{B.3. Best-fitting functions}

Best-fitting functional forms were produced both to derive $A(\mathrm{Li})$ from $E W$ and to derive $E W$ from $A(\mathrm{Li})$, the latter being useful, e.g., for the preparation of observations. Analytical fit formulae for both cases are presented in Eq. (B.1); fitting coefficients are listed in Table B.1. Three sets of coefficients are listed. The $3 \mathrm{D}$ NLTE set is used to fit computations where CO ${ }^{5}$ BOLD 3D hydrodynamical models are used in association with a 3D NLTE time-dependent spectrosynthesis. The 1D NLTE and LTE cases refer instead to computations performed by using LHD onedimensional models, with spectrosynthesis performed with or
Table B.1. Coefficients for the analytical fit of $E W \rightarrow A(\mathrm{Li})$ and $A(\mathrm{Li}) \rightarrow E W$.

\begin{tabular}{cccr}
\hline \hline & 3D NLTE & 1D NLTE & \multicolumn{1}{c}{ 1D LTE } \\
\hline$A_{0}$ & $2.1744416 \mathrm{E}+00$ & $2.2011840 \mathrm{E}+00$ & $2.1694977 \mathrm{E}+00$ \\
$A_{1}$ & $3.9685178 \mathrm{E}+02$ & $-2.3708574 \mathrm{E}+02$ & $-3.1329205 \mathrm{E}+02$ \\
$A_{2}$ & $-2.1920459 \mathrm{E}+02$ & $9.9754471 \mathrm{E}+01$ & $1.5692142 \mathrm{E}+02$ \\
$A_{3}$ & $-9.8448749 \mathrm{E}+02$ & $1.5211893 \mathrm{E}+02$ & $1.9027467 \mathrm{E}+03$ \\
$A_{4}$ & $2.4222436 \mathrm{E}+00$ & $-7.0910416 \mathrm{E}+00$ & $8.0236683 \mathrm{E}+00$ \\
$A_{5}$ & $3.0470810 \mathrm{E}+01$ & $-9.5952034 \mathrm{E}+00$ & $-1.9364346 \mathrm{E}+01$ \\
$A_{6}$ & $7.4822784 \mathrm{E}+02$ & $-1.0479124 \mathrm{E}+02$ & $-1.4933164 \mathrm{E}+03$ \\
$A_{7}$ & $-6.0743892 \mathrm{E}-01$ & $1.8554645 \mathrm{E}+00$ & $-2.1075335 \mathrm{E}+00$ \\
$A_{8}$ & $-1.8920995 \mathrm{E}+02$ & $2.5357561 \mathrm{E}+01$ & $3.9087711 \mathrm{E}+02$ \\
$A_{9}$ & $-7.9977289 \mathrm{E}-02$ & $-7.5381881 \mathrm{E}-01$ & $-2.7466467 \mathrm{E}-01$ \\
$A_{10}$ & $-3.2394665 \mathrm{E}-01$ & $3.8146901 \mathrm{E}-01$ & $-4.9764225 \mathrm{E}-01$ \\
$A_{11}$ & $1.5911137 \mathrm{E}+01$ & $-2.1918788 \mathrm{E}+00$ & $-3.4126404 \mathrm{E}+01$ \\
$A_{12}$ & $2.3078753 \mathrm{E}-02$ & $1.9988796 \mathrm{E}-01$ & $7.3497586 \mathrm{E}-02$ \\
$A_{13}$ & $8.3344564 \mathrm{E}-02$ & $-9.9645615 \mathrm{E}-02$ & $1.3085939 \mathrm{E}-01$ \\
\hline
\end{tabular}

without the inclusion of NLTE effects, based on the same physics and model atoms as for the 3D NLTE case:

$Q_{1}=A_{8}+A_{11} \log T_{\text {eff }}+A_{12} \log g+A_{13}[\mathrm{Fe} / \mathrm{H}]$

$Q_{2}=A_{6}+Q_{1} \log T_{\text {eff }}+A_{9} \log g+A_{10}[\mathrm{Fe} / \mathrm{H}]$

$Q_{3}=A_{2}+A_{5} \log T_{\text {eff }}+Q_{2} \log g+A_{7}[\mathrm{Fe} / \mathrm{H}]$

$Q_{4}=A_{1}+Q_{3} \log T_{\text {eff }}+A_{3} \log g+A_{4}[\mathrm{Fe} / \mathrm{H}]$

$A(\mathrm{Li})=A_{1}+Q_{3} \log T_{\text {eff }}+A_{3} \log g+A_{4}[\mathrm{Fe} / \mathrm{H}]$

$+\log \left[-\ln \left(1-E W 10^{-A_{0}}\right)\right]$

$\log E W=A_{0}+\log \left[\left|1+\exp \left(-10^{A(L i)-Q_{4}}\right)\right|\right]$.

Units are expected to be $\mathrm{K}$ for temperature, $\mathrm{cm} \mathrm{s}^{-2}$ for $\log g$, and $\mathrm{m} \AA$ for $E W$. As for any fit, extrapolation reliability is difficult to assess. Owing to the high computational cost of 3D-atmosphere model calculations, we limited our grid to the currently available models, which obliged us to extrapolate towards high gravities by 0.4 dex and towards low metallicities by about 0.6 dex. Both extrapolations should be quite safe, since the $670.8 \mathrm{~nm} \mathrm{Li}$ doublet should be quite insensitive to both parameters. Because of the vanishing line opacity, atmosphere models are scarcely sensitive to metallicity variations below $[\mathrm{Fe} / \mathrm{H}]=-3$. Those who might wish to employ the presented formulae to derive Li abundances are nevertheless advised to use caution when extrapolating, especially in $T_{\text {eff }}$ and $E W$. In particular, the saturation part of the COG is almost unsampled, and one cannot expect the present fit to reproduce it properly. Fitting to $E W$ significantly above $100 \mathrm{pm}$ is therefore not advisable. 